\title{
CONFORMAL INVARIANCE IN TWO-DIMENSIONAL PERCOLATION
}

\author{
ROBERT LANGLANDS, PHILIPPE POULIOT, AND YVAN SAINT-AUBIN
}

\section{CONTENTS}

1. Introduction.

2. The hypotheses of universality and conformal invariance.

2.1 Basic results and questions in percolation.

2.2 Universality and the renormalization group.

2.3 Crossing probabilities.

2.4 The two hypotheses.

2.5 More critical indices for percolation.

2.6 Conformally invariant fields and percolation.

3. The experiments.

3.1 Experimental procedure.

3.2 Experimental verification of Cardy's formula.

3.3 Parallelograms.

3.4 Striated models.

3.5 Exterior domains.

3.6 Branched percolation.

3.7 Percolation on compact Riemann surfaces.

\section{INTRODUCTION}

The word percolation, borrowed from the Latin, refers to the seeping or oozing of a liquid through a porous medium, usually to be strained. In this and related senses it has been in use since the seventeenth century. It was introduced more recently into mathematics by S. R. Broadbent and J. M. Hammersley $([\mathrm{BH}])$ and is a branch of probability theory that is especially close to statistical mechanics. Broadbent and Hammersley distinguish between two types of spreading of a fluid through a medium, or between two aspects of the probabilistic models of such processes: diffusion processes, in which the random mechanism is ascribed to the fluid; and percolation processes, in which it is ascribed to the medium.

A percolation process typically depends on one or more probabilistic parameters. For example, if molecules of a gas are absorbed at the surface of a porous solid (as in a gas mask) then their ability to penetrate the solid depends on the

Received by the editors October 4, 1992 and, in revised form, December 8, 1992, and July 20, 1993.

1991 Mathematics Subject Classification. Primary 82B43; Secondary 82B27.

Key words and phrases. Percolation, conformal invariance, critical phenomena, conformal quantum field theory.

A first version of part of the material of this paper was presented by the first author as part of the AMS Colloquium lectures in Baltimore in January 1992.

The third author was supported in part by NSERC Canada and the Fonds FCAR pour l'aide et le soutien à la recherche (Québec). 
sizes of the pores in it and their positions, both conceived to be distributed in some random manner. A simple mathematical model of such a process is often defined by taking the pores to be distributed in some regular manner (that could be determined by a periodic graph), and to be open (thus very large) or closed (thus smaller than the molecules) with probabilities $p$ and $1-p$. As $p$ increases the probability of deeper penetration of the gas into the interior of the solid grows.

There is often a critical threshold for the probability at which the behavior changes abruptly - below which the penetration is only superficial, and above which it is infinitely deep. Such critical behavior is a very simple analogue of similar behavior in thermodynamics and statistical mechanics that is of great theoretical and experimental, as well as mathematical, interest. Since the critical behavior manifested in percolation shares many characteristics with that of more complex systems and models, percolation has attracted wide interest $([\mathrm{G}, \mathrm{K}])$ among physicists and mathematicians as one of the simplest cases in which various striking features of critical behavior, especially scaling and universality, appear. These two terms are central to this paper, and will be discussed more at length below. Scaling refers, in essence, to the frequent appearance of simple power laws. The exponent in these laws is often the same for quite different materials and models, and this is called universality.

The immediate purpose of the paper was neither to review the basic definitions of percolation theory nor to rehearse the general physical notions of universality and renormalization (an important technique to be described in Part Two). It was rather to describe as concretely as possible, although in hypothetical form, the geometric aspects of universality, especially conformal invariance, in the context of percolation, and to present the numerical results that support the hypotheses. On the other hand, one ulterior purpose is to draw the attention of mathematicians to the mathematical problems posed by the physical notions. Some precise basic definitions are necessary simply to orient the reader. Moreover a brief description of scaling and universality on the one hand and of renormalization on the other is also essential in order to establish their physical importance and to clarify their mathematical content.

These matters are all treated in Part Two. Since one of its purposes is to orient ourselves and other inexperienced mathematicians with respect to the physical background, we have not shrunk from the occasional doubtful utterance that shed, for us at least, some light in an obscure corner. We urge the reader to be especially circumspect while reading $\S 2.2$. That we are dealing there with material with which none of us has had first-hand experience is not the least of the reasons, but it is also not the only one.

The first paragraph of Part Two is deliberately stark. We hope that the content of the questions posed there is clear; their depth cannot be at this stage. They are central and inaccessible, but as problems they are the source of the hypotheses of $\$ 2.4$ and the experiments described in Part Three.

The attention given to $\$ 2.2$ will depend on the reader's familiarity with the physical concepts used. Many are fairly close to everyday experience, but there are also deep ideas with a long history compressed into single phrases. Fortunately the section can be skipped completely, and those with no experience with the concepts can pass directly, or at least quickly, to $\S 2.3$ and $\S 2.4$ which are prerequisites to Part Three. $\S 2.2$ is not. Nor are the final two paragraphs of Part 
Two. $\S 2.5$ is an appendix, in the context of percolation, to $\S 2.2$. The material in $\S 2.6$ is especially difficult, but especially important because it illustrates the power of the methods of conformal field theory for making analytic predictions. These appear to be far less accessible to rigorous mathematical demonstration, and perhaps deeper, than more familiar geometric predictions. The ideas of $\S 2.6$ are due to Cardy, and appear in a sequence of papers. In spite of their lack of rigor, they appear to be of great potential, and our purpose is simply to present them in the most accessible form we could manage.

Since only the statements of the hypotheses are strict prerequisites for it, Part Three, far more elementary than Part Two, can be read without a thorough understanding of the preceding part. By the same token, Part Three can be taken as nothing more than an illustration of what happens when mathematicians take the physical ideas of Part Two seriously, and Part Two can be read without reference to it.

After the discussion of the general experimental procedure in $\S 3.1$, the description of the experiments begins. It is, of course, the experiments that give substance to the paper, in which nothing is proved mathematically. $\S 3.2$ offers a table of approximate, but statistically very precise results obtained by simulation that serve two purposes: a verification with better data than those of [U] of the formula of Cardy in $\S 2.6$; construction of a collection of data with which the less precise data of the following sections may be compared.

The numerical investigation of conformal invariance is begun in $\S 3.3$. The data of $\S 3.2$ are for rectangles. The interior of every parallelogram is conformally equivalent to the interior of an appropriate rectangle, and the conformal mapping is uniquely determined if it is insisted that vertices be taken to vertices. Moreover the aspect ratio $r$ of the rectangle (the quotient of the lengths of neighboring sides) is all but uniquely determined. The only possibility is that $r$ be replaced by $1 / r$. Thus a natural first comparison to establish conformal invariance is to compare data for parallelograms with the standard data of $\S 3.2$ for rectangles. This is done in $\S 3.3$.

The notion of universality of $\S 2.4$ is not that of $\S 2.2$, but closely related to it; and as remarked in $[\mathrm{U}]$ it is difficult to determine to what extent it was accepted in the community of specialists. It has certainly not been exploited. Specialists are not inclined to doubt it when questioned closely and it has been tested in a restricted form in [U]. In $\S 3.4$, we content ourselves with a single example of the general hypothesis, whose purpose is principally to exhibit an example in which all symmetries are violated, and to show how to make calculations for it.

The final three sections in Part Three are a more adventurous pursuit of the consequences of conformal invariance of percolation. We define percolation on a variety of Riemann surfaces: unbounded planar domains; branched coverings of bounded planar domains; and then on branched coverings of the Riemann sphere. We stop there, but we could have gone farther. The principle has certainly become clear. In each case, we take an example and verify conformal invariance for it, but for reasons that we explain the precision with which we verify this invariance decreases. Thus the numerical evidence for conformal invariance in the generality it is finally conceived is not so good as it could be with more painstaking experiments, but even those performed took considerable time, and provide evidence that is positive, and in our view convincing. Our aim was less to achieve great precision than to assure ourselves that even bold 
forms of the hypothesis of conformal invariance stood a good chance of being valid. Although further precision is certainly desirable, it seems to us that the search for proofs can begin with some moral certainty that the general assertions implicit (the reader will have no difficulty in making them explicit) in the last three sections are valid.

As far as we have been able to determine (with the help provided by A. Mortensen of the Department of Material Sciences at MIT) the study of critical behavior and universality in percolation is of much less practical than theoretical importance. The paper [M] of MacLachlan et al and that of Wong [W] suggest that in such practical applications of percolation processes as the study of composite materials or the porosity of rocks the interest is less in quantities similar to that of the theorem of $\S 2.1$ that change abruptly at the critical threshold than in quantities such as conductivity or permeability that change continuously, although with an infinite derivative, across this threshold. The critical indices of this paper are important in so far as they influence the equations governing these quantities, but the principal practical problem is perhaps to reduce, geometrically or otherwise, the critical threshold, for this means incorporating less of a perhaps expensive additive in an inexpensive matrix. Our concerns are theoretical and mathematical.

\section{THE HYPOTHESES OF UNIVERSALITY AND CONFORMAL INVARIANCE}

2.1. Basic results and questions in percolation. A standard model of percolation is that attached to sites on a square lattice. Let $L$ be the graph (embedded in $\mathbb{R}^{d}$ ) whose set of vertices or sites is the set of integral points $\mathbb{Z}^{d}$ and whose edges or bonds join all pairs of nearest neighbors. Each site can be in one of two states. It can be open and then we assign it the value 1 , or it can be closed, and be assigned the value 0 . A configuration is obtained by specifying which sites are open and which are closed. Clearly the set $X$ of all configurations is

$$
\prod_{\mathbb{Z}^{d}}\{0,1\},
$$

the set of functions from $\mathbb{Z}^{d}$ to $\{0,1\}$. A site $s$ is open for a configuration if the corresponding function takes the value 1 at $s$. If $0 \leq p \leq 1$ then we associate to $p$ the probability on $\{0,1\}$ that assigns the probability $p$ to 1 , and introduce the product of these probabilities on the set of all configurations. Each site can then be regarded as an independent random variable assuming two possible values 0 or 1 . We refer to the set $X$ with this probability measure as the model $M_{0}$ of percolation.

For many purposes it is convenient to work not with the full graph $L$ but with the sites

$$
\{(i, j) \mid 1 \leq i, j \leq n\}
$$

in a square $S_{n}$ of side $n$ and the bonds connecting them. If

$$
X_{n}=\prod_{S_{n}}\{0,1\},
$$

then configurations $x \in X_{n}$ are determined by fixing a state for each site in $S_{n}$. The probability $\pi(x)$ of $x$ is equal to $p^{k}(1-p)^{l}$ if $k$ sites are open for $x$, and $l=n^{2}-k$ are closed. A typical configuration $x$ is shown in Figure 2.1a, in which open sites appear as black dots and closed sites are white. 


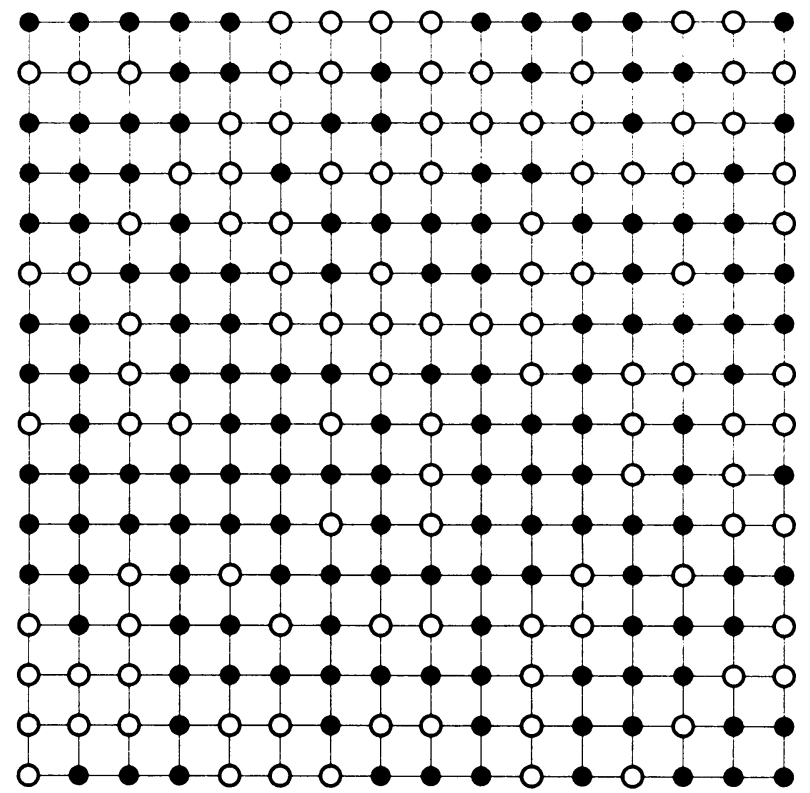

Figure 2.1a. Configurations on the square cube $S_{16}$ for percolation by sites.

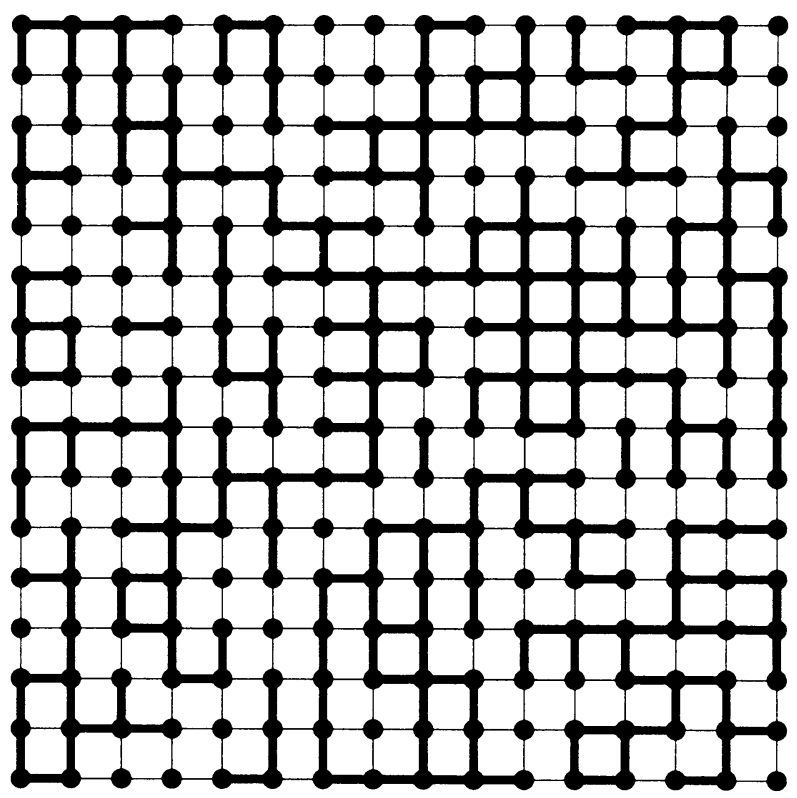

FIGURE 2.1b. Configurations on the square cube $S_{16}$ for percolation by bonds. Both (a) and (b) have a horizontal crossing but no vertical one.

There are many different events in $X$ or $X_{n}$ whose probabilities are of interest in the study of percolation. We shall return to them in $\$ 2.3$. For now, in order to put the questions in stark simplicity, we concentrate on a very special probability $\pi_{h}$, that of a horizontal crossing. Consider the configuration $x$ on 


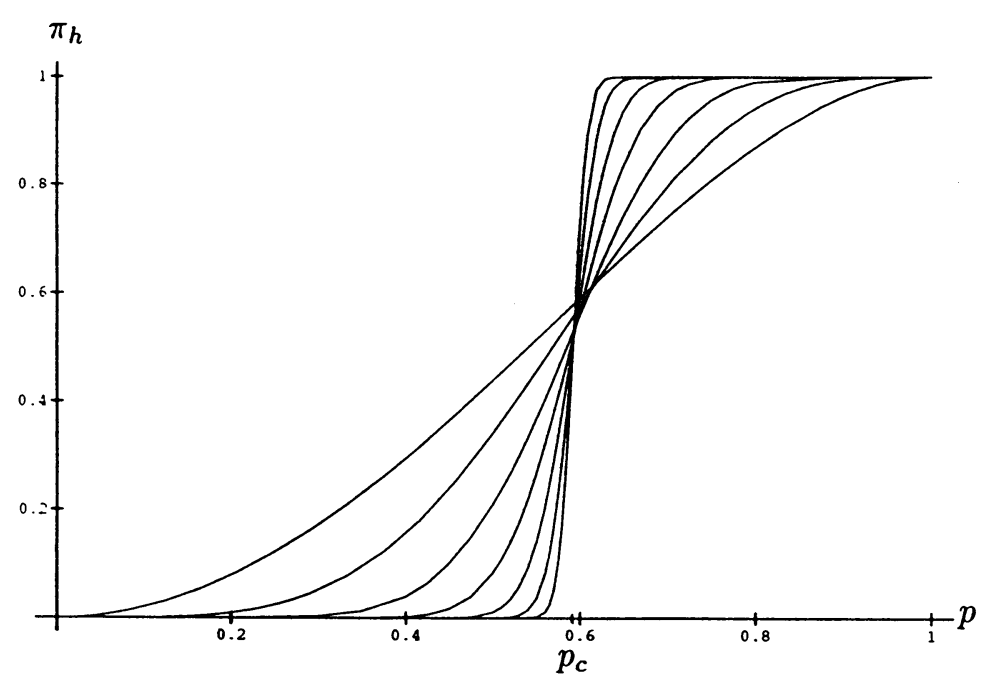

FIGURE 2.1c. The curves $\pi_{h}^{n}(p)$ for $n=2,4,8,16$, 32,64 , and 128. Larger slopes around $p_{c}$ correspond to larger values of $n$.

$S_{16}$ of Figure 2.1a. This configuration admits a horizontal crossing in the sense that it is possible to pass from the left side of the square to the right one by moving repeatedly from one site open for $x$ to another open site joined to it by a bond, thus to an open immediate neighbor. It does not, however, admit a vertical crossing. The probability $\pi_{h}^{n}(p)$ of a horizontal crossing is the sum of the probabilities $\pi(x)$, taken over all configurations $x \in X_{n}$ on $S_{n}$ that admit a horizontal crossing.

The probability $\pi_{h}^{n}(p)$ clearly increases from 0 to 1 as $p$ does. Its behavior with respect to $n$ is revealed by Figure 2.1c, in which the graph of the function $\pi_{h}^{n}$ is given for $n=2,4,8,16,32,64,128$. It appears to be approaching a step function; this is confirmed by the first two statements of the following theorem, whose original proof takes up most of the book [K] of Kesten. A full account of the contributions of earlier authors can be found there. A more recent proof can be found in $[\mathrm{AB}]$.

Theorem. There exists a unique critical probability $0<p_{c}<1$ such that:

(1) for $p<p_{c}$,

$$
\lim _{n \rightarrow \infty} \pi_{h}^{n}(p)=0
$$

(2) for $p>p_{c}$,

$$
\lim _{n \rightarrow \infty} \pi_{h}^{n}(p)=1
$$

(3) for $p=p_{c}$,

$$
0<\liminf _{n \rightarrow \infty} \pi_{h}^{n}(p) \leq \limsup _{n \rightarrow \infty} \pi_{h}^{n}(p)<1 .
$$

In spite of the difficulty and importance of the theorem, it has an obvious defect for it does not answer the question that immediately springs to mind upon reading the final statement. 
Question 1. Does

$$
\lim _{n \rightarrow \infty} \pi_{h}^{n}(p)
$$

exist for $p=p_{c}$ ?

The numerical evidence leaves no doubt that the limit, which we denote $\pi_{h}$, exists. A second question, far more subtle, is also strongly suggested by the numerical data. Consider the derivative $A_{n}$ of $\pi_{h}^{n}(p)$ with respect to $p$ at $p=p_{c}$. If Figure 2.1c does not deceive then $A_{n}$ increases with $n$ and approaches infinity.

Question 2. Does there exist a positive real number $\nu$ such that

$$
\lim _{n \rightarrow \infty} \frac{A_{n}}{n^{\frac{1}{\nu}}}
$$

exists and is different from 0 ?

This is a simple example of a scaling law, a notion that will be explained more generally in the next section.

The two questions, as well as the theorem, have been formulated for the specific model $M_{0}$, but there are many other possible models. For example, in dimension two the lattice $\mathbb{Z}^{2}$ can be replaced by a triangular (or hexagonal) lattice in which each site has 6 (3) nearest neighbors. Percolation by sites can also be replaced with percolation by bonds. In bond percolation all sites are open and it is the bonds that are open with probability $p$. A configuration on $S_{16}$ is shown in Figure 2.1b. The definitions introduced for site percolation on $M_{0}$ are applicable to these new models. The configuration in the figure admits a horizontal crossing but no vertical crossing. One can also study percolation on more general planar graphs, allowing in addition both sites and bonds to be open or closed, and probabilities that depend on the type of bond or site. We could, for example, in bond percolation on a square lattice permit the horizontal and vertical bonds to be open with different probabilities $p_{h}$ and $p_{v}$. The variations are endless, but for all models within a large class, the theorem, in an appropriate form, remains valid, and the questions appear to continue to have an affirmative response. The critical probabilities vary from model to model, but the evidence strongly suggests that yet another, a third, question has an affirmative answer.

Question 3. Is the value $\nu$ independent of the model?

The number $\nu$ is known as a critical index and its independence of the model is known as universality. For reasons not germane to this paper $\nu$ is generally believed to be equal to the rational number $\frac{4}{3}$ for the models of percolation in two dimensions that we study here.

The first, obvious advantage of percolation models is the facility with which $\nu$ can be introduced. In statistical mechanics singular behavior of quantities such as specific heat or magnetic susceptibility is also described by critical indices, to be discussed in the next paragraph, whose constancy within large classes of models, thus their universality, is well established within the limits of experimental observation. Although its sources are not understood, there is a very powerful method, the renormalization group, for analyzing critical behavior, but the problem of understanding the mechanism that allows the geometry to predominate and to efface the details of the interactions and, as a consequence, to render 
renormalization so effective remains. The missing insight can be regarded as physical or mathematical; it is not a question of adding rigor to arguments that are otherwise persuasive. There are none.

The renormalization group was taken, as its name suggests, into statistical mechanics from the theory of quantum fields, and has therefore a conceptually very difficult history with which we are not concerned, although some attempt will be made during the course of the paper to give the phrase some meaning to the reader. It should then be clear to him that, contrary to the first impression, the three questions are not at an ever increasing level of difficulty, so that an earlier one must be answered before a later one can be posed. They must rather be answered simultaneously.

With this in mind, our purpose, in [L1,L2] and [U], has been to introduce objects that deserve to be called renormalizations, but that are at the same time concrete, elementary mathematical objects amenable to rigorous mathematical investigation.

What is introduced in [ $[\mathrm{L} 1, \mathrm{~L} 2]$ is a sequence of continuous transformations of finite-dimensional spaces. They are briefly reviewed in $\$ 2.3$. To relate these objects to renormalization requires hypotheses whose validity was not universally accepted. To assure ourselves that the definitions were well-founded we examined crossing probabilities like $\pi_{h}$ for various models of percolation in [U]. Conversations with Michael Aizenman after the data were in hand greatly clarified for us their nature. In particular he suggested that these crossing probabilities would be conformally invariant.

Subsequent conversations with other mathematicians persuaded us that with the appearance of conformal invariance percolation becomes a topic that appeals to a broader audience than mathematical physicists and probabilists. For example, a remark of Israel Gelfand, for which we are grateful, led to the examination of conformal invariance on compact Riemann surfaces. Since proofs of conformal invariance will likely have to wait upon proofs of universality for percolation, and these, even if the ideas of [L1,L2] have some validity, will in all likelihood be slow in coming, we decided to present the numerical evidence for conformal invariance and its consequences in a form that emphasizes its mathematical appeal, and this is the primary purpose of the present paper. No theorems are proved or implied.

As promised, we preface the numerical results with an explanation, tailored to our concerns, of the terms, universality and renormalization, just invoked. Before beginning, we would like to express our thanks to Michael Aizenman and to Thomas Spencer for their encouragement.

2.2. Universality and the renormalization group. Statistical mechanics and the closely related subject of thermodynamics deal, to some extent, with objects familiar to all of us: gases, liquids, and solids; or magnets in magnetic fields. It comes, therefore, as somewhat of a shock to learn that these substances are not so familiar as we might think. Water vapor, water, and ice and the transitions between them are matters of daily experience, and phase diagrams like Figure $2.2 \mathrm{a}$ frequently met.

They are not usually drawn to scale nor do we ask ourselves which region or values of the pressure and temperature are accessible under normal conditions. Temperatures between $-20^{\circ} \mathrm{C}$ and $100^{\circ} \mathrm{C}$, the boiling point of water, are the 


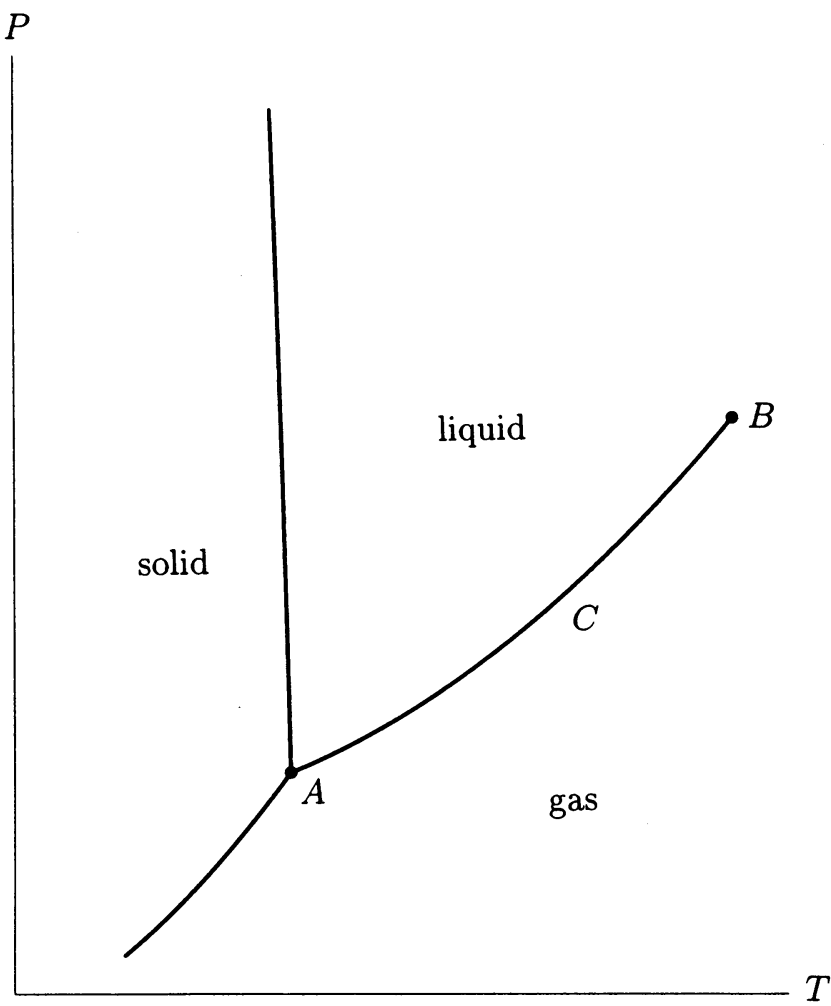

FIGURE 2.2a. Qualitative phase diagram for water.

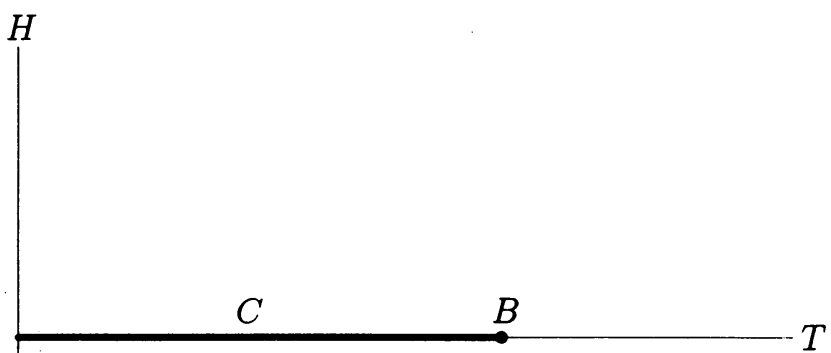

FIGURE 2.2b. Qualitative phase diagram for a ferromagnet.

most common, except under incendiary conditions. Because of the phenomenon of partial pressure, more familiar to us as the numerator in the humidity, only the pressure of the water vapor in the ambient air affects the rate of evaporation 
or thaw, so that the pertinent range of pressures is from 1. atm all the way down to 0 . atm. Thus, even though the triple point $A$ in Figure $2.2 \mathrm{a}$ is at $(P, T)=$ $\left(0.006 \mathrm{~atm}, 0{ }^{\circ} \mathrm{C}\right)$, ice does melt on the surface of ponds and puddles.

On the other hand, the point $B$, the critical point in the technical sense, is at $\left(P_{c}, T_{c}\right)=\left(218\right.$. atm, $\left.341 .{ }^{\circ} \mathrm{C}\right)$, so that no diagram drawn to scale could include the two points. The pressure is that found more than two kilometers under the ocean surface, not a familiar location, and certainly not one in which we might try to boil water.

Thus the phenomena associated with the critical point, and it is for them that universality is pertinent, are not those associated to the transition from water to ice or from water to water vapor. They are of a different nature. If at a fixed temperature $T$ below $T_{c}$ we continuously increase the pressure (or reduce the volume) on a closed container of water vapor then, when the pressure is such that $(P, T)$ lies on the curve $C$, it will start to condense and we will be able to continue to reduce the volume without changing the pressure until there is no vapor left. At this point, continued reduction of the volume will increase the pressure, or more kinesthetically, continued increase of the pressure will reduce the volume, which will have decreased considerably. It is best to imagine the transition occurring in the absence of a gravitational field, so that the difference of density does not cause, in the familiar way, the liquid to precipitate out. Rather a kind of slush is formed during the transition, pockets of liquid in the ambient vapor, or pockets of air in the ambient liquid.

At the point on the curve, where the volume, and therefore the density $\rho$, changes without any change in the pressure, the isothermal compressibility

$$
K_{T}=\frac{1}{\rho}\left(\frac{\partial \rho}{\partial P}\right)_{T}
$$

is of course infinite. Above $T_{c}$ the curve $C$ has terminated and there is no transition from vapor to liquid, rather there is simply a fluid that is gradually becoming denser with the increase in pressure. In particular, at no point does $K_{T}$ become infinite.

If the pressure is increased in the same way at $T=T_{c}$, the behavior can be expected to mimic both that at $T<T_{c}$ and at $T>T_{c}$. The curve given by setting $T$ equal to a constant $T_{c}$ and letting $P$ vary could be replaced by other curves passing through the critical point, but it is better to work with a fixed, simply defined curve. We observe, anticipating a later section, that the critical behavior of percolation, in which there is only one free parameter, the probability, is to be compared with the behavior along such a curve.

The fluid, whether a liquid or a gas, is composed of molecules that are subject to thermal fluctuations, so that the density is only defined for statistically significant aggregates of molecules. Away from the curve $C$ a few molecules suffice (cf $[\mathrm{P}]$ ) so that the normal or bulk state is achieved in aggregates occupying a region whose size usually is of the order of a few molecular diameters, thus of the order of $3 \times 10^{-10} \mathrm{~m}$. On the curve itself, a bulk state is a mixture, with regions, gaseous or liquid, visible to the naked eye, whose size, in terms of molecular diameters, is therefore effectively infinite.

The size required in order for quantities like the density to be defined is usually, for statistical reasons, referred to as the correlation length and denoted by $\xi$. It depends on the pressure and the temperature, $\xi=\xi(P, T)$, and 
becomes infinite at the critical point $B$ because, for the reasons given, it is infinite along the curve. Thus the scale on which the thermal fluctuations occur grows as the critical point is approached, eventually reaching and surpassing the wavelength of visible light, about $5 \times 10^{-7} \mathrm{~m}$.

Although our initial discussion is for water, because it is so common, it may not be, as the following citation suggests, the best substance with which to conduct experiments around the critical point. For reasons described clearly and simply in [S], they are very difficult.

The optical phenomena, known as critical opalescence, that result from the increase in correlation length are quite colorful and very famous. Unfortunately, the best photographs and slides have never, to our knowledge, been published. We refer the reader to the cover of the June 10, 1968 issue of Chemical and Engineering News for the only color reproduction known to us. It would be useful, and would clear up many common misconceptions, if photographs illustrating the brownish-orange stage of Michael Fisher's description of critical opalescence for carbon dioxide in [F2] were published:
"if the carbon dioxide, which is quite transparent in the visible region of the spectrum, is illuminated from the side, one observes a strong intensity of scattered light. This has a bluish tinge when viewed normal to the direction of illumination, but has a brownish-orange streaky appearance, like a sunset on a smoggy day, when viewed from the forward direction (i.e., with the opalescent fluid illuminated from behind). Finally, when the temperature is raised a further few tenths of a degree, the opalescence disappears and the fluid becomes com- pletely clear again."

We review as briefly as we can, in a form suitable for mathematical consumption, the conceptual conclusions from the experiments. Our discussion, which begins with scaling and universality, is taken from [F1] and the companion survey $[\mathrm{H}]$ of experimental results. The notion of renormalization had not appeared in the theory at this stage. We stress at the outset that scaling is one conclusion from the experimental evidence, and universality a second. Renormalization is, for the moment, a largely heuristic mathematical argument to explain them both.

Although the details of the phase diagram varies from substance to substance, it remains qualitatively the same, and the behavior of the correlation length $\xi$ does not change. As far as can be determined it behaves near the critical point like a power of the distance $\rho$ from the critical point

$$
\xi \sim \rho^{-\nu} .
$$

On the curve defined by setting $T$ equal to $T_{c}$, the parameter $\rho$ is $\left|P-P_{c}\right|$; on that defined by setting $P=P_{c}$ it is $\left|T-T_{c}\right|$.

The equation (2.2b) is another instance of scaling that can immediately be compared with that of (2.1a). The correlation length is the size of the sample that is necessary for local statistical irregularities to be disregarded, so that the substance is in a normal or bulk state. For percolation, when the parameter $p$ is not equal to $p_{c}$ this is the size at which the conclusions of the first or the second part of the theorem take effect, thus for which $\pi_{h}^{n}(p)$ is very close either to 0 or to 1 . Since $A_{n}$ is the derivative at $p_{c}$ this requires, according to the 
third part of the theorem, that the absolute value of $A_{n}\left(p-p_{c}\right)$ be a number $B$ bounded away from 0 . The smallest $n$ at which this occurs is a candidate for the correlation length. The two relations $\xi=n$ and

$$
A_{n}\left|p-p_{c}\right|=B \text {, }
$$

together with $(2.2 b)$ yield

$$
\left|p-p_{c}\right|^{-1} \sim A_{n} \sim n^{\frac{1}{\nu}}=\xi^{\frac{1}{\nu}},
$$

or $\xi \sim\left|p-p_{c}\right|^{-\nu}$.

Although the critical exponent $\nu$ is the obvious one for percolation, and fundamental in general, it is one of the most difficult to measure experimentally. For the liquid-gas transition in pure fluids Fisher asserts ([F2]) that it has a value in the range 0.55 to 0.70 , implicitly suggesting that its value is independent of the fluid, thus universal. Since this is certainly not the value $\frac{4}{3}$ that appears to be correct for percolation, there must certainly be more than one universality class.

Although the phenomena of universality and scaling were discovered prior to the introduction of the renormalization group, it is easier to persuade the mathematical reader of the delicacy of the notion of universality classes into which real substances and models are supposed to fall, if it is explained immediately that they are expected to correspond to the stable manifolds of unstable fixed points of the renormalization group transformation that has not yet been described. Since these fixed points may not be isolated, and the transformation may draw a point on the stable manifold of a fixed point $Q$ very close to another fixed point $Q^{\prime}$ before drawing it to $Q$, the difficulties of classification and recognition of these classes are formidable even at the conceptual level ([F2]). Experimental uncertainties $([\mathrm{H}])$ only increase them.

Whether one is treating real systems or mathematical models, there are usually a number of critical indices, some of which will be introduced explicitly later, associated to a critical point of the system or model. It will also be explained, that within a universality class, they have equal values. The real systems are of various types: the fluids already discussed with the liquid-gas transition; magnetic systems, either ferromagnetic or antiferromagnetic; mixtures of two fluids; and many others. They all presumably admit an exact, although enormously complicated mathematical description. The best known mathematical model of a classical physical system is the Ising model of ferromagnetism. There are also models, like percolation, in which a classical thermodynamic interpretation of the parameters is somewhat factitious. The universality classes cut across the classification by these features. The principal factor is the dimension; and certain coarse features of the local interactions, such as isotropy or lack of it, the major secondary factor. Other possible secondary factors are noted in $\S 2.6$ of [F2].

Our principal concern is with percolation in dimension two; so the first factor is fixed. Moreover there is no interaction present in percolation; so the second factor is absent. The variations in lattice structure and in percolation type, whether on sites or bonds, that were described above appear not to affect the universality class of two-dimensional percolation.

For systems or models to which the classical thermodynamic paradigm is applicable, there are two quite different types of variables: those that in sta- 
tistical mechanics appear as parameters in the hamiltonian (strictly speaking, otherwise the temperature is not included, in the Boltzmann weight), and in thermodynamics are applied externally and naturally subject to the control of the experimenter, the temperature and pressure for a fluid, the temperature and the applied magnetic field for a magnet; and those that it is more natural to express as amounts per unit volume or lattice site. We refer to the first as external variables and to the second as internal. Typical internal variables are density, entropy and magnetization per unit volume, or in lattice models per site. They are given statistically as averages and thermodynamically as derivatives of a function $f$, the free energy per unit volume or site, with respect to a dual external variable.

There are also two types of critical indices, although they are not always clearly distinguished: those associated to thermodynamic quantities; and those that are defined at the molecular level and usually studied optically, or at least electromagnetically. Although analogues of those of the first type can also be defined for percolation, the analogues of those of the second type are the more natural in the context of this paper. The notion of scaling is more easily explained for the first; so we begin with them.

Since our treatment follows [F1] and [F2], it is more convenient to work with a ferromagnetic system. The pertinent external variables are the temperature $T$ and the applied magnetic field $H$. In the phase diagram Figure $2.2 \mathrm{~b}$ only the curve $C$ and the point $B$ remain. The curve $C$ is an interval, $H=0, T \leq$ $T_{c}$, and $B$, the Curie point, is $\left(T_{c}, 0\right)$. The liquid-gas transition is replaced by the possibility of spontaneous magnetization along $C$ whose sign, but not magnitude, depends on whether we approach $C$ from above or below. (Strictly speaking, the variable $H$ is a vector, and so is the magnetization, but this is a possibility best ignored.)

If we choose as independent variables near $B$ the difference $t=T-T_{c}$ as well as $h=H$, so that the critical point has coordinates $(0,0)$, then the free energy $f=f(t, h)$ satisfies (approximately) an equation

$$
f(t, h)=b^{-d} f\left(b^{\lambda_{1}} t, b^{\lambda_{2}} h\right) .
$$

This equation is experimental, and as explained by Fisher, was realized by $\mathbf{B}$. Widom to be a concise and illuminating manner of expressing scaling laws. The number $b$ is to be greater than 1 but otherwise arbitrary, and $\lambda_{1}, \lambda_{2}$ are two critical exponents in terms of which all others can be expressed. For reasons that will be discussed later, $1 / \lambda_{1}$ is identified with $\nu$. The quotient $\lambda_{2} / \lambda_{1}$ is denoted $\Delta$. We observe that the notation for critical indices is consistent from reference to reference, so that, when Fisher ([F1,F2]) and Grimmett ([G], especially $\S 7.1$ with which we urge the reader to compare the following discussion) use the same notation, they are referring to analogous exponents. The integer $d$ is the dimension. Thus for the moment it is 3 . Later, when we return to percolation, it will be 2 .

There are four critical indices $\alpha, \beta, \gamma, \delta$ associated to thermodynamic quantities. The induced magnetization per unit volume is given by

$$
M=\partial f / \partial h \text {. }
$$

It is in essence the ferromagnetic analogue of the density. Taking the derivative with respect to $h$ in $(2.2 \mathrm{c})$, and letting $h$ approach 0 , from above or below 
for the two limits may be different, we obtain

$$
M\left(t, 0_{ \pm}\right)=t^{\beta} M\left(1,0_{ \pm}\right), \quad \beta=d \nu-\Delta,
$$

upon setting $b^{-\lambda_{1}}=t$. Thus near the critical point, the spontaneous magnetization is (approximately!) a homogeneous function of $t=T-T_{c}$.

The magnetic susceptibility or the rate of variation of $M$ with $H$, an analogue of the compressibility of equation (2.2a), is $\partial M / \partial h$. Thus at $h=0$ it is homogeneous of degree $-\gamma=\beta-\Delta$ as a function of $t$. The third critical index $\delta$ describes the behavior of $M$ as a function of $h$ along the curve $T=T_{c}$ or $t=0$. Clearly

$$
M(0, h)=h^{\frac{1}{\delta}} M(0,1), \quad \delta=\Delta / \beta .
$$

Observe that the limit as $t \rightarrow 0$ is the same from both sides.

The specific heat is, apart from a factor, the second derivative of $f$ with respect to $t$. Thus at $h=0$ it behaves like $t^{-\alpha}$ with $\alpha=2-d \nu$.

There are two standard critical indices defined at the molecular level, and therefore statistically: the index $\nu$ and a second index $\eta$. Away from the critical point, correlation functions typically decrease exponentially in space, as (very roughly) $\exp (-|x-y| / \xi)$, where $x$ and $y$ are two points in space and $\xi$ is the correlation length. At the critical point $\xi$ becomes infinite and this rapid decay is replaced by a slower decay $|x-y|^{2-d-\eta}$. Thus $\eta$, in contrast to the other indices, refers specifically to behavior at the critical point itself, rather than in a neighborhood of it.

To express $\nu$ and $\eta$ in terms of $\lambda_{1}$ and $\lambda_{2}$ demands a more sophisticated discussion than that for the other four critical indices ([F1]). The result is that

$$
\nu=\frac{1}{\lambda_{1}}, \quad \eta=2-\frac{\gamma}{\nu}=2+d-2 \lambda_{2}
$$

As a consequence, $\lambda_{1}, \lambda_{2}$, and all the other critical indices can be expressed in terms of $\nu$ and $\eta$.

Scaling is a statement about a specific physical system or model. Universality, which asserts that the critical indices are constant (or nearly so) on broad classes is a second, quite distinct assertion. The evidence for both consists largely either of experimental data or the results of computations for specific models.

Theoretical justification is scant. The renormalization group yields, however, some insight into (2.2c). It is easiest to consider lattice models of ferromagnetism, in which each site of the lattice $L \subset \mathbb{R}^{d}$ of $\S 2.1$ is taken to be occupied by a magnet, whose magnetization and orientation may or may not be sharply constrained. In the widely studied Ising model it is constrained to take either of two opposing orientations and to be of fixed magnitude, thus effectively to assume only the values \pm 1 . Constraints are unimportant at the moment; it is rather the geometry that counts. Rather than taking only simple magnets at the sites, we could also allow some complicated system formed by a collection of mutually interacting magnets to be the object attached to the site. Then the interaction between the objects at neighboring sites, or more generally sites in close proximity, will be the resultant of the interaction between the magnets in the systems attached to the two sites. The advantage of the more general formulation is that such systems can be composed. 
This is the essence of renormalization, and the expository problem at this point is to provide the reader with some idea of this composition, because it informs all our investigations, but without prejudicing in any way the precise form it is to take. It is not the least of our purposes (as in [L1,L2]) to search for novel, perhaps even mathematically more tractable definitions of the composition.

We begin vaguely. The systems attached to the sites at the corners of a $d$ dimensional cube can be fused into a single system. Starting therefore with one model $M$, we can construct a second $M^{\prime}=\Theta(M)$ by attaching to the site $x=\left(x_{1}, x_{2}, \ldots, x_{d}\right) \in \mathbb{R}^{d}$ the system obtained by fusion from those at the sites

$$
x^{\prime}=\left(2 x_{1}+\epsilon_{1}, 2 x_{2}+\epsilon_{2}, \ldots, 2 x_{d}+\epsilon_{d}\right),
$$

the numbers $\epsilon_{i}$ each taking the values 0 and 1 .

Consider, as in $\S 2.1$ the system formed by the magnets on the sites inside a large block $S_{n}$ of side $n$. If $n=2^{m}$ the system is obtained by starting with independent systems of side 1 , putting $2^{d}$ together to form a block of side 2 , and then iterating the procedure $m$ times. Thus the model $M$ in the bulk can be considered to be the model $M^{(m)}=\Theta^{m}(M)$. Since the basic assumption of statistical mechanics is that the properties of sufficiently large finite systems are essentially those of infinite systems, we might suppose that $M^{(m)}$ and $M^{(m+1)}$ were essentially the same; thus, that $M^{(m)}$ was a fixed point of $\Theta$.

The mapping $\Theta$ is a renormalization, so that fixed points of the (semi-)group it generates appear to be objects of central importance. Universality can now be formulated as the assertion that there are few fixed points of $\boldsymbol{\theta}$ pertinent to the systems of interest.

The first, obvious difficulty is that to define $\Theta$ we have had to allow our system to grow more complex, so that a problem of closure presents itself. The second, less obvious, is that although what may be one of the major factors responsible for universality is implicit in the definition of $\boldsymbol{\Theta}$, nothing in the definition provides any insight into the mechanism by which it prevails over the details of the local interaction. Namely, the propagation in $M^{\prime}=\Theta(M)$ is across the walls separating the $2^{d}$ constituents of the composite system, and as we iterate $\Theta$ the number and nature of the paths along which the system at one site influences those at another depend strongly on the dimension $d$, and this multiplicity appears to dominate all other factors.

In one dimension the propagation is linear, and the problems can usually be formulated in terms of Markov processes, so that an analysis in terms of the renormalization group, although instructive, is from a strictly mathematical point of view not necessary. In two and more dimensions, it is one of the most effective methods for obtaining a handle on the qualitative behavior of the system at a critical point, but the problem of closure becomes more severe $([F 2, \S 5.6])$.

Although the crossing probabilities of the next section are the coordinates whose utility in the study of renormalization we are examining, standard treatments more often use, in one form or another, the external variables that appear in the hamiltonian. A simple example due to Nelson and Fisher and taken from $\S 5.2$ of [F2] admits a precise definition of renormalization, and may give the reader a clearer notion of the way it functions.

It is the Ising model in one dimension. Consider a finite collection of integers 
$S_{N}=\{i \mid-N \leq i \leq N\}$. The possible states of the model are the functions $s$ on $S_{N}$ with values in $\{ \pm 1\}$. The energy of a state is given by the hamiltonian function,

$$
H_{0}(s)=K_{0} \sum_{-N \leq i \leq N-1} s_{i} s_{i+1}+h_{0} \sum_{-N \leq i \leq N} s_{i}+C_{0} \sum_{-N \leq i \leq N} 1 .
$$

In statistical mechanics the free energy per site is given as the quotient

$$
-k T \ln \left(\sum_{s} \exp \left(-\frac{1}{k T} H_{0}(s)\right)\right) /(2 N+1)
$$

the sum running over all states $s$. (The factor $k$ that ensures that the argument of the exponential function is dimensionless is called the Boltzmann constant.) Emphasis is therefore often put on the partition function

$$
Z_{N}(H)=\sum_{s} \exp \left(-\frac{1}{k T} H_{0}(s)\right)=\sum_{s} \exp (-H(s)),
$$

where we have set

$$
H(s)=H(s ; K, h, C)=K \sum_{-N \leq i \leq N-1} s_{i} s_{i+1}+h \sum_{-N \leq i \leq N} s_{i}+C \sum_{-N \leq i \leq N} 1,
$$

with

$$
K=\frac{K_{0}}{k T}, \quad h=\frac{h_{0}}{k T}, \quad C=\frac{C_{0}}{k T} .
$$

It is appropriate to refer to $K, h$, and $C$ as the external variables. (There is, as observed, a slight abuse of terminology here. The parameter $T$ appears in $K$ but not, strictly speaking, in the original hamiltonian.) Observe that in statistical mechanics the probability of the state $s$ is taken to be equal to

$$
\exp (-H(s)) / Z_{N}(H) \text {. }
$$

We could fuse the systems at $s_{2 i}$ and $s_{2 i+1}$ so that the system attached to the site $i$ then consisted of two simple magnets interacting through the energy $K^{\prime} s_{2 i} s_{2 i+1}$, but this changes the nature of the system, so that problems of closure arise. Rather the emphasis is put on calculating the partition function as a function of the three external parameters. Fix the values of the $s_{2 i}$ so that the local state is determined at the even sites, and take the sum in $(2.2 \mathrm{~d})$ over the two possible values of $s_{2 i+1}$ at all the odd sites. If we define $s^{\prime}$ by $s_{i}^{\prime}=s_{2 i}$, the result may be written as

$$
\sum_{s^{\prime}} \exp \left(-H^{\prime}\left(s^{\prime}\right)\right)=Z_{N^{\prime}}^{\prime}, \quad N^{\prime}=N / 2,
$$

if a certain fuzziness at the endpoints is accepted. It can be expected to resolve itself in the limit of large $N$. The problem of closure arises because the hamiltonian $H^{\prime}$ may be of quite a different form than $H$, so that the calculation transfers us to a larger space of hamiltonians, and no real simplification has been achieved.

The advantage of the example (we stress that it is very unusual), achieved only at the cost of abandoning the initial fusion and summing in an arbitrary manner over the states at the odd-numbered sites, is that $H^{\prime}$ turns out to be of the form

$$
H^{\prime}\left(s^{\prime}\right)=H\left(s^{\prime} ; K^{\prime}, h^{\prime}, C^{\prime}\right)
$$


if

$$
\begin{aligned}
w^{\prime} & =w^{2} x y^{2} /(1+y)^{2}(x+y)(1+x y) \\
x^{\prime} & =x(1+y)^{2} /(x+y)(1+x y) \\
y^{\prime} & =y(x+y) /(1+x y) .
\end{aligned}
$$

The three parameters appearing here are given by

$$
w=e^{4 C}, \quad x=e^{4 K} \text { and } y=e^{2 h} .
$$

Thus $\Theta$ appears here simply as the transformation

$$
(K, h, C) \rightarrow\left(K^{\prime}, h^{\prime}, C^{\prime}\right) .
$$

In order to examine the physical properties of the hamiltonians $H$, one can use the correlation length $\xi(H)$. Let $f(i)$ be the (limit for large $N$ of the) probability that $s_{0}$ and $s_{i}$ have the same orientation and let $\xi(H)$ be a measure of the width of this distribution, say the largest value $|i|$ such that $f(i)>f_{0}$ for some constant $f_{0}$. If we limit ourselves to the even integers $i$, the value of $\xi$ should not change seriously, so that partial summation over the odd sites does not affect the correlation length. On passing from $H$ to $H^{\prime}$ we relabeled, denoting $s_{2 i}$ be $s_{i}^{\prime}$. The result is therefore that

$$
\xi\left(H^{\prime}\right)=\frac{1}{2} \xi(H) \text {. }
$$

The renormalization-group transformation $\boldsymbol{\theta}$ is, in this example, the process of "decimation", thus of removing one-half the sites, followed by a shrinking of the lattice scale, and the replacement of $H$ by $H^{\prime}$. It decreases the correlation length by the factor $1 / 2$. The space of models is parametrized by a subset of $\mathbb{R}^{3}$.

We have claimed that the fixed points of the map $\boldsymbol{\theta}$ are of major interest. At a fixed point $(w, x, y)$ of the map $\Theta$ the hamiltonian $H=H(w, x, y)$ would be invariant under $\Theta$ and its correlation length would have to satisfy

$$
\xi(H)=\frac{1}{2} \xi(H),
$$

so that $\xi(H)=0$ or $\xi(H)=\infty$. For the physical reasons explained at the beginning of this section, it is the solutions of the second type that yield critical points. They are examined in more detail in $§ 5.3 .2$ of [F2] and in [NF].

The simplicity of this one-dimensional example is misleading. For the twodimensional Ising model, decimation appears to require the introduction of a further variable (in addition to $K, h$ and $C$ ) describing the interaction of second nearest neighbors. Iterating the decimation will require more and more variables, so that the problem of closure manifests itself clearly. This behavior, and not that of the example, is typical. What one expects in general is that (2.2e) will be replaced by an equation

$$
H^{\prime}\left(s^{\prime}\right)=H\left(s^{\prime}, K^{\prime}, h^{\prime}, C^{\prime}\right)+H^{\prime \prime}\left(s^{\prime}\right),
$$

in which $H^{\prime \prime}\left(s^{\prime}\right)$ is small, and at each step smaller, eventually becoming irrelevant.

In [L1] and [L2] the emphasis is on approximations to the "true" $\boldsymbol{\theta}$ by a collection of increasingly complex transformations that act on finite-dimensional spaces and whose first members permit close study. Since these approximating 
transformations are the reason for our emphasis on crossing probabilities, we shall briefly describe them in the next section.

We first return briefly to the equation (2.2c) imagining ourselves at a fixed point. It will be associated to a complicated system, so that there will be many more external variables than merely $h$ and $T$ (or $t$ ) needed to determine the local interactions and therefore the free energy per site but, typically, they will be irrelevant. Mathematically this means that they are variables along the directions in which $\Theta$ is contracting. (In the example, $K$ is just another form of $T$, but as often happens, there is more than one supplementary relevant variable, not only $h$ but in addition $C$. The irrelevant variables, had they appeared, would be those defining $H^{\prime \prime}$.) If we ignore these irrelevant directions then $\Theta$ will be roughly of the form

$$
(t, h) \rightarrow\left(2^{\lambda_{1}} t, 2^{\lambda_{2}} h\right) \text {. }
$$

Since renormalization obviously multiplies the free energy per site by $2^{d}$, we obtain, upon ignoring the other, irrelevant variables, the equation

$$
2^{d} f(t, h)=f\left(2^{\lambda_{1}} t, 2^{\lambda_{2}} h\right) \text {. }
$$

Iterating we obtain (2.2c) with $b$ equal to a power of 2 . In other words, scaling can be recovered from renormalization group arguments. So can universality, because the two indices $\lambda_{1}$ and $\lambda_{2}$ are associated to the fixed point, not to the model with which the iteration begins.

It is implicit in these equations that for

$$
|t|+|h|=1
$$

the value of $f$ is neither very large nor very small. Both $\lambda_{1}$ and $\lambda_{2}$ are positive. Since $f$ is the free energy per site, it is clear from $(2.2 \mathrm{c})$ that for $t$ and $h$ very small, the side, $b$, of the block needed in order that the total free energy be of order 1 is given by the condition that $b^{d} f(t, h) \sim 1$, thus that $b=t^{-\lambda_{1}}$ or $b=h^{-\lambda_{2}}$. If $h \ll t$, the first condition gives the smaller $b$ and the relation $\nu=1 / \lambda_{1}$. For more serious demonstrations of this relation the reader is referred to $[\mathrm{F} 1]$.

We observe in passing that $\lambda_{2}$ can be larger than $\lambda_{1}$ so that we see no very strong reason that

$$
f(t, h)=b^{-d} f\left(b^{\lambda_{1}}(t+c h), b^{\lambda_{2}} h\right),
$$

with a constant $c$ might not be preferable to $(2.2 \mathrm{c})$. We have followed convention.

2.3. Crossing probabilities. Percolation is not a model of a classical physical system with a thermodynamic interpretation, and the finite models that appear later in this section are stripped of many features of such models; so their value is uncertain. Their purpose, as we have already remarked, is to provide a model of the dynamics of renormalization that is accessible mathematically, and that reveals the essence of the processes involved. It is still far from certain that this purpose will be achieved, but to defend it as a goal we cite a phrase from Fisher's description in $[F 1, \S 1.2]$ of the role of models:

“... the aim of the theory of a complex phenomenon should be to elucidate which general features ... of the system lead to the most characteristic and typical observed properties.” 
We have deleted the words "of the Hamiltonian" because we focus on percolation, deliberately to avoid all problems caused by the hamiltonian. Those caused by the multiple paths along which effects are propagated in two dimensions remain, so that Fisher's demand that initially:

“... one should aim at a broad qualitative understanding, successively refining one's quantitative grasp of the problem" is met.

The rest of the paper concentrates on two-dimensional percolation. The two hypotheses presented in $\S 2.4$ relate the critical behavior of a large class of models. Before stating these hypotheses we shall first introduce the models they are likely to describe and then extend the notion of the horizontal crossing probability $\pi_{h}$ to larger families of geometrical data.

Let $\mathscr{G}$ be a graph embedded in $\mathbb{R}^{2}$. As in the introduction, we refer to its vertices as sites and to its edges as bonds. It is a periodic graph $[\mathrm{K}]$ if it satisfies the following conditions:

(1) $\mathscr{G}$ contains no loops (in the graph-theoretical sense);

(2) $\mathscr{G}$ is periodic with respect to translations by the elements of a lattice $L$ in $\mathbb{R}^{2}$ of rank two;

(3) the number of bonds attached to a site in $\mathscr{G}$ is bounded;

(4) all bonds of $\mathscr{G}$ have finite length and every compact set of $\mathbb{R}^{2}$ intersects finitely many bonds of $\mathscr{G}$;

(5) $\mathscr{G}$ is connected.

Let $\mathscr{S}$ be the set of sites of $\mathscr{G}$ and $p: \mathscr{S} \rightarrow[0,1]$ a periodic function, thus a function invariant under the translations from $L$. As before we allow each site $s \in \mathscr{S}$ to be in either state 0 (closed) or 1 (open) and we define a measure $P_{s}$ on the set $\{0,1\}$ by the equations $P_{s}(0)=1-p(s)$ and $P_{s}(1)=p(s)$. Finally we introduce the set of configurations $X$ on the graph $\mathscr{G}$ as the product $\prod_{\mathscr{S}}\{0,1\}$ and endow $X$ with the product measure $m$ of the various $P_{s}$. A model $M=M(\mathscr{G}, p)$ is defined as the set of data $\{\mathscr{G}, p, X, m\}$. We shall refer to these models as the class of graph-based models. Observe that for a given $\mathscr{G}$ the family of possible functions $p$ form a compact set in some finitedimensional space.

The model $M_{0}$ corresponds to a graph constructed of the vertices $\mathbb{Z}^{2}$ with edges between nearest neighbors and the function $p$ constant on all sites. The definition also includes the models of percolation by sites on triangular and hexagonal lattices. To include models of percolation by bonds one associates ([K]) to a graph $\mathscr{G}$ its matching graph $\tilde{\mathscr{G}}$. The sites of $\tilde{\mathscr{G}}$ are the midpoints of the bonds of $\mathscr{G}$; two distinct sites $\tilde{s}_{1}$ and $\tilde{s}_{2}$ of $\tilde{\mathscr{G}}$ are joined if and only if the corresponding bonds $b_{1}$ and $b_{2}$ of $\mathscr{G}$ are attached to a common site. A periodic function $p$ on the bonds of $\mathscr{G}$ leads naturally to a periodic function $\tilde{p}$ on the sites of $\tilde{\mathscr{G}}$ and we can therefore replace percolation by bonds on $\mathscr{G}$ by percolation by sites on $\tilde{\mathscr{G}}$. Percolation by bonds on a square lattice where horizontal bonds are open with probability $p_{h}$ and vertical ones with a different probability $p_{v}$ is an example of a model for which the probability function is not constant.

The hypothesis of universality in $\S 2.4$ has only been examined numerically for a few models. If we were eager to be precise, we might suggest the class of graphbased models as the appropriate class for which to formulate the hypothesis. 
Such precision is inappropriate at this stage. In particular, other models will very likely fall into the same universality class.

That this is so for a model based on an aperiodic graph whose sites and bonds are defined by a Penrose tiling on the plane is indicated by the results of [Y]. Thus the condition of periodicity is excessively prudent. Models may also be defined without any reference to graphs, for example by randomly placing unit disks on the plane $\mathbb{R}^{2}$ with a density $\delta$. If a rectangle is drawn on the plane, a horizontal crossing is a path from left to right on overlapping disks. The density $\delta$ plays the role of the probability $p$ that a site is open. (See $[G]$ for a discussion of the "snails on a lily pond" model.) The disks can be replaced by ellipses with uniform random orientation or, in the limit, by segments of length one. Results of $\mathrm{H}$. Maennel for crossing probabilities in this limiting case confirm that they are the same as those of $M_{0}$.

For graph-based models the notion of a cluster for a given state is simple. It is a maximal connected subset of the set of open sites. The universality emphasized in [U] is that of the crossing probabilities, the probabilities of events defined by a simple closed curve $C$ in the plane and by arcs $\alpha_{1}, \ldots, \alpha_{m}$, and $\beta_{1}, \ldots, \beta_{m}$, as well as $\gamma_{1}, \ldots, \gamma_{n}$ and $\delta_{1}, \ldots, \delta_{n}$ of $C$.

Let $A$ be a large constant and define $C^{\prime}$ and the intervals $\alpha_{i}^{\prime}, \beta_{i}^{\prime}, \gamma_{j}^{\prime}$, and $\delta_{j}^{\prime}$ to be the dilations, with respect to some fixed but irrelevant point in the plane, of $C$ and $\alpha_{i}, \beta_{i}, \gamma_{j}$ and $\delta_{j}$ by the factor $A$. In principle a given state admits a crossing inside $C^{\prime}$ from $\alpha_{i}^{\prime}$ to $\beta_{i}^{\prime}$ if there is cluster for this state whose intersection with the interior of $C^{\prime}$ intersects both $\alpha_{i}^{\prime}$ and $\beta_{i}^{\prime}$. Since $C^{\prime}$ is a curve, it might not contain any sites and it is in fact necessary to replace $C^{\prime}$, supposed to be not too irregular, by a band, and to thicken the intervals accordingly. Then there will be a crossing between $\alpha_{i}^{\prime}$ to $\beta_{i}^{\prime}$ if there is an open path inside $C^{\prime}$ from the thickening of these two intervals. For large $A$ the choice of band, provided it is relatively narrow, is irrelevant. We describe specific conventions when discussing the experiments.

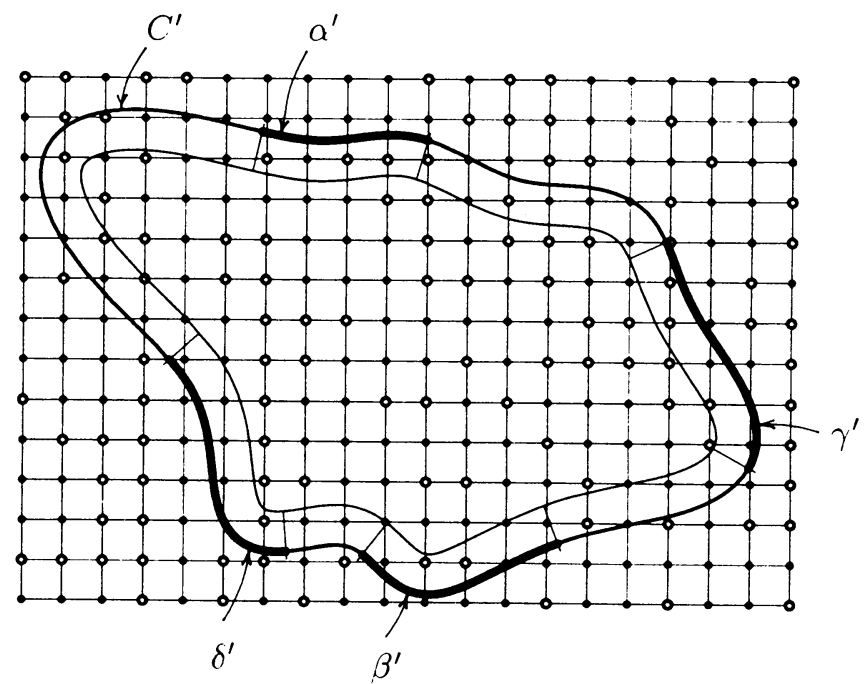

Figure 2.3a. Data $(C, \alpha, \beta, \gamma, \delta)$ defining the event $E$. 
With appropriately chosen conventions we can therefore define

$$
\pi_{A}\left(C, \alpha_{1}, \ldots, \alpha_{m}, \beta_{1}, \ldots, \beta_{m}, \gamma_{1}, \ldots, \gamma_{n}, \delta_{1}, \ldots, \delta_{n}\right)
$$

the probability that there are crossings in the interior of $C^{\prime}$ from $\alpha_{i}^{\prime}$ to $\beta_{i}^{\prime}$ for $1 \leq i \leq m$ but no crossing from $\gamma_{j}^{\prime}$ to $\delta_{j}^{\prime}$ for $1 \leq j \leq n$. One may suppose that these conventions will be such that they do not affect the existence or the value of the limit

$$
\lim _{A \rightarrow \infty} \pi_{A}\left(C, \alpha_{1}, \ldots, \alpha_{m}, \beta_{1}, \ldots, \beta_{m}, \gamma_{1}, \ldots, \gamma_{n}, \delta_{1}, \ldots, \delta_{n}\right)=\pi(E, M) .
$$

We take $E$ as an appropriate abbreviation for the event (or rather events since we took a limit over dilations) defined by $C, \alpha_{i}, \beta_{i}, \gamma_{j}$ and $\delta_{j}$. The horizontal crossing probability $\pi_{h}$ defined for $M_{0}$ in the introduction is a special case of $\pi\left(E, M_{0}\right)$. The curve $C$ is a square and only two arcs $\alpha$ and $\beta$ are chosen, the left and right sides.

A natural extension ([K], $[\mathrm{AB}])$ of the theorem of $\S 2.1$ is that, a family of models $M(\mathscr{G}, p)$, parametrized by the function $p$, is constituted by two open sets, one for which the limit (2.3a) is always 1 and one for which it is always 0 ; a third subset, the set of critical probabilities, separates the other two and is such that the limit (2.3a) (if it exists) lies in general between 0 and 1 . Presumably the limit does exist even for the critical probabilities, but this has not yet been established. The two simplest models, percolation by sites and bonds on a square lattice, for which $p$ varies over an interval, are critical for a single appropriate choice $p_{c}$ of $p$. Hence the two open sets are $\left[0, p_{c}\right)$ and $\left(p_{c}, 1\right]$ and the critical subset is $\left\{p_{c}\right\}$. For percolation by sites the value of $p_{c}$ is known empirically to be $0.5927460 \pm 0.0000005$ [Z]; for percolation by bonds it is known theoretically [K] to be $\frac{1}{2}$.

All our numerical work, as well as the hypotheses underlying it, is predicated on the existence of these limits, that we now take for granted. Moreover our models are from now on supposed to be critical.

Since our investigations were initially prompted by the desire to provide empirical foundations for the definitions of the finite models of [L1] and [L2], we review those definitions briefly. We shall also need to have them at our disposal in $\S 2.5$.

Let $S$ be a square whose sides have been divided in $l$ equal intervals. There are $4 l(4 l+1) / 2$ pairs of intervals. Let $\mathscr{P}$ be the set of these pairs. A configuration $x$ for this model is obtained by specifying which pairs are connected and which ones are not. Assign them respectively the values +1 and -1 . The space $A$ of configurations is then a set of functions from $\mathscr{P}$ to $\{+1,-1\}$. (There are technical constraints on the configurations that need not be described here.) Therefore each element of $A$ is an event $E$ whose defining curve $C$ is a square. (According to the hypothesis of conformal invariance, all crossing probabilities can be obtained from those for this case. See $\S 2.4$ )

There is a natural transformation $\Theta_{A}: A \times A \times A \times A \rightarrow A$ that is similar to the renormalization-group transformation $\boldsymbol{\theta}$ of $\S 2.2$. To construct $\boldsymbol{\Theta}_{A}$ one first juxtaposes four elements of $A$ so that they form a larger square with $2 l$ 
subdivisions on its sides. These intervals are then fused in pairs so that each side of the larger square contains $l$ intervals. Finally these new intervals are connected by composing the "paths". Suppose, for example, that $\alpha$ and $\beta$ are connected intervals in one of the original squares and $\mu$ and $\nu$ are also connected in another one. If $\beta$ and $\mu$ turn out to be in the interior of the larger square formed upon juxtaposition and are coincident, then the larger intervals containing $\alpha$ and $\nu$ in this square will be connected. See Figure 2.3b for an example.
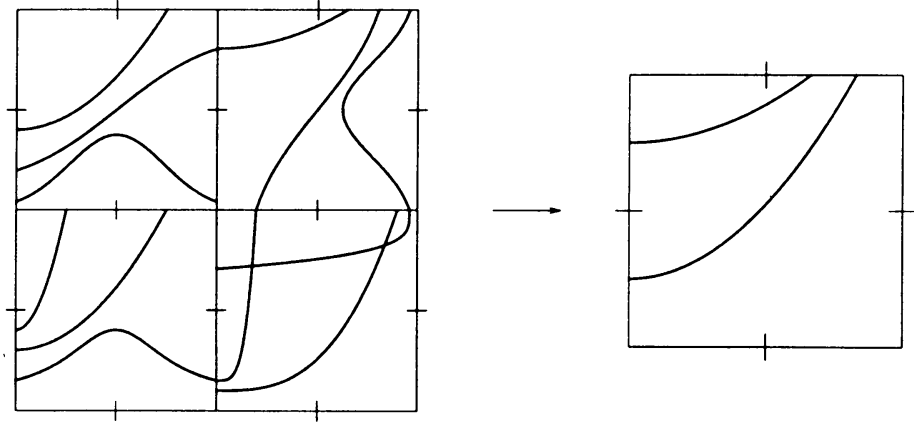

FIGURE 2.3b. An example of the transformation $\boldsymbol{\Theta}_{A}$ : $A \times A \times A \times A \rightarrow A$ for a finite model.

If $\mathfrak{X}$ is the set of measures on $A, \Theta_{A}$ can be used to define a map $\Theta_{\mathfrak{X}}: \mathfrak{X} \rightarrow$ $\mathfrak{X}$. Since $\mathfrak{X}$ is a simplex in a finite-dimensional space, the question of finding fixed points of $\boldsymbol{\Theta}_{\mathfrak{X}}$ and studying their nature is well-posed.

2.4. The two hypotheses. Although Aizenman prefers to distinguish between the hypothesis of universality and that of conformal invariance, regarding the first as commonly accepted, even in the form in which we state it, we prefer for the sake of clarity as well as for the reasons already rehearsed in [U] to state them in a less invidious form. The purpose of [U] was to show that the probabilities $\pi(E, M)$ were independent of $M$, provided the model satisfied some simple conditions of symmetry. This is a form of universality. To state the general form we observe that the group $G L(2, \mathbb{R})$ acts independently on the models and on the events. (From now on we restrict ourselves to the class of graph-based models.)

A model with sites $\{s\}$ and bonds $\{b\}$ is sent by $g \in G L(2, \mathbb{R})$ to the model with sites $\{g s\}$ and bonds $\{g b\}$, the probability function $p$ being transferred directly from the old sites and bonds to the new. The lattice $L$ defining the periodicity is then replaced by $g L$. The group $G L(2, \mathbb{R})$ acts on the events $E$ as well. We shall write $g E$ for the event obtained from the data $\left(C, \alpha_{i}, \beta_{i}, \gamma_{j}, \delta_{j}\right)$ defining $E$ by letting $g$ act on each element of the data: $g E=\left(g C, g \alpha_{i}, g \beta_{i}, g \gamma_{j}, g \delta_{j}\right)$. By the definitions,

$$
\pi(g E, g M)=\pi(E, M)
$$


since transforming simultaneously the embedding of the graph $\mathscr{G}$ and the curve $C$ by the same linear transformation does not alter $\pi(E, M)$. On the other hand, the probabilities $\pi(E, g M)$ and $\pi(g E, M)$ are generally quite different from $\pi(E, M)$.

Hypothesis of Universality. If $M$ and $M^{\prime}$ are any two (graph-based) models of percolation there is an element $g$ in $G L(2, \mathbb{R})$ such that

$$
\pi\left(E, M^{\prime}\right)=\pi(E, g M)
$$

for all events $E$.

Those experienced readers who feel that this hypothesis is generally accepted, and not worth examining numerically, might ask themselves how much they are willing to stake on its validity in three dimensions - life, family, career? Less experienced readers will be more likely to notice just how strong the statement is, and therefore to be more skeptical. We ourselves have found an explicit enunciation a great aid to clear thinking.

In paragraph 3.4, we shall give an example of a model $M$. for which the matrix $g$ of the hypothesis has no elements equal to 0 .

The hypothesis obtains its full force only in conjunction with that of conformal invariance. Suppose that $J$ is a linear transformation of the plane $\mathbb{R}^{2}$ with $J^{2}=-I$. Then $J$ defines a complex structure on the plane, multiplication by $i$ being given by $x \rightarrow J x$. Once $J$ is fixed, the notion of a $J$-holomorphic map on an open subset of the plane can be introduced as well as that of an antiholomorphic map. If $g \in G L(2, \mathbb{R})$ and $J^{\prime}=g J g^{-1}$, then the map $\phi \rightarrow g \cdot \phi \cdot g^{-1}$ transforms $J$-holomorphic maps into $J^{\prime}$-holomorphic maps and $J$-antiholomorphic maps into $J^{\prime}$-antiholomorphic maps.

If $\phi$ is a transformation $J$-holomorphic in the interior of $C$ and continuous and bijective up to its boundary, which is just $C$ itself, then the event $\phi E$ is well defined; the transformation $\phi$ is simply applied to the data $\left(C, \alpha_{i}, \beta_{i}, \delta_{j}, \gamma_{j}\right)$ defining $E$. We may also apply a transformation $\phi$ that is antiholomorphic in the interior to $E$. The following hypothesis was in essence suggested by Michael Aizenman.

Hypothesis of conformal invariance. For every model $M$ there is a linear transformation $J=J(M)$ defining a complex structure such that

$$
\pi(\phi E, M)=\pi(E, M)
$$

for all events $E$ whenever $\phi$ is J-holomorphic or J-antiholomorphic in the interior of $C$ and continuous (and, for the moment, bijective) up to its boundary.

To understand the nature of the hypothesis, consider the model $M_{0}$ of percolation by sites on a square lattice. The complex structure for $M_{0}$ is, if the hypothesis is correct, the usual one defined by

$$
J_{0}=\left(\begin{array}{cc}
0 & -1 \\
1 & 0
\end{array}\right)
$$

and the associated holomorphic functions are the usual ones. 
Given an event $E$ we may choose $\phi$ so that $E^{\prime}=\phi E$ is defined by the the unit circle $C^{\prime}$ with centre at the origin and arcs on it. If for example $E$ is defined by the horizontal crossing of a rectangle, then the data on $C^{\prime}$ will be four points $a, b, c$, and $d$, the images of the four corners of the square under $\phi$, and $\alpha^{\prime}=\phi(\alpha)$ will be the circular arc between $a$ and $b$, and $\beta^{\prime}$ the circular arc between $c$ and $d$.

For numerical work it is easier to use the inverse of $\phi$, a Schwarz-Christoffel transformation

$$
\psi: w \rightarrow \int_{0}^{w} \frac{d u}{\sqrt{\left(u^{2}-v^{2}\right)\left(u^{2}-1\right)}}
$$

in which $v$ is a constant of absolute value 1 that depends on the aspect ratio of the rectangle. For a square, one can clearly take $v=\sqrt{-1}$. In the arguments of $\S 2.6$ the disk is replaced by the upper half-plane, and in $\S 3.5$ the hypothesis is implicitly reformulated for all unbounded regions.

If $M$ and $M^{\prime}$ are related by the first hypothesis then $J\left(M^{\prime}\right)=g J(M) g^{-1}$. Denote the identity transformation by $I$. The set of linear transformations

$$
H(J)=\left\{a I+b J \in G L(2, \mathbb{R}) \mid a^{2}+b^{2} \neq 0\right\}
$$

is the centralizer of $J$ in $G L(2, \mathbb{R})$, and is of index two in

$$
H^{\prime}(J)=\left\{h \in G L(2, \mathbb{R}) \mid h J h^{-1}= \pm J\right\}
$$

The group $H$ determines $H^{\prime}$ but only determines $J$ itself up to sign. If $J=$ $J(M)$ we write $H(J)=H(M)$ and $H^{\prime}(J)=H^{\prime}(M)$. It is clear that the element $g$ that appears in the hypothesis of universality is not uniquely determined, at best the class $g H^{\prime}(M)$ is determined. As we shall observe explicitly later, there is in fact no further ambiguity, so that the two hypotheses together imply that the image under

$$
\psi: M \rightarrow \prod_{E} \pi(E, M)
$$

of the set of all models in the product, over all events $E$, of the interval $[0,1]$ (a very, very large set) is a small subset that may be identified with the upper half-plane. Each model of the class defined in $\$ 2.3$ corresponds to point in the upper-half plane. All the crossing probabilities $\pi(E)$ of models corresponding to the same point are identical. Thus universality and the orthogonal invariance of $M_{0}$ reduce an apparently infinite-dimensional continuum of possibilities for the image of $\psi$ to a two-dimensional continuum. Without orthogonal invariance, this continuum would already be three-dimensional; so universality is the determining factor.

Those who have read $\S 2.2$ will notice that the universality of that section is quite different from that of this paragraph. Universality in $\S 2.2$ is that of 
critical exponents and they could all be expressed in terms of $\lambda_{1}$ and $\lambda_{2}$ that can themselves be interpreted as the logarithms of the dominant eigenvalues of the Jacobian matrix of a suitable renormalization transformation at a fixed point. This fixed point is not usually regarded as existing in a physical sense, and is therefore treated as a somewhat spectral object. The assumption implicit in the finite models mentioned in $\S 2.3$ is that the fixed point itself, at least for percolation, is a real physical and mathematical object whose coordinates are the crossing probabilities, so that not only the critical indices but also these probabilities are universal. They and not the critical indices are the objects of principal interest in this paper. Nevertheless, although - mathematically - the point and its coordinates have to be studied before the eigenvalues of a transformation fixing it, it is the critical indices whose universality is to be explained and that have attracted the most attention from physicists so far. It is by no means certain that for other problems than percolation there will be useful analogues of the crossing probabilities of $\S 2.3$, and even less clear that they will be physically significant.

Although we do not want the renormalization group to intrude too obstreperously on the discussion, we repeat, in order that there be no misunderstanding, that the crossing probabilities are not to be interpreted as coordinates of the model at a critical value of the parameters but as those of the fixed point to which it is attracted. This is what permits the image of the map $\psi$ to be of such a small dimension.

To be concrete the image $(2.4 \mathrm{c})$ is obtained as the collection

$$
\psi: M \rightarrow \prod \pi\left(E, g^{-1} M_{0}\right), \quad g \in G L(2, \mathbb{R}),
$$

where $M_{0}$ is a given model, and the half-plane is identified with

$$
H\left(M_{0}\right) \backslash G L(2, \mathbb{R}) .
$$

Observe that the action of $G L(2, \mathbb{R})$ on this homogeneous space is to the right and is given on coordinates by

$$
\pi(E, M) \rightarrow \pi(g E, M) .
$$

The image (2.4c) can be identified with the set of all possible groups $H(M)$, thus with the set of all translation-invariant conformal structures on the plane up to orientation.

In a certain sense the hypothesis of universality is subsumed under that of conformal invariance, because the relation (2.4a) may be written

$$
\pi\left(E, M^{\prime}\right)=\pi\left(g^{-1} E, M\right),
$$

and $g^{-1}$ is a translation-invariant conformal map from the structure defined by $H\left(M^{\prime}\right)$ to that defined by $H(M)$, thus, in general, between two different conformal structures. The two hypotheses are thus fused into one if the equation

$$
\pi\left(E, M^{\prime}\right)=\pi(\phi E, M)
$$

is supposed valid for any map $\phi$ that is defined on the interior of the curve $C$ determining $E$ and continuous up to its boundary, and takes the conformal structure attached to $M^{\prime}$ to that attached to $M$.

Since $H^{\prime}\left(M_{0}\right)$ contains the reflections in both axes as well as the permutation of the two axes, it must be the orthogonal group, and we can identify the image 
(2.4c) with the upper half-plane in such a way that $M_{0}$ corresponds to the point $i$. The action of $G L(2, \mathbb{R})$ is then

$$
\left(\begin{array}{ll}
a & b \\
c & d
\end{array}\right): z \rightarrow \frac{a z+c}{b z+d}
$$

when $a d-b c$ is positive, and is

$$
\left(\begin{array}{ll}
a & b \\
c & d
\end{array}\right): z \rightarrow \frac{a \bar{z}+c}{b \bar{z}+d}
$$

otherwise. Let $R$ be the group of four matrices

$$
\left(\begin{array}{cc} 
\pm 1 & 0 \\
0 & \pm 1
\end{array}\right)
$$

and $S$ the group generated by $R$ and the matrix

$$
\left(\begin{array}{ll}
0 & 1 \\
1 & 0
\end{array}\right) \text {. }
$$

A simple calculation shows that the points invariant under $R$ are the points on the imaginary axis, and that the only point invariant under $S$ is the point $i$ itself.

In [U] we studied only models that obviously yielded points invariant under $R$, and thus were implicitly confining ourselves to a one-dimensional curve, the imaginary axis, in an otherwise two-dimensional family.

2.5. More critical indices for percolation. As we saw in $\S 2.2$ it is natural in models and systems with a thermodynamic significance to emphasize the way in which the internal variables depend on the external ones, and thus to introduce the critical indices $\alpha, \beta, \gamma$ and $\delta$. Once we pass to other coordinates, or other models in which there is no natural choice of coordinates, it is no longer clear which are the principal critical indices.

The abstract possibility of blowing up or contracting the ill-defined space in which $\boldsymbol{\theta}$ operates creates even more ambiguity. Suppose, for example, that in some rough sense $\boldsymbol{\theta}$ operates in the neighborhood of a fixed point as

$$
\boldsymbol{\Theta}: \quad\left(t_{1}, t_{2}, t_{3}, \ldots\right) \rightarrow\left(2^{\lambda_{1}} t_{1}, 2^{\lambda_{2}} t_{2}, 2^{\lambda_{3}} t_{3}, \ldots\right),
$$

and that only $\lambda_{1}$ and $\lambda_{2}$ are positive, so that only the first two coordinates are relevant. If we allow ourselves that freedom, then blowing up, as usual in algebraic geometry, so that $\left(t_{1}, t_{2} / t_{1}, t_{3}, \ldots\right)$ or $\left(t_{1} / t_{2}, t_{2}, t_{3}, \ldots\right)$ become the coordinates, we replace $\lambda_{2}$ by $\lambda_{2}-\lambda_{1}$ or $\lambda_{1}$ by $\lambda_{1}-\lambda_{2}$, creating two fixed points from one, and perhaps changing the number of unstable variables.

For percolation itself, our preferred coordinates are the numbers $\pi(E, M)$ defined by crossing probabilities. These permit readily, as we saw in $\S 2.1$, the introduction of the critical index $\nu$. Although the critical indices $\alpha, \beta, \gamma$, and $\delta$ can be defined directly within percolation ([G]), that they are indeed the analogues of those of $\S 2.2$ is best seen as in [E2,§2] by treating percolation as the limit of an Ising model in a weak field. They do not have an obvious interpretation in terms of the crossing probabilities that are in this paper the primary objects.

This can perhaps be forgiven if we can at least interpret $\eta$, which we recall refers to behavior at criticality, in terms of crossing probabilities. To this end 
we borrow some standard conjectures from [G, Chap. 7], and use freely the notions of conformal invariance developed in Part Three. We work with the model $M_{0}$ at $p=p_{c}$.

Let $P(r)$ be the probability at $p=p_{c}$ that the origin is open and the cluster containing it also contains a point at a distance at least $r$ from the origin. It is believed $[G,(7.10,7.11)]$ that

$$
P(r) \sim r^{-1 / \rho}, \quad \rho=48 / 5 .
$$

If $z$ is a point in the lattice $\mathbb{Z}^{2}$ let $\tau(0, z)$ be the probability that the origin 0 is occupied and the cluster containing it also contains $z$. It is further suggested that

$$
\tau(0, z) \sim|z|^{-\eta}, \quad \eta=5 / 24 .
$$

This is the $\eta$ that we want to define as a crossing probability.

Let $d$ be large but small in proportion to $|z|$, and for simplicity take $z=$ $(x, 0)$ with $x>0$. Since we shall be applying the notions of conformal invariance we treat $z$ as a point in the complex plane. To estimate the probability $P(z, d)$ that 0 is occupied and that the cluster containing it meets the disk of radius $d$ about $z$, we apply a conformal transformation $\phi$ that takes this disk to the exterior of a circle of radius $R$, and has derivative equal to 1 at the point 0 . (It is natural to assume that conformal invariance is applicable to events involving points only if the scale at the points is preserved.) Since the scale is preserved at 0 , conformal invariance suggests that

$$
P(z, d) \sim P(R) \sim R^{-1 / \rho} .
$$

At this level of argument, it is not worthwhile to search for the precise formula for $\phi$. The approximation

$$
\phi: w \rightarrow \frac{x w}{x-w}
$$

is sufficient. It takes the origin to the origin, and the circle of radius $d$ about $x$ to the circle with center on the real line that contains both $-x(x+d) / d$ and $x(x-d) / d$. Thus $x^{2} / d$ is a fair approximation to $R$, and

$$
P(x, d) \sim\left(\frac{x^{2}}{d}\right)^{-5 / 48}
$$

Thus

$$
P(x, d) \sim \tau(0, z) / d^{-5 / 48} .
$$

Now choose two large numbers $d_{1}$ and $d_{2}$, small in proportion to $x$, and consider the probability $P\left(z, d_{1}, d_{2}\right)$ that there is a cluster that meets both the disk of radius $d_{1}$ about 0 and the disk of radius $d_{2}$ about $z$. Symmetry suggests that

$$
P\left(z, d_{1}, d_{2}\right) \sim\left(\frac{x^{2}}{d_{1} d_{2}}\right)^{-5 / 48}
$$

On the other hand the mapping (2.5c) takes the region outside the two disks about 0 and $z$ to the annular region between two circles of radii about $d_{1}$ and $x^{2} / d_{2}$ and with centers close to 0 . We conclude that the probability of a 
crossing from one side to another of an annulus with center 0 and radii $r_{1}<r_{2}$ is approximately

$$
\left(\frac{r_{2}}{r_{1}}\right)^{-5 / 48}
$$

This relation is confirmed by numerical simulations that we do not present and that were much less systematic than those of Part Three. It yields a definition of $\eta$ in terms of the crossing probabilities for an annulus.
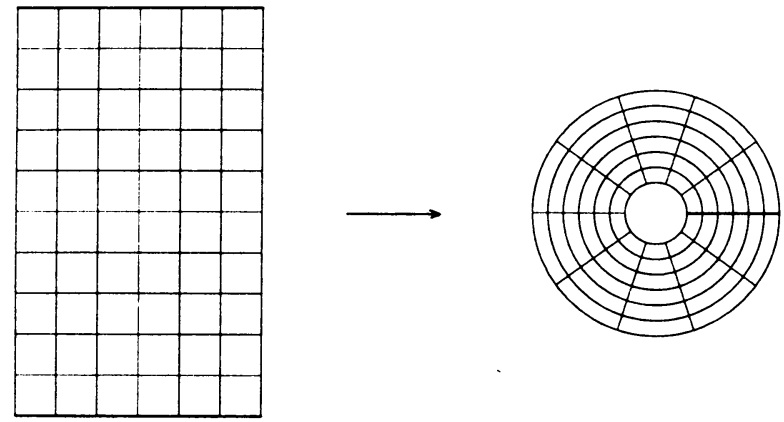

Figure 2.5. The map used to define the exponent $\eta$ in the finite models. (The radial scale of the second drawing is logarithmic.)

In the numerical studies ([L2]) of finite models, no attempt has been made to determine an approximate value for $\eta$. The procedure that might be used is clear. Suppose that, as in $\S 2.3$, we define the finite model by a decomposition of the sides of a square into $l$ intervals of equal length. The map $\boldsymbol{\theta}$ was defined by juxtaposing four such squares into a $2 \times 2$ array. If $m$ and $n$ are two integers, we can also juxtapose $m n$ squares to form an $m \times n$ array. The definition of $\boldsymbol{\theta}$ can be extended to give crossing probabilities between intervals of length $1 / l$ in the resulting rectangle of base $n$ and height $m$.

The function

$$
\exp \left(\frac{2 \pi(z+1)}{m}\right)
$$

takes the rectangle of base $\{0, n\}$ and side $\{0, i m\}$ to the annulus of radii $\exp (2 \pi / m)$ and $\exp (2 \pi(n+1) / m)$. Provided $m>1$ the annulus is thus represented as the glueing of $m n$ conformally distorted squares, as in Figure 2.5 , and the definition of $\Theta$ could be mimicked to define at a finite level the probability of crossing an annulus.

2.6. Conformally invariant fields and percolation. In response to Aizenman's suggestion of conformal invariance Cardy [C4] proposed, on the basis of the theory of conformally invariant fields, a formula for the horizontal crossing probability $\pi_{h}(r)$ on rectangles of aspect ratio $r$. In other words, if one takes $E$ to be defined by a rectangular curve $R$ of width $a$ and height $b$ such that $r=a / b$ and by opposing horizontal sides $\alpha, \beta$ with no excluded crossings, then a formula for $\pi\left(E, M_{0}\right)$ can be obtained that is confirmed by the numerical results of [U] and of $\S 3.2$ below. The coincidence of the predicted values with those found by simulation is the strongest evidence yet for conformal invariance. We stress nonetheless that the conformal invariance for events $E$ other 
than those defined by a single pair of intervals is not yet, even conjecturally, a consequence of the theory of conformally invariant fields.

To give two intervals on the simple closed curve $C$ is to give four points $z_{1}, z_{2}, z_{3}$, and $z_{4}$ in clockwise order. The first two are the endpoints of $\alpha$ and the last two the endpoints of $\beta$. There is a conformal (holomorphic) map of the interior of $C$ to the unit disk that takes $C$ to the circumference and $z_{1}, z_{2}, z_{3}, z_{4}$ to four points $w_{1}, w_{2}, w_{3}, w_{4}$. The map is not uniquely determined, for it can be followed by any conformal automorphism of the disk. Only the cross-ratio

$$
\frac{\left(w_{4}-w_{3}\right)\left(w_{2}-w_{1}\right)}{\left(w_{3}-w_{1}\right)\left(w_{4}-w_{2}\right)}
$$

is uniquely determined. It is a real number between 0 and 1 . Thus we may choose, and it is convenient to do so, the four points $w_{i}$ so that $w_{1}=w_{0}=$ $\exp \left(i \theta_{0}\right), w_{2}=\bar{w}_{0}, w_{3}=-w_{0}$, and $w_{4}=-\bar{w}_{0}$. Then the cross-ratio is $\sin ^{2}\left(\theta_{0}\right)$. Observe that $0 \leq \theta_{0} \leq \frac{\pi}{2}$ or $\pi \leq \theta_{0} \leq \frac{3 \pi}{2}$. Interchanging $\alpha$ and $\beta$ if necessary, we usually assume the first alternative.

If $E$ is the event defined by the rectangle $R, \alpha$, and $\beta$, then Cardy's formula for $\pi\left(E, M_{0}\right)$ is

$$
\pi\left(E, M_{0}\right)=\frac{3 \Gamma\left(\frac{2}{3}\right)}{\Gamma\left(\frac{1}{3}\right)^{2}} \sin ^{\frac{2}{3}}\left(\theta_{0}\right){ }_{2} F_{1}\left(\frac{1}{3}, \frac{2}{3}, \frac{4}{3}, \sin ^{2}\left(\theta_{0}\right)\right) .
$$

This is a function that equals 0 when $\theta_{0}=0$ and 1 when $\theta_{0}=\frac{\pi}{2}$, as it should.

In this paragraph we review the essential ideas of the derivation, which is not rigorous. Although the lattice models of statistical mechanics, their scaling limits, and conformally invariant field theories are objects that can be introduced in strictly mathematical terms, they arise, as we saw in $\S 2.2$, in a physical context rich in experience and inspiration whose sources of insight are unfamiliar to the mathematician, and of difficult access, so that, intimidated and sometimes at sea, he hesitates to apply his usual criteria. Our presentation of the ideas leading to Cardy's formula (2.6a) suffers from the attendant ambivalence; the authors have not all persuaded themselves that they fully comprehend to what extent the arguments are formal, inspired by the physical and historical connotations of the symbols, and to what extent they involve precisely defined mathematical entities. As stressed in the introduction, this section is not necessary to the understanding of Part Three.

In planar lattice models of statistical mechanics such as the Ising model a state $s$, before passage to the bulk limit, is described by its values at the sites of the lattice that lie in some large square. The interaction between the various points determines the energy $H(s)$ of the state, and its Boltzmann weight $\exp (-\beta H(s))$. The constant $\beta$, in essence the inverse temperature, may for our purposes be taken equal to 1 . The very important partition function is

$$
Z(\beta)=\sum_{s} \exp (-\beta H(s))
$$

It is used in particular to normalize the Boltzmann weights and thereby define a measure on the set of states,

$$
\mu(s)=\frac{\exp (-\beta H(s))}{Z(\beta)} .
$$


The natural functions of which to take expectations $E(f)$ are those that depend on the values $s(P)$ of the state at a finite number of points. For such a function one can expect that $E(f)$ continues to exist in the bulk limit.

The passage from the probabilistic concepts of statistical mechanics to a field theory can be presented rigorously as an analogue of that from a one-parameter semigroup to the associated infinitesimal generator ([GJ]); in practice, however, it is a much more adaptable and unconstrained mechanism.

For percolation, the procedure, quite apart from questions of the existence or nature of limits, does not appear promising. A state $s$ is determined by the occupied sites; the others are unoccupied. If their number is $N(s)$ then

$$
H(s)=\{-\ln p+\ln (1-p)\} N(s)
$$

and the Boltzmann weight is

$$
\exp (-H(s))=\left(\frac{p}{1-p}\right)^{N(s)}
$$

The value of the partition function is $(1-p)^{-N}$ if $N$ is the total number of sites in the square, and the probability of $s$ is $p^{N(s)}(1-p)^{N-N(s)}$.

These are the probabilities familiar from percolation, in which the value of the states at the sites are independent of each other. Thus if $f_{P}$ is a function of states given by

$$
f_{P}(s)=f(s(P)),
$$

the function $f$ being a function on the set of possible values, then for $r$ sites different from each other

$$
E\left(f_{P_{1}} f_{P_{2}} \ldots f_{P_{r}}\right)=E\left(f_{P_{1}}\right) E\left(f_{P_{2}}\right) \ldots E\left(f_{P_{r}}\right)=E(f)^{r} .
$$

Passing formally to operators and to limits, we see that

$$
E\left(f_{P_{1}} f_{P_{2}} \ldots f_{P_{r}}\right)=\left\langle\left|\phi\left(P_{1}\right) \phi\left(P_{2}\right) \ldots \phi\left(P_{r}\right)\right|\right\rangle,
$$

if $\phi(P)=\phi$ is constant and simply equal to a scalar $E(f)$ operating on a space of dimension one. Such trivial operators will not help in finding a formula for $\eta_{h}$, but these considerations do suggest that the central charge $c$ is 0 for percolation.

The statistical mechanics of lattices in a half space, or any bounded region, has, however, features that differentiate it from the theory in the full space. Boundary conditions have a much stronger effect; so familiar uniqueness theorems for Gibbs states and correlation functions need no longer apply. The consequences may continue to manifest themselves in the scaling limit. Cardy had pointed out in [C1] that at criticality and in two dimensions the limit could continue to exhibit conformal invariance, although of a somewhat different nature than for the scaling limit of bulk theories. In [C2] and [C3] he examined the effect of modification of the boundary conditions at the surface on the correlation functions in the interior.

From the principles [BPZ] that prescribe the behavior of conformally invariant fields in the full plane, we cite two. The first, a global principle, is that, if $P$ is treated as a complex parameter $z$, the correlation functions

$$
\left\langle\left|\phi\left(P_{1}\right) \ldots \phi\left(P_{r}\right)\right|\right\rangle
$$

may be treated as analytic functions of $z_{1}, \ldots, z_{r}$ and of their complex conjugates $\bar{z}_{1}, \ldots, \bar{z}_{r}$ and as such transform in a prescribed way under holomorphic 
(and antiholomorphic) maps $w(z)$. The simplest relation appears for the fields called primary:

$$
\left\langle\left|\phi\left(z_{1}, \bar{z}_{1}\right) \ldots \phi\left(z_{r}, \bar{z}_{r}\right)\right|\right\rangle=\prod w^{\prime}\left(z_{i}\right)^{h_{i}} \bar{w}^{\prime}\left(\bar{z}_{i}\right)^{\bar{h}_{i}}\left\langle\left|\phi\left(w_{1}, \bar{w}_{1}\right) \ldots \phi\left(w_{r}, \bar{w}_{r}\right)\right|\right\rangle,
$$

where the $h_{i}$ and $\bar{h}_{i}$ are known as the conformal dimensions of the field $\phi_{i}\left(z_{i}, \bar{z}_{i}\right)$.

At each point $P=z$, we may consider the algebras of formal holomorphic and antiholomorphic vector fields defined in a complement of the point (more precisely central extensions, the Virasora algebras, of these two algebras). The second principle is that there is an action of these algebras on the spaces underlying the fields and on the fields of operators themselves. There are conditions of compatibility, but they are subtle.

Conformally invariant fields are introduced in order to describe the asymptotic behavior at large differences of correlation functions of field theories, either on a lattice or in the continuum, in the sense of [GJ], so that it is perhaps ingenuous to expect them to have the same kind of operator significance. They are defined by Laurent series in which the individual coefficients are meaningful objects; thus they can be integrated against a limited class of functions on appropriate curves surrounding the point under consideration. Since the theory is conformally invariant, one could pass to the Riemann sphere and take this curve to be the image of a straight line in the plane, thereby recovering more familiar objects, but this seems to us to do violence to the spirit of the subject.

In two dimensions a simple choice of half space is the upper half-plane, with the real axis as boundary, and in this context there are further principles [C3, pp. 584-585] that are not at all obvious, at least to us; indeed we are not at all confident that we have adequately comprehended Cardy's views. The principles need nevertheless to be stressed.

A first, patent, principle is that the relevant algebra is not the sum of the holomorphic and antiholomorphic algebras, but the diagonal algebra contained therein, for the real axis, as the boundary of the region, must be left invariant.

Secondly, there are two pertinent classes of boundary conditions with quite different properties, those that are translation invariant, thus homogeneous on the entire boundary, and those that are homogeneous on both sides of 0 (so that scaling is still meaningful) but differ from one side to the other.

For those that are homogeneous on the entire line, it appears not unreasonable to expect that the underlying spaces are direct sums of irreducible representations of the Virasoro algebra, although the possibility of imposing different homogeneous boundary conditions may entail a rich variety of sectors in these sums. We do not yet understand to what extent other representations than the trivial one are necessary for percolation with homogeneous boundary conditions (whatever these might be!). For a boundary condition with a transition at 0 the representations of the Virasoro algebra need not be irreducible. The vacuum associated to these boundary conditions is not translation invariant, and thus is not annihilated by $L_{-1}$.

It appears that the sector (or theory, or, more concretely, the underlying Hilbert space - it is a matter of terminology) defined by such boundary conditions can be obtained from the full homogeneous sector by applying an operator $\phi=\phi(0)$. Once we have identified the boundary operators, and persuaded ourselves of the conformal invariance, so that the operators depend on a parameter 
$z$, they can be used to insert boundary conditions at several points.

We have already remarked that the first representation of the Virasoro algebra that appears in the study of percolation is the trivial representation. Useless though it appears to be for the study of correlation functions, it did yield immediately the value 0 for the central charge $c$.

The primary boundary operator $\phi(0)$ acting on the vacuum |\rangle will yield the vacuum $\phi(0)|\rangle$ associated to the boundary conditions, and $\phi(0)|\rangle$ will be the highest-weight vector of a representation of the Virasoro algebra. According to the results of [RW1, RW2] and authors there cited, an example of a representation that has the trivial representation as a quotient but for which the highest-weight vector is not translation invariant is obtained by dividing the Verma module with parameters $c=0$ and $h=0$ by the submodule with parameters $c=0$ and $h=2$. Since this is the submodule generated by the null vector corresponding to the root

$$
h_{1,2}=\frac{((m+1)-2 m)^{2}-1}{4 m(m+1)}=0, \quad m=2,
$$

of the Kac determinant formula, Cardy writes $\phi_{1,2}$ rather than $\phi$.

This argument, however, is far from satisfactory, for we have not even been precise about the nature of the boundary conditions. Cardy's argument draws on more sources. In particular, it exploits a common, but entirely factitious, device for introducing boundary conditions into percolation by treating it as a degenerate case of the $q$-state Potts model. The device has the additional advantage that the crossing probabilities appear as correlation functions.

Recall [W] that the Potts model is a lattice model, in which there are $q \geq 1$ possible values $\sigma$ for a state at each site of a square lattice. The hamiltonian is

$$
H(\sigma)=\sum_{x, y} 1-\delta_{\sigma_{x}, \sigma_{y}}
$$

The sum runs over all pairs of nearest neighbors inside a large square laid over the lattice. Observe that the extra term 1 does not affect the Boltzmann weights. In contrast to percolation, when $q>1$ there is a genuine energy of interaction.

Let $\mathfrak{B}$ be the set of nearest-neighbor bonds. The partition function for free boundary conditions is obtained by summing

$$
\exp (-\beta H(\sigma))=\prod_{\{x, y\} \in \mathcal{B}}\left(e^{-\beta}+\left(1-e^{-\beta}\right) \delta_{\sigma_{x}, \sigma_{y}}\right)
$$

Setting $p=1-e^{-\beta}$ we may write this as

$$
(1-p)^{d}
$$

with $d$ equal to the number of bonds joining two sites with $\sigma_{x} \neq \sigma_{y}$. We may also write it as a sum over the subsets $\mathfrak{x}$ of $\mathfrak{B}$,

$$
\sum p^{B(\mathfrak{x})}(1-p)^{B-B(\mathfrak{x})} \prod_{\{x, y\} \in \mathfrak{x}} \delta_{\sigma_{x}, \sigma_{y}} .
$$

The integer $B$ is the total number of bonds and $B(\mathfrak{x})$ the number of bonds in $\mathfrak{x}$. 
Each subset $\mathfrak{x}$ of $\mathfrak{B}$ decomposes the set $\mathfrak{S}$ of sites into clusters, two points lying in the same cluster if they can be joined by a sequence of bonds in $\mathfrak{x}$. The product

$$
\prod_{\{x, y\} \in \mathfrak{x}} \delta_{\sigma_{x}, \sigma_{y}}
$$

is 0 or 1 , and is 1 if and only if $\sigma$ is constant on each cluster. We write $\mathfrak{x} \rightarrow \mathfrak{A}$ if $\mathfrak{A}$ is the family of clusters determined by $\mathfrak{x}$. The clusters in $\mathfrak{A}$ are denoted $A_{1}, \ldots, A_{r}$. The integer $r$ is equal to the number $N(\mathfrak{A})$ of clusters in $\mathfrak{A}$. The sum (2.6b) is also equal to a sum over all possible decompositions into clusters,

$$
\sum_{\mathfrak{A}} \sum_{\mathfrak{x} \rightarrow \mathfrak{A}} p^{B(\mathfrak{x})}(1-p)^{B-B(\mathfrak{x})} \prod_{i} \prod_{\{x, y\} \in A_{i}} \delta_{\sigma_{x}, \sigma_{y}} .
$$

Taking the sum over all states, we find, as in [E1, §2.2], that the partition function with free boundary conditions is equal to

$$
Z_{f}=\sum_{\mathfrak{A}} \sum_{\mathfrak{x} \rightarrow \mathfrak{A}} p^{B(\mathfrak{x})}(1-p)^{B-B(\mathfrak{x})} q^{N(\mathfrak{A})}
$$

To examine the effect of boundary conditions we consider a rectangle, imposing boundary conditions on the left and right sides but leaving the top and bottom free. Suppose we demand that $\sigma$ take only the value $\alpha$ on the left side and only the value $\beta$ on the right side. Then the partition function is $Z_{\alpha, \beta}$ and it is obtained from $(2.6 \mathrm{c})$ on replacing $N(\mathfrak{A})$ by the number $N^{\prime}(\mathfrak{A})$ of clusters that do not intersect the left or right sides. Moreover, if $\alpha \neq \beta$ then all families of clusters with a member that meets the left and right sides are excluded from the sum. Consequently the difference

$$
Z_{\alpha, \alpha}-Z_{\alpha, \beta} \quad \alpha \neq \beta,
$$

is equal to the sum of the expression

$$
\sum_{\mathfrak{x} \rightarrow \mathfrak{A}} p^{B(\mathfrak{x})}(1-p)^{B-B(\mathfrak{x})} q^{N^{\prime}(\mathfrak{A})}
$$

over those families of clusters that do contain a member that intersects both sides of the square.

In particular, setting formally $q=1$ we obtain the sum over all subsets $\mathfrak{x}$ of the set of bonds that admit a horizontal crossing of

$$
p^{B(\mathfrak{x})}(1-p)^{B-B(\mathfrak{x})}
$$

When $p$ is the critical probability for bond percolation this is the probability of a horizontal crossing, thus in essence $\pi_{h}$.

We have progressed in two ways. First of all, the crossing probability $\pi_{h}$ has been identified as a difference of partition functions, and thus, as we shall see, as a difference of correlations. Secondly there is a free parameter $q$ and with a little bit of courage, we can transfer results for $q>1$ to $q=1$. That the condition $\alpha \neq \beta$ can not be realized for $q=1$ will trouble only the fainthearted, for it will never explicitly enter our manipulations of (2.6d).

What is relevant in (2.6d) is that the expression is a linear combination of partition functions with boundary conditions that change at four points, the four corners of the rectangle, from fixed to free. Although the transition from 
partition functions to correlation functions appears to be more a matter of intuition than of logic, persuasive only after much experience with the passage from lattice models to operators, it does appear rather explicitly in Cardy's reflections [C $3, \mathrm{pp} .584-585]$ for the case of a transition from a homogeneous condition $\sigma(x)=\alpha$ to the condition $\sigma(x)=\alpha$ for $x<0$ and $\sigma(x)=\beta$ for $x>0$. The corresponding operator is denoted somewhat informally as $\phi_{\alpha, \beta}$ or $\phi_{\alpha, \beta}(0)$. We suppress from the notation that there is also a jump in the boundary conditions at $\infty$, and of course admit the possibility that $\alpha$ signifies a free boundary condition, as well as a definite value of the spin or other variable.

In the context of conformally invariant theories it is possible to use the transformation $w=\ln z$ to replace the upper half-plane, with the point 0 on the boundary distinguished, by the strip $0 \leq \Im w \leq \pi$. Translation-invariant boundary conditions are transferred to boundary conditions equal on both sides, and translation invariant with respect to the strip. Boundary conditions with a jump are transferred to boundary conditions different on both sides of the strip, but translation invariant with respect to it. Experience with limits of standard lattice models, above all the Ising model, makes clear that calculating partition functions and correlation functions, or rather their limits, on such strips with boundary conditions at $\Im w=0$ and $\Im w=\pi$ is above all a matter of calculating the eigenvector $v_{\alpha, \beta}$ associated to the smallest eigenvalue of the transfer matrix associated to these conditions. If |\rangle is the eigenvector associated to equal homogeneous boundary conditions and $\phi_{\alpha, \beta}$ is an operator taking |\rangle to the eigenvector $v_{\alpha, \beta}$ then a correlation function

$$
\left\langle\left|\phi_{1} \ldots \phi_{r}\right|\right\rangle
$$

is replaced by

$$
\left\langle\left|\phi_{\alpha, \beta}^{*} \phi_{1} \ldots \phi_{r} \phi_{\alpha, \beta}\right|\right\rangle=\left\langle\left|\phi_{\beta, \alpha} \phi_{1} \ldots \phi_{r} \phi_{\alpha, \beta}\right|\right\rangle \text {. }
$$

However we have implicitly allowed a jump in the boundary conditions at 0 and at $\infty$, so that, indicating the dependence of one of the operators on the point 0 and the other on the point $\infty$, this equation might be rewritten as

$$
\left\langle\left|\phi_{\beta, \alpha}(\infty) \phi_{1} \ldots \phi_{r} \phi_{\alpha, \beta}(0)\right|\right\rangle \text {. }
$$

Transforming back to the upper half plane, and allowing insertions of modifications at several, say four, points, one of which may be at infinity, we obtain, for $r=0$,

$$
\left\langle\left|\phi_{\alpha, \beta}\left(z_{1}\right) \phi_{\beta, \gamma}\left(z_{2}\right) \phi_{\gamma, \delta}\left(z_{3}\right) \phi_{\delta, \alpha}\left(z_{4}\right)\right|\right\rangle .
$$

If $r>0$ it is less clear where to insert the operators in (2.6e), but $r=0$ is the pertinent value of $r$ for this is the value for which (2.6e) is a partition function. Although the modification in the boundary values was taken to be from one prescribed value to another and not from a prescribed value to free boundary conditions, the same arguments are valid in both cases. It is the transition from free to fixed, $\phi_{f, \alpha}$, and from fixed to free, $\phi_{\alpha, f}$, that appear in (2.6d) because the one pair of sides on which the boundary conditions are fixed are separated by the other sides on which they are free.

Cardy [C1, C2, C3, C4] does not find the operators $\phi_{\alpha, f}$ directly. Rather he argues first (for $q>1$ but also by extrapolation for $q=1$ ) that the operator 
$\phi_{\alpha, \beta}$ associated to the transition from one fixed boundary condition to another, different, fixed condition is the primary field $\phi_{1,3}$, and then that the operatorproduct expansion of $\phi_{\alpha, f}(z) \phi_{f, \beta}(w)$, which would be

$$
\phi_{\alpha, f}(z) \phi_{f, \beta}(w) \sim \delta_{\alpha, \beta} \mathbf{1}+\phi_{\alpha, \beta}
$$

implies that

$$
\phi_{\alpha, f}=\phi_{1,2} \text {. }
$$

Since his final argument is somewhat more convincing for unitary theories than for non-unitary theories, it is again best to regard it as extending to $q=1$ by extrapolation.

The identification of the operators $\phi_{\alpha, \beta}$ appeals to experience with specific models that, like the operator-product expansion itself, may be unfamiliar to the mathematician; so we observe that the numbers $\pi(E, M)$ are, by their very definition, invariant under dilations of the data defining $E$. In particular, if $(2.6 \mathrm{~g})$ is to represent a probability of crossing between intervals defined by $z_{1}, z_{2}, z_{3}$, and $z_{4}$ then it must be homogeneous of degree 0 in the vector $\left(z_{1}, z_{2}, z_{3}, z_{4}\right)$. Since the operator $\phi_{\alpha, f}(z)=\phi_{f, \alpha}(z)$ is primary it is homogeneous of some degree $h$, and $h$ must be 0 .

Although, in principle, any positive real number $h$ is a possible degree of homogeneity, those that occur most commonly are those associated to reducible Verma modules, and these are given by the Kac formula, which at $c=0$ becomes

$$
h_{p, q}=\frac{1}{24}\left((3 p-2 q)^{2}-1\right),
$$

where $p$ and $q$ are positive integers. The simplest choices of $p$ and $q$ that give $h=0$ are $p=q=1$, which leads to the trivial representation, and $p=1$, $q=2$, that yield $\phi_{\alpha, f}=\phi_{1,2}$.

To complete the derivation of Cardy's formula, we use the ideas of [BPZ] as presented in $[\mathrm{SA}]$ to find the differential equation satisfied by $(2.6 \mathrm{~g})$. The null vector $v_{1,2}$ in the Verma module with parameter $c=1-6 / m(m+1)$ is

$$
\left(L_{-1}^{2}-\frac{1}{3}\left(4 h_{1,2}+2\right) L_{-2}\right)\left|h_{1,2}\right\rangle \text {. }
$$

where $\left|h_{1,2}\right\rangle$ is the highest weight vector of the Verma module. For $c=0$ and $m=2, h_{1,2}=0$. Moreover, according to formula (4.6.21) of [SA], to find the differential equations satisfied by $(2.6 \mathrm{~g})$ we replace $L_{-k}$ in $(2.6 \mathrm{~h})$ by

$$
\mathscr{L}_{-k}=-\sum_{i=1}^{3} \frac{1}{\left(z_{i}-z_{4}\right)^{k-1}} \partial_{i},
$$

an expression that the relation $h_{1,2}=0$ has made much simpler than it would otherwise be.

The translation invariance permits the replacement of

$$
-\sum_{i=1}^{3} \partial_{i}
$$

by $\partial_{4}$, so that the differential equation satisfied by $(2.6 \mathrm{~g})$ is

$$
\left(\partial_{4}^{2}+\frac{2}{3}\left(\frac{1}{z_{3}-z_{4}} \partial_{3}+\frac{1}{z_{2}-z_{4}} \partial_{2}+\frac{1}{z_{1}-z_{4}} \partial_{1}\right)\right)\langle\ldots\rangle=0 .
$$


If we set

$$
z=\frac{\left(z_{1}-z_{2}\right)\left(z_{3}-z_{4}\right)}{\left(z_{1}-z_{3}\right)\left(z_{2}-z_{4}\right)}
$$

then conformal invariance implies that $(2.6 \mathrm{~g})$ is a function $g$ of $z$ alone.

With a little effort we infer from (2.6i) that $g$ satisfies the equation

$$
z(1-z)^{2} g^{\prime \prime}+2 z(z-1) g^{\prime}+\frac{2}{3} g^{\prime}-\frac{2}{3} z^{2} g^{\prime}=0
$$

or upon simplification

$$
z(1-z) g^{\prime \prime}+\frac{2}{3}(1-2 z) g^{\prime}=0 .
$$

This is a degenerate hypergeometric equation with two solutions $g \equiv 1$ and

$$
g(z)=z^{\frac{1}{3}}{ }_{2} F_{1}\left(\frac{1}{3}, \frac{2}{3}, \frac{4}{3} ; z\right) .
$$

To determine which linear combination of these two solutions is pertinent to our problem, we take $z_{1}, z_{2}, z_{3}$, and $z_{4}$ in decreasing order to be the images of the four vertices of the rectangle in clockwise order, starting with the lower left corner. If $r$ is the aspect ratio of the rectangle then $z \rightarrow 0$ when $r \rightarrow \infty$ and $z \rightarrow 1$ when $r \rightarrow 0$. Thus the solution yielding the crossing probability $\pi_{h}\left(R_{r}, M_{0}\right)$ must be a constant times $(2.6 \mathrm{j})$. The identity

$$
\frac{3 \Gamma\left(\frac{2}{3}\right)}{\Gamma\left(\frac{1}{3}\right)^{2}} z^{\frac{1}{3}}{ }_{2} F_{1}\left(\frac{1}{3}, \frac{2}{3}, \frac{4}{3} ; z\right)=1-\frac{3 \Gamma\left(\frac{2}{3}\right)}{\Gamma\left(\frac{1}{3}\right)^{2}}(1-z)^{\frac{1}{3}}{ }_{2} F_{1}\left(\frac{1}{3}, \frac{2}{3}, \frac{4}{3} ; 1-z\right)
$$

implies that the constant must be

$$
\frac{3 \Gamma\left(\frac{2}{3}\right)}{\Gamma\left(\frac{1}{3}\right)^{2}}
$$

in order that the function have the correct behavior at $z=1$. This is the formula (2.6a) of Cardy in a different notation (and for the upper half-plane rather than the unit disk.)

\section{THE EXPERIMENTS}

3.1. Experimental procedure. In order to provide some evidence for the hypotheses of universality and conformal invariance, we performed several simulations. Although several artifices had to be used in the various cases, the basic method is the same throughout: $(i)$ draw the curve $C$ defining the event $E$ on the lattice, (ii) assign randomly to each site of the lattice lying inside the curve a state (open with probability $p_{c}$, closed with probability $\left.\left(1-p_{c}\right)\right)$ and (iii) check whether the various crossings defining the event $E$ exist or not. These three steps are repeated till the desired sample size is reached. The estimated value of $\pi(E)$, denoted $\hat{\pi}(E)$, is then the ratio of the number of configurations satisfying the conditions of $E$ to the sample size.

For the above experimental procedure, the statistical errors are the easiest to assess. The sample size for all our experiments was at least $10^{5}$, and very often larger. For an estimated value $\hat{\pi} \sim 0.5$, this leads to a statistical error of $\Delta \hat{\pi} \sim 3 \times 10^{-3}$. For the largest $\hat{\pi}$ measurable $(\sim 0.999)$ or the smallest 
( $\sim 0.001$ ), the error is $\sim 2 \times 10^{-4}$. (All statistical errors are taken to represent a $95 \%$ confidence interval.)

The systematic errors are of various origins. Probably the least important source is the random number generator. We used in most of the experiments the linear congruential generator $x_{i+1}=\left(a x_{i}+c\right) \bmod m$, with

$$
a=142412240584757, \quad c=11, \quad m=2^{48} .
$$

It is of maximal period $m$. We believe it to be satisfactory.

A second source is the "value" of the probability $p_{c}$ appearing in the statement of the theorem of $\S 2.1$. This critical value $p_{c}$ is a well-defined concept only for percolation phenomena on an infinite lattice. But all our simulations are carried on finite lattices! The solution to this difficulty calls for a compromise. Indeed, on the one hand, lattices have to be chosen large enough to give a good approximation of the infinite case. On the other hand, a larger lattice requires a better approximation for $p_{c}$. (Recall that the slope of $\pi_{h}$ around $p_{c}$ increases with the size of the lattice, as depicted in Figure 2.1c.) The most suitable approximation depends, as discussed in [Z] and [U], on the size of the lattice; one could even imagine that it is different for rectangles containing the same number of sites but with distinct aspect ratios $r$. All the experiments but one were conducted at $p_{c}=0.59273$ with the curves $C$ containing from 40,000 to 200,000 sites. The only experiment that used a different $p_{c}$ was a repetition of the principal experiment of [U] where we measured the universal functions $\eta_{h}, \eta_{v}$ and $\eta_{h v}$ to be defined below. As these data together with Cardy's prediction are to be used as yardsticks for the new experiments, we felt that measuring them on a larger lattice was appropriate. For that experiment on a larger lattice, we used $p_{c}=0.5927439$. The six first digits in $p_{c}$ were definitely necessary to achieve the desired precision. The results are discussed in paragraph 3.2.

Another important source of systematic errors is the convention of crossings on finite lattices. The curve $C$ is to be drawn in the plane containing the lattice. We chose to define a crossing from the interval $\alpha$ to $\beta$ on $C$ as starting from a site inside $C$ joined to a neighbor by a bond intersecting the image of $\alpha$ of the lattice. Similarly the crossing must end at a site inside $C$ such that one of the attached edges intersect the image of $\beta$. Note that we might well have defined the crossing as starting from an open site outside the curve $C$ with one attached edge intersecting the interval in question. Hence the convention used introduces a systematic error. Moreover, one can imagine easily that sliding rigidly a rectangle on a square lattice by a fraction of the mesh might add a whole line or column to the set of inner sites, thus changing the estimate $\hat{\pi}$. For reasons described in [U], the attendant error for rectangles is commensurate with $\frac{2}{n} \pi^{\prime}$ where $\pi^{\prime}$ stands for the derivative of $\pi$ with respect to the aspect ratio $r$ and $n$ is the linear dimension of the rectangle. For a square containing $200 \times 200$ sites, the error on $\hat{\pi}_{h}$ turns out to be $\sim 5 \times 10^{-3}$, larger than the statistical error introduced by a sample of 100,000 configurations. We were on the whole content if the results obtained by simulation were consistent with those predicted by universality and conformal invariance within five parts in one thousand.

For the final experiments on conformal invariance, it grew slightly larger than one part in one hundred. This is not surprising in view of further specific sources 
of systematic errors, due to penetrating angles, branch points, and unbounded regions, that will be discussed as they arise. It does nevertheless make further experiments desirable.

The events studied in [U] were defined by a rectangular curve $C$. We chose the collection of intervals in four different ways. First of all, $\alpha$ could be the left side of the rectangle and $\beta$ the right, which yielded the probability $\pi_{h}(M)$ of a horizontal crossing, or $\alpha$ the lower side and $\beta$ the upper, which yielded the probability $\pi_{v}(M)$ of a vertical crossing. We also studied the probability $\pi_{h v}(M)$ of simultaneous horizontal and vertical crossings. The difference $\pi_{h}(M)-\pi_{h v}(M)$ provides an example of an event with a horizontal crossing but no vertical one. In the notation of $\S 2.3$, the intervals $\alpha, \delta, \beta, \gamma$ are then the left, upper, right and lower sides, respectively. For a little variety the probability $\pi_{d}(M)$ of a crossing from the upper half of the left side to the right half of the bottom side was also studied. In these functions there is an implicit variable $r$, the aspect ratio of the rectangle, that we took to be the quotient of the length of the horizontal side by that of the vertical side. Taking $M$ to be $M_{0}$ we obtain, as explained in [U], four universal functions, $\eta_{h}=\pi_{h}\left(M_{0}\right)$, $\eta_{v}=\pi_{v}\left(M_{0}\right), \eta_{h v}=\pi_{h v}\left(M_{0}\right)$ and $\eta_{d}=\pi_{d}\left(M_{0}\right)$ of $r$. The probabilities of similar events will be measured in some of the following experiments.

If the hypothesis of universality holds, the functions $\eta_{h}(r)$ and $\eta_{v}(r)$ are not independent. This can be seen by the following duality argument. We draw a rectangle on a triangular lattice. There will be a horizontal crossing (on open sites) if and only if there is no vertical crossing on closed sites. This is consistent with the theorem of $\S 2.1$ only if $p_{c}=\frac{1}{2}$ for this model (denoted $M$ ) and then

$$
\pi_{h}(r, M)+\pi_{v}(r, M)=1
$$

for all $r$. Of course, the argument could have been made for any closed curve $C$, disjoint intervals $\alpha$ and $\beta$, and the two disjoint intervals $\delta$ and $\gamma$ of their complement. The relation would then be

$$
\pi_{\alpha \leftrightarrow \beta}+\pi_{\delta \leftrightarrow \gamma}=1
$$

where $\pi_{\alpha \leftrightarrow \beta}$ stands for the probability of a crossing from $\alpha$ to $\beta$. Universality then implies that this relation holds for any model. This is a handy test of simulations. Observe that, for the model $M_{0}$, the complementarity of horizontal crossings on open sites and of vertical crossings on closed sites does not hold for individual configurations. Every experiment measuring simultaneously $\pi_{h}(M)$ and $\pi_{v}(M)$ on other models $M$, such as $M_{0}$, for which this complementarity does not hold for direct reasons serves as a check on universality.

In the tables, the results of the experiments are presented together with either Cardy's prediction when it is applicable or by values for rectangles inferred by interpolation from the experimental results of the next section. Cardy's prediction will be denoted by $\pi^{c f t}$ for conformal field theory and the estimated values for rectangles, as well as values calculated from them using interpolation, by $\hat{\pi}^{\square}$.

3.2. Experimental verification of Cardy's formula. The goal of the first experiment is twofold: to verify again Cardy's prediction for the function $\pi_{h}(r)$. on $M_{0}$ and to obtain values of $\pi_{h v}\left(r, M_{0}\right)$ suitable for comparison in other experiments. A similar experiment was performed and reported in [U] before Cardy 
proposed his formula. Here we increase the number of sites inside the rectangles from the approximately 40,000 used in [U] to $1,000,000$ and the sample size to $10^{6}$ configurations. For the reasons explained above, $p_{c}$ was taken to be 0.5927439 . (This value compares well with the conclusions of $Z$ iff $[Z]$ that came to our attention after the first version of the paper was prepared.) The results, tabulated in Table 3.2, are a replacement for those of Table III of [U] and are suitable for calculating the values $\hat{\pi}_{h v}^{\square}$ by interpolation.

If one uses $s=\ln r$ instead of $r$, the function $\ln \left(\pi_{h} /\left(1-\pi_{h}\right)\right)$ is odd because of the relation $\pi_{h}+\pi_{v}=1$. The estimated values of this function (dots) are plotted against Cardy's prediction (continuous line). The values $\hat{\pi}_{v}$ were used for $r<1$. For $s \sim 0$., the measured values of $\ln \left(\pi_{h} /\left(1-\pi_{h}\right)\right.$ carry a statistical error of $\sim 4 \times 10^{-3}$ and for $|s| \sim 2$. this error increases to $2 \times 10^{-2}$. These errors are too small to be indicated in Figure 3.2, being in the worst situation approximately the size of the dots themselves.

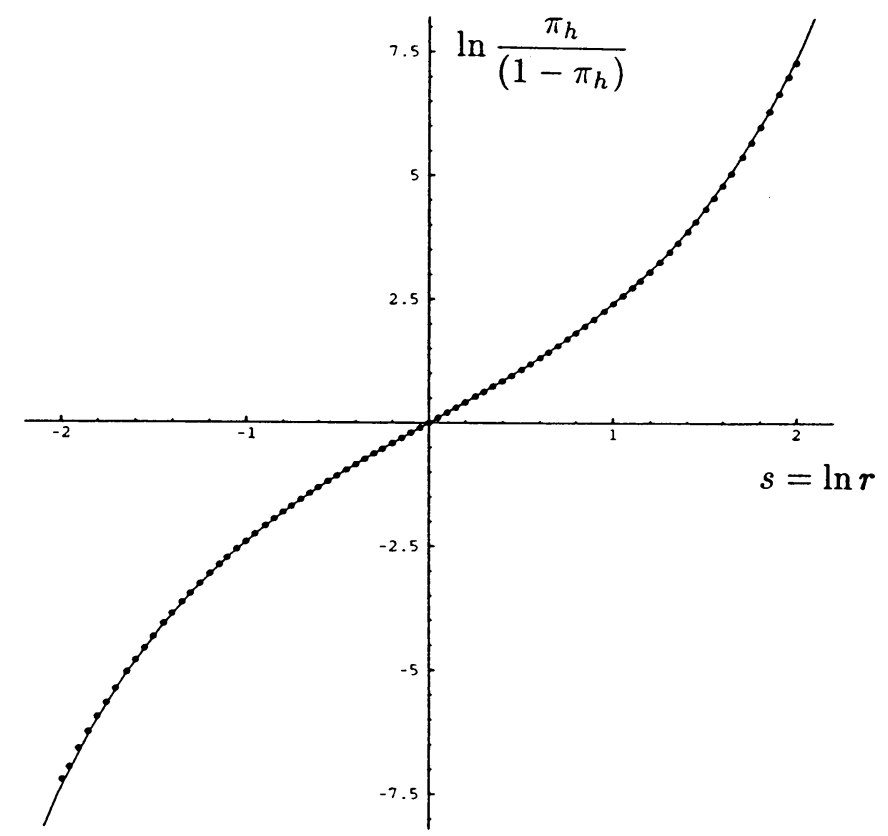

FIGURE 3.2. Comparison of 81 measured values of $\ln \pi_{h} /\left(1-\pi_{h}\right)$ (dots) with Cardy's prediction (curve).

A glance at Table 3.2 shows that the difference $\hat{\pi}_{h}-\pi_{h}^{c f t}$ is positive for all $r$. Though it is always $\leq 6 \times 10^{-4}$ and smaller than the statistical error, a systematic error is suggested. Ziff ([Z]) gave an heuristic description of the "good" value of $p_{c}$ for the square using finite-size scaling arguments and simulations. For other curves $C$, including rectangles of large or small aspect ratio, the "best" values of $p_{c}$ for finite lattices are not available. It may be that the values of $p_{c}$ are different for the measurements of $\pi_{h}$ and of $\pi_{v}$ on the same rectangle, and that this is the source of the error. Observe finally that, with this size of lattice, a change of a few units in the sixth digit of $p_{c}$ could account for the discrepancies between $\pi_{h}^{c f t}$ and $\hat{\pi}_{h}$. Despite the systematic error, the agreement is remarkable and we shall compare the results of the following experiments with $\pi^{c f t}$ instead of $\hat{\pi}^{\square}$ when the former is applicable. 
TABLE 3.2. $\hat{\pi}_{h}, \hat{\pi}_{v}, \hat{\pi}_{h v}$ on $M_{0}$ for various values of the aspect ratio $r$.

\begin{tabular}{|c|c|c|c|c|c|c|c|}
\hline width & height & $r$ & $r^{-1}$ & $\pi_{h}^{c f t}$ & $\hat{\pi}_{h}$ & $\hat{\pi}_{v}$ & $\hat{\pi}_{h v}$ \\
\hline 1000 & 1000 & 1.000 & 1.0000 & 0.5000 & 0.5001 & 0.4999 & 0.3223 \\
\hline 1025 & 975 & 1.051 & 0.9512 & 0.4740 & 0.4743 & 0.5257 & 0.3211 \\
\hline 1050 & 950 & 1.105 & 0.9048 & 0.4480 & 0.4484 & 0.5516 & 0.3180 \\
\hline 1080 & 930 & 1.161 & 0.8611 & 0.4226 & 0.4230 & 0.5768 & 0.3127 \\
\hline 1105 & 905 & 1.221 & 0.8190 & 0.3970 & 0.3974 & 0.6026 & 0.3055 \\
\hline 1135 & 880 & 1.290 & 0.7753 & 0.3695 & 0.3696 & 0.6301 & 0.2950 \\
\hline 1160 & 860 & 1.349 & 0.7414 & 0.3473 & 0.3475 & 0.6522 & 0.2854 \\
\hline 1190 & 840 & 1.417 & 0.7059 & 0.3235 & 0.3236 & 0.6762 & 0.2733 \\
\hline 1220 & 820 & 1.488 & 0.6721 & 0.3003 & 0.3004 & 0.6994 & 0.2600 \\
\hline 1250 & 800 & 1.562 & 0.6400 & 0.2777 & 0.2779 & 0.7217 & 0.2458 \\
\hline 1285 & 780 & 1.647 & 0.6070 & 0.2541 & 0.2543 & 0.7453 & 0.2297 \\
\hline 1315 & 760 & 1.730 & 0.5779 & 0.2330 & 0.2333 & 0.7666 & 0.2144 \\
\hline 1350 & 740 & 1.824 & 0.5481 & 0.2111 & 0.2117 & 0.7883 & 0.1976 \\
\hline 1385 & 725 & 1.910 & 0.5235 & 0.1929 & 0.1935 & 0.8065 & 0.1826 \\
\hline 1420 & 705 & 2.014 & 0.4965 & 0.1731 & 0.1736 & 0.8265 & 0.1657 \\
\hline 1455 & 685 & 2.124 & 0.4708 & 0.1542 & 0.1546 & 0.8450 & 0.1490 \\
\hline 1490 & 670 & 2.224 & 0.4497 & 0.1389 & 0.1392 & 0.8606 & 0.1351 \\
\hline 1530 & 655 & 2.336 & 0.4281 & 0.1236 & 0.1239 & 0.8761 & 0.1210 \\
\hline 1570 & 640 & 2.453 & 0.4076 & 0.1093 & 0.1096 & 0.8905 & 0.1077 \\
\hline 1610 & 620 & 2.597 & 0.3851 & 0.09402 & 0.09424 & 0.9055 & 0.09299 \\
\hline 1650 & 605 & 2.727 & 0.3667 & 0.08201 & 0.08212 & 0.9176 & 0.08132 \\
\hline 1690 & 590 & 2.864 & 0.3491 & 0.07104 & 0.07120 & 0.9286 & 0.07065 \\
\hline 1735 & 575 & 3.017 & 0.3314 & 0.06053 & 0.06082 & 0.9390 & 0.06047 \\
\hline 1775 & 565 & 3.142 & 0.3183 & 0.05314 & 0.05332 & 0.9463 & 0.05309 \\
\hline 1820 & 550 & 3.309 & 0.3022 & 0.04459 & 0.04478 & 0.9549 & 0.04465 \\
\hline 1870 & 535 & 3.495 & 0.2861 & 0.03669 & 0.03689 & 0.9629 & 0.03682 \\
\hline 1915 & 520 & 3.683 & 0.2715 & 0.03016 & 0.03037 & 0.9695 & 0.03031 \\
\hline 1965 & 510 & 3.853 & 0.2595 & 0.02523 & 0.02542 & 0.9744 & 0.02539 \\
\hline 2015 & 495 & 4.071 & 0.2457 & 0.02009 & 0.02033 & 0.9796 & 0.02032 \\
\hline 2065 & 485 & 4.258 & 0.2349 & 0.01651 & 0.01670 & 0.9832 & 0.01669 \\
\hline 2115 & 470 & 4.500 & 0.2222 & 0.01281 & 0.01286 & 0.9869 & 0.01285 \\
\hline 2170 & 460 & 4.717 & 0.2120 & 0.01020 & 0.01022 & 0.9895 & 0.01022 \\
\hline 2225 & 450 & 4.944 & 0.2022 & 0.00805 & 0.00807 & 0.9918 & 0.00807 \\
\hline 2280 & 440 & 5.182 & 0.1930 & 0.00627 & 0.00634 & 0.9936 & 0.00634 \\
\hline 2340 & 425 & 5.506 & 0.1816 & 0.00447 & 0.00453 & 0.9954 & 0.00453 \\
\hline 2400 & 415 & 5.783 & 0.1729 & 0.00334 & 0.00340 & 0.9966 & 0.00340 \\
\hline 2460 & 405 & 6.074 & 0.1646 & 0.00247 & 0.00258 & 0.9975 & 0.00258 \\
\hline 2520 & 395 & 6.380 & 0.1567 & 0.00179 & 0.00190 & 0.9982 & 0.00190 \\
\hline 2585 & 385 & 6.714 & 0.1489 & 0.00126 & 0.00135 & 0.9987 & 0.00135 \\
\hline 2650 & 375 & 7.067 & 0.1415 & 0.00087 & 0.00093 & 0.9991 & 0.00093 \\
\hline 2720 & 370 & 7.351 & 0.1360 & 0.00065 & 0.00072 & 0.9993 & 0.00072 \\
\hline
\end{tabular}


3.3. Parallelograms. This second experiment investigates the hypothesis of conformal invariance for simple curves $C$, namely parallelograms. The model is again $M_{0}$. One obvious consequence of the conformal hypothesis is that the relative orientation of the square lattice and of the parallelogram $C$ should be irrelevant in the measurement of $\pi(E)$. This rotational symmetry is to be contrasted with the obvious finite group of symmetries of $M_{0}$. Stronger consequences of full conformal invariance can be tested by comparing the simulations on a parallelogram that is not rectangular with the simulations on a rectangle.

Any parallelogram can be obtained from the square $P_{0}$ by applying an element $g$ of $G L(2, \mathbb{R})$. If we take the square to be that defined by the points $(0,0),(1,0),(1,1),(0,1)$ and $g$ to be

$$
\left(\begin{array}{ll}
a & b \\
c & d
\end{array}\right)
$$

then $P=g P_{0}$ is given by $(0,0),(a, c),(a+b, c+d),(b, d)$. We stress that $g$ is not conformal.

If, for example, $\pi_{h}(P, M)$ is the probability of a horizontal crossing for large dilations of $P$ with respect to the model $M$ then

$$
\pi_{h}\left(P, M_{0}\right)=\pi_{h}\left(P_{0}, g^{-1} M_{0}\right)
$$

may be thought of as a coordinate of the model $g^{-1} M_{0}$, that defined by a horizontal crossing of dilations of $P_{0}$. With our identification of the image of $\psi$ with the upper half-plane, the point $g^{-1} M_{0}$ corresponds to

$$
\frac{a i+c}{b i+d}=\frac{a-c i}{b-d i} \text {. }
$$

If we identify in the natural way $\mathbb{R}^{2}$ with the complex numbers, this is (unfortunately) the complex conjugate of the quotient of the vertical by the horizontal side of $P$.

To verify conformal invariance by simulation, we use the conformal map $\varphi$ sending the unit circle to $P$, and $w_{0}$, whose value will depend on $P$, to $(0,0)$, the point $-\bar{w}_{0}$ to $(a, c)$, the point $-w_{0}$ to $(a+b, c+d)$, and $\bar{w}_{0}$ to $(b, d)$. There will be exactly one rectangle $P_{1}$ with vertices $(0,0),(h, 0),(h, v)$, $(0, v)$ that is conformally equivalent to $P$ together with its vertices, or to the unit circle together with the specified four vertices. With $r=h / v$, conformal invariance entails the relations

$$
\pi_{h}\left(P, M_{0}\right)=\eta_{h}(r) ; \quad \pi_{v}\left(P, M_{0}\right)=\eta_{v}(r) ; \quad \pi_{h v}\left(P, M_{0}\right)=\eta_{h v}(r) .
$$

Thus, in effect, given a parallelogram we find, in two steps a conformal map that takes its interior to the interior of a rectangle and takes vertices to vertices and sides to sides. Since the intermediate curve is a circle with four distinguished points, we have a choice. We can compare $\hat{\pi}_{h}\left(P, M_{0}\right)$ and $\hat{\pi}_{v}\left(P, M_{0}\right)$ with Cardy's predictions, or we can compare them with the values for $\pi_{h}\left(P_{1}, M_{0}\right)$ and $\pi_{v}\left(P_{1}, M_{0}\right)$ interpolated from those given in Table 3.2 of the previous section. We prefer to compare with Cardy's predicted values. For $\hat{\pi}_{h v}\left(P, M_{0}\right)$ however, we have only the second alternative.

The values of $\pi_{d}\left(P, M_{0}\right)$ can also be predicted by Cardy's formula, but only after they are precisely defined. They can be defined as the probability of a 
crossing between the upper half of the left side of $P$ to the right half of the bottom side. If, on the other hand, $\alpha$ and $\beta$ are the images of the upper half of the left side of $P_{1}$ and the right half of the bottom side, they can also be defined as the probability of a crossing from $\alpha$ to $\beta$. Both definitions were used, according to the whim of the individual experimenter, and we shall distinguish them as the first and second definitions.

Although superfluous we provide in Figure 3.3 some curves in the upper halfplane on which conformal invariance implies that the three functions $\pi_{h}\left(P_{0}, M\right), \pi_{v}\left(P_{0}, M\right)$, and $\pi_{h v}\left(P_{0}, M\right)$, taken as functions of $z=\psi(M)$, are constant.

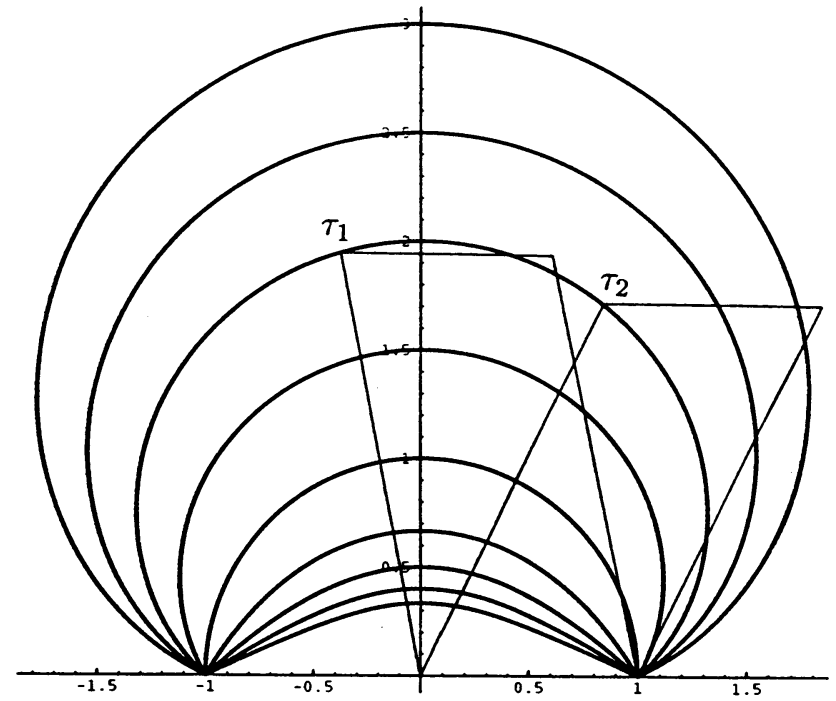

FIGURE 3.3. Two parallelograms with vertices $(0,1$, $\left.\tau_{1}+1, \tau_{1}\right)$ and $\left(0,1, \tau_{2}+1, \tau_{2}\right)$ will have the same $\pi_{h}$ if and only if $\tau_{1}$ and $\tau_{2}$ lie on the same curve.

To obtain these curves we employ the Schwarz-Christoffel transformation,

$$
\varphi: w \rightarrow \int_{0}^{w}\left(u^{2}-w_{0}^{2}\right)^{\alpha-1}\left(u^{2}-\bar{w}_{0}^{2}\right)^{-\alpha} d u=\frac{1}{w} \int_{0}^{1}\left(u^{2}-\epsilon_{1}^{2}\right)^{\alpha-1}\left(u^{2}-\epsilon_{2}^{2}\right)^{-\alpha} d u
$$

with

$$
\epsilon_{1}=\frac{w_{0}}{w}, \quad \epsilon_{2}=\frac{\bar{w}_{0}}{w}
$$

It maps the circle to a parallelogram with vertices, in clockwise order, $\varphi\left(w_{0}\right)$, $\varphi\left(\bar{w}_{0}\right), \varphi\left(-w_{0}\right), \varphi\left(-\bar{w}_{0}\right)$. The interior angle at the vertex $\varphi\left(w_{0}\right)$ is $\alpha \pi$. It does not matter that the parallelogram is not in standard position.

Fixing $w_{0}$ and letting $\alpha$ vary from 0 to 1 , we obtain one of the curves in Figure 3.3 as the collection of points

$$
z=\frac{\varphi\left(w_{0}\right)-\varphi\left(\bar{w}_{0}\right)}{\varphi\left(w_{0}\right)-\varphi\left(-\bar{w}_{0}\right)} .
$$

As parameters for a parallelogram, we can take $\alpha$ and $w_{0}$, or more conveniently $\alpha$ and the quotient $r$ of the lengths of the two sides. To conform with the notation of [U] we take $r=1 /|z|$. The data in Table 3.3 are from sixteen 
sets of experiments, corresponding to four values of $\alpha: 1 / 2,3 / 8,1 / 4,1 / 8$. In addition we chose four positions for the parallelogram, one in which a side was parallel to the imaginary axis (labelled as the case $\theta_{0}$ ), and then rotations of this clockwise through angles $\theta_{1}=\pi / 12, \theta_{2}=\pi / 6$, and $\theta_{3}=\pi / 4$. Conformal invariance entails, as observed, rotational invariance, so that the rotation of the parallelogram should not affect the result. In each experiment there were eleven values for $r$, chosen so that the values of $\hat{\pi}_{h}$ were about the same in each experiment and covered a representative range. The data are divided into four sets, each corresponding to a given value of $\alpha$. In each set the values of the various crossing probabilities for different values of $\theta$ are listed in adjacent columns to facilitate visual comparison. The probabilities $\pi_{d}$ are those given by the first definition. The sample size was 100,000 . The lengths of the sides were then chosen so that there would be about 40000 sites inside the parallelogram. As we observed in [U] and §3.1, with this number of sites an error of about five parts in a thousand is to be expected. There appears to be a systematic error of this order in the data. For example the experimental values corresponding to the true value $\pi^{c f t}=.5$ are largely less than .5 . When the parallelogram is not a rectangle with sides parallel to the axes, the collection of sites within the parallelogram has an irregular boundary. We were not able to find a method for accounting systematically for the errors resulting from the anfractuosities.

The measurements $\hat{\pi}_{h}, \hat{\pi}_{v}, \hat{\pi}_{h v}$ and $\hat{\pi}_{d}$ for all values of the angle at the vertex $\alpha$, of the angle of rotation $\theta$ and of the ratio $r$ agree with the corresponding $\pi^{c f t}(E)$ or $\hat{\pi}_{h v}^{\square}$ within the statistical errors and limitations due to the finiteness of the lattices. Examining the rows at which $\pi^{c f t}(E)=.5$, we see that the worst discrepancies are .0045 for $\alpha=1 / 2, .0024$ for $\alpha=3 / 8, .0039$ for $\alpha=1 / 4$, and .0057 for $\alpha=1 / 8$. As $\alpha$ grows smaller, the parallelogram grows more skew, and the finite size of our lattices less and less tolerable. For a given number of lattice points and sufficiently small $\alpha$ the simulations no longer make any sense, but $\alpha=1 / 8$ yields acceptable results.

3.4. Striated models. By numerical simulation we showed in [U] that the four functions $\pi_{h}, \pi_{v}, \pi_{h v}$ and $\pi_{d}$ coincided for the six following models: percolation by sites and by bonds on square, triangular and hexagonal lattices. This gives some support to the hypothesis of universality. Because of the symmetry of these regular lattices, the matrix $g$ predicted by the hypothesis is diagonal in all six cases. This need not to be so, as the following example shows.

If we restrict ourselves to percolation by sites, it is pretty clear that, within the limits of experimental observation, the most general case can be obtained by choosing on the lattice $L=\mathbb{Z}^{2}$ probabilities $p(s)$ that depend on the position of $s$ modulo some sub-lattice $N L$, where $N$ is a very large integer. This is certainly convenient for simulations. In particular to obtain a model that does not yield a point on the imaginary axis, we can deliberately skew the usual model by insisting that the probabilities be close to 0 along some band athwart the lattice and otherwise, as far as conditions of periodicity permit, close to 1. This we call a striated model. The hypothesis of universality implies that any model $M$, and in particular any striated model, corresponds to a point in the upper half-plane, and that, once this point is known, all probabilities $\pi(E, M)$ can be calculated from those for percolation by sites on a square lattice. Since in the eyes of many of our colleagues, universality even in the form proposed in the hypothesis is a commonplace, widely accepted and well 
.

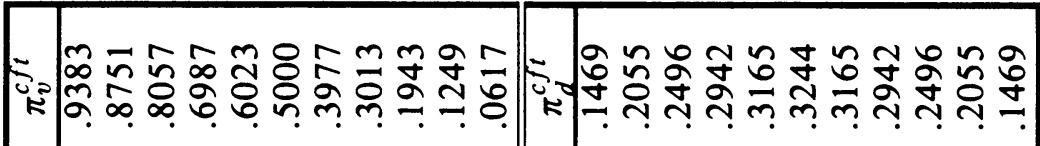

m-

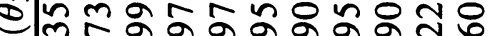

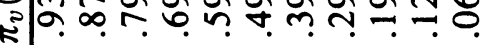

- ㅇำำำกำ

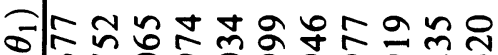

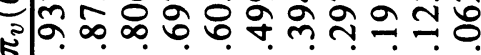

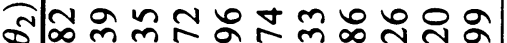

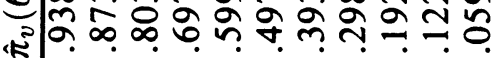

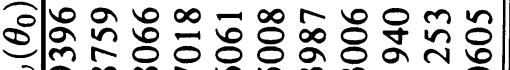

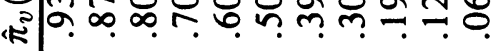

m무 $m \sim \infty n \sigma m \infty \forall N$

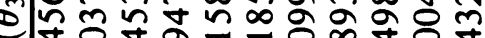

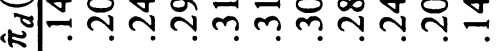

ণิ๓

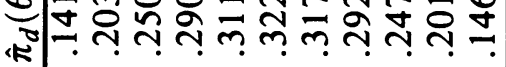

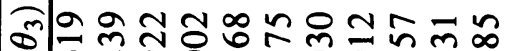

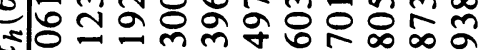

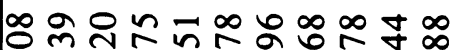

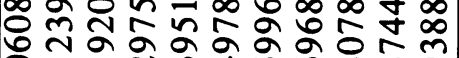

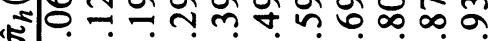

$\pi N \infty \infty N$ a

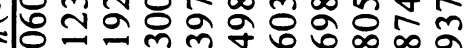
)

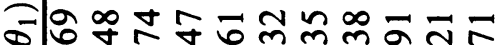

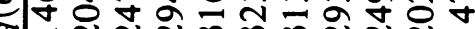

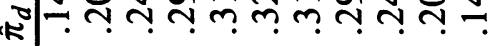

ఠิ

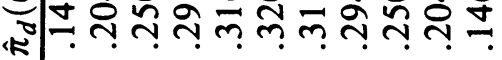

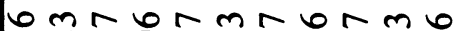

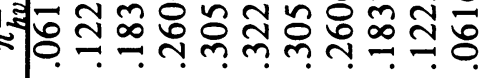

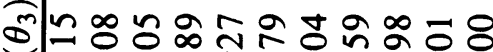

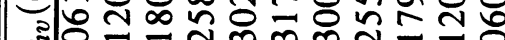
(र

कोก $n m$ a $\forall \infty$ o

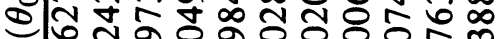

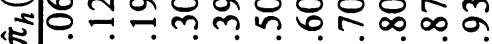

ฮิก 귕 $\sim$ ก 供

. $8 \infty \cdots \infty \infty$ 융 m

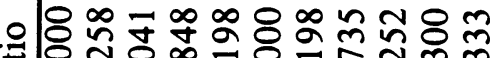

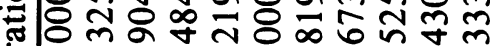
ल 


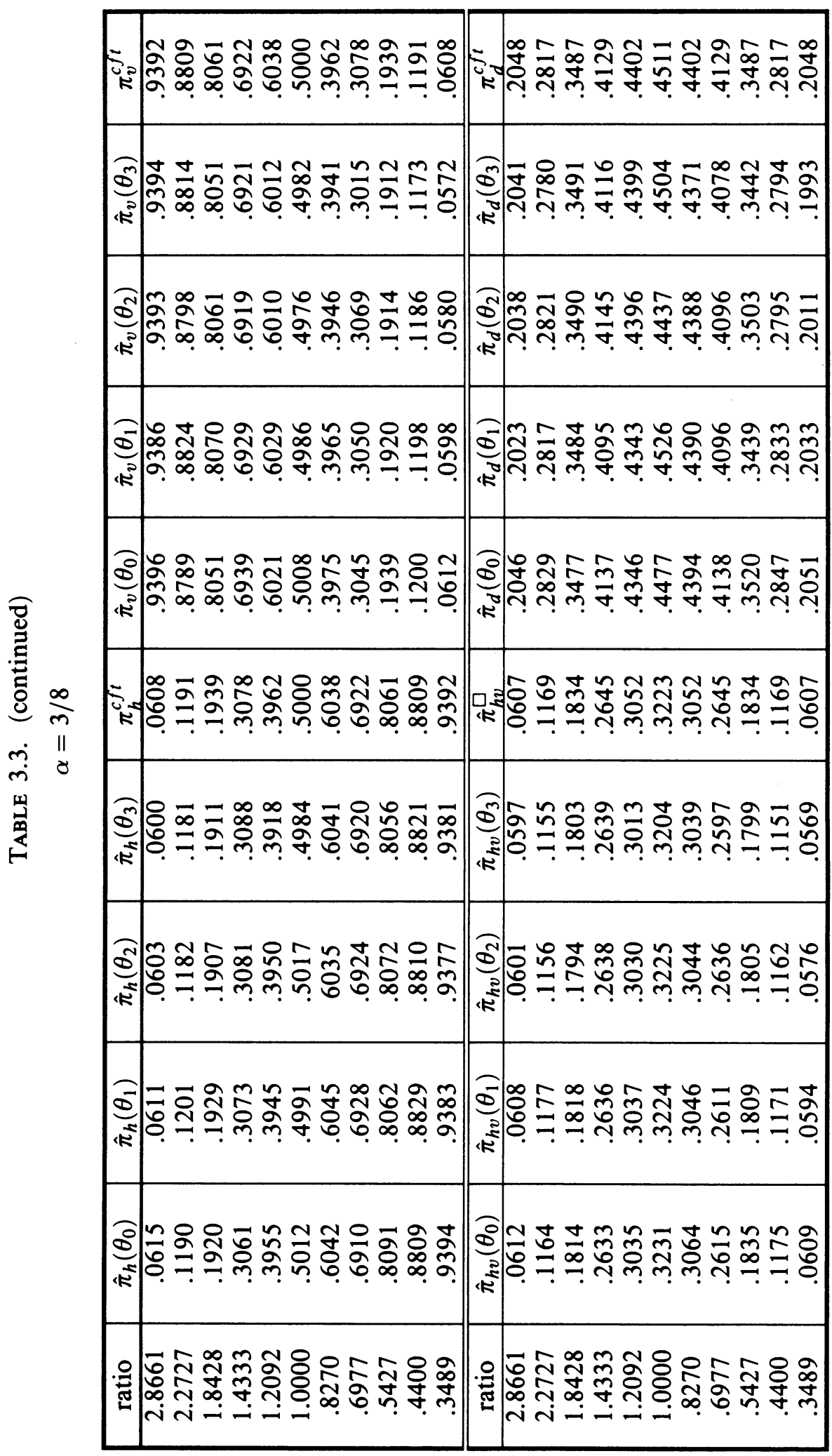




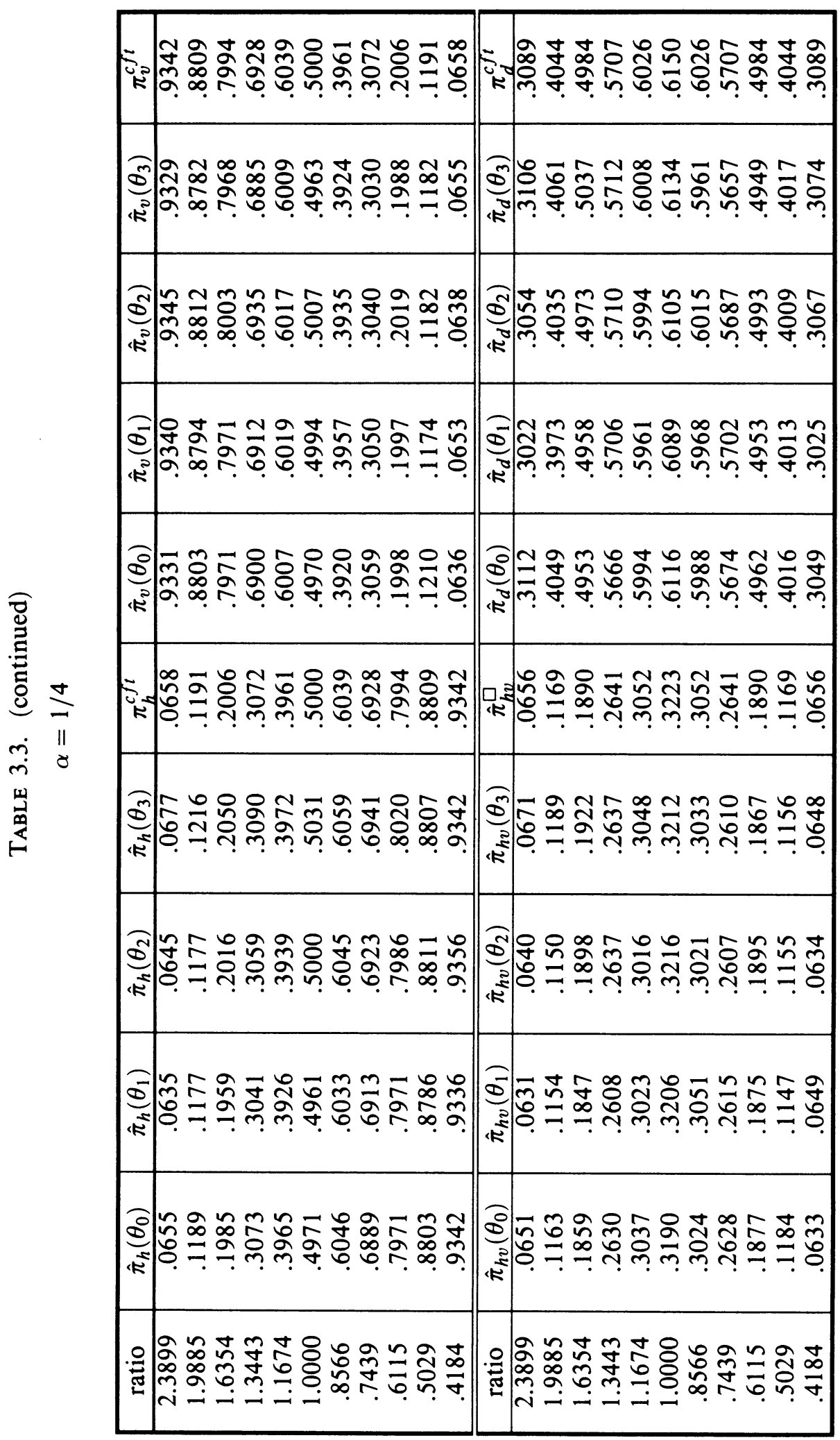




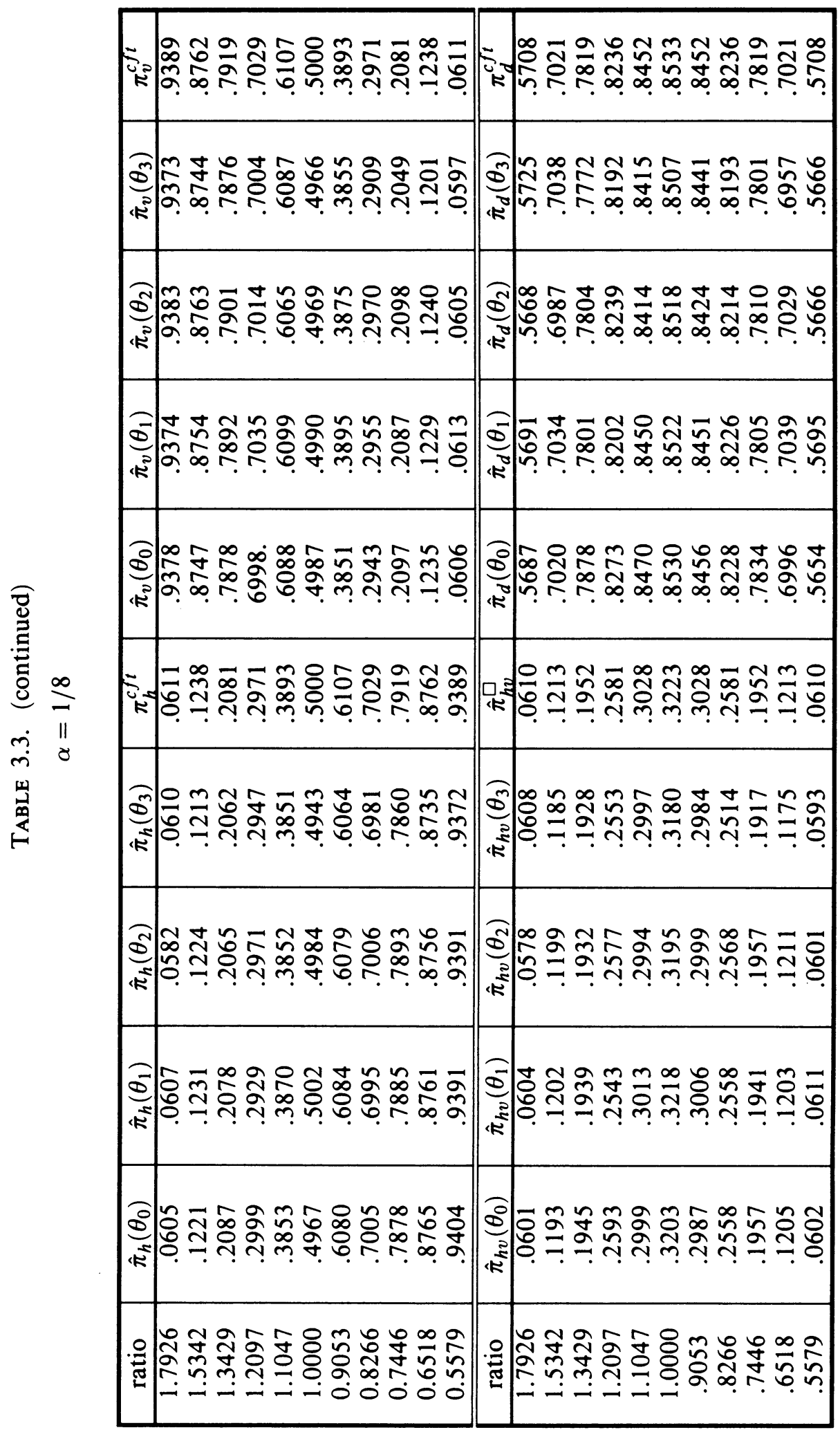


understood, we have confined ourselves here to the examination of a single example. It illustrates adequately the hypothesis, and the calculation of an approximation to the associated matrix $g$ is a useful exercise.

The band is constructed by periodicity from a rectangle with $6 \times 4$ sites, as in Figure 3.4. The points on the band are $(0,0),(1,0),(1,1),(2,1),(3,2)$, $(4,2),(4,3)$, and $(5,3)$. Thus $N=12$. (The bands are depicted by black squares in Figure 3.4.) All other points are off the bands. The probability $p_{1}$ on the bands is one-fifth the probability $p_{2}$ off the bands. Simulation and the technique of [U] yield a value $p_{2}=0.84928$ for the critical probability. The band forms an angle whose tangent is $2 / 3$ with the $\mathrm{x}$-axis and we can expect that the model corresponds to a dilation of one axis of the model $M_{0}$ followed by a rotation of approximately this angle, for what we have done is to hinder percolation perpendicular to the band, and to foster it parallel to the band. Thus the model presumably behaves like site-percolation on a rectangular lattice in which the basic rectangle has its long side parallel to the band.

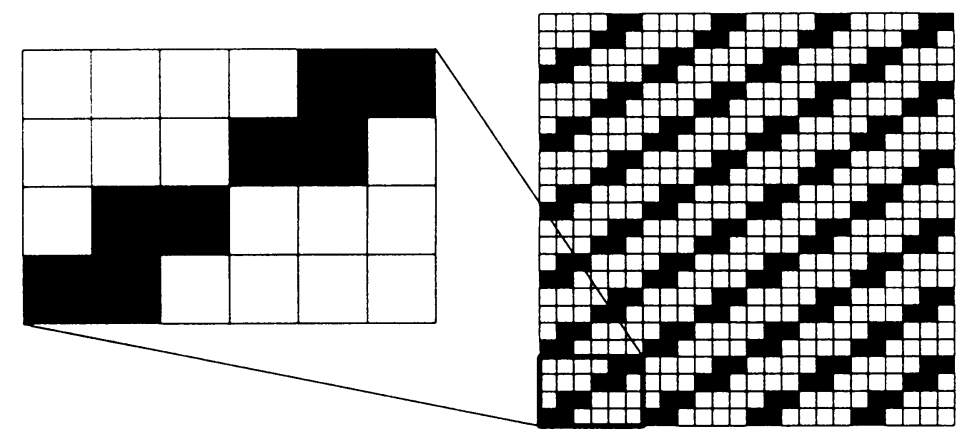

FIGURE 3.4. Definition of a striated model. Black sites (tiles) are open with probability $p_{1}$ and white ones with probability $p_{2}=5 p_{1}$.

According to the hypothesis of universality there will be a matrix $g$ such that

$$
\pi(E, M)=\pi\left(E, g M_{0}\right),
$$

for all events $E$. To calculate an approximation to $g$ we consider first the events defined by a horizontal crossing of a rectangle $R_{r}$ of aspect ratio $r$ with sides parallel to the coordinate axes. It is clear from our discussion of conformal invariance for parallelograms that, modulo the group of linear conformal transformations acting on the right and the group

$$
\left\{\left(\begin{array}{cc} 
\pm 1 & 0 \\
0 & \pm 1
\end{array}\right)\right\}
$$

acting on the left, there is at most one matrix $g$ such that

$$
\pi_{h}\left(R_{r}, M\right)=\pi_{h}\left(R_{r}, g M_{0}\right)=\pi_{f}\left(g^{-1} R_{r}, M_{0}\right)
$$

for all $r$, or even for two values of $r$. We repeat that the second equality is formal. We take, to be precise, $g^{-1}$ in the form

$$
\left(\begin{array}{cc}
a \sin \theta & 0 \\
-a \cos \theta & 1
\end{array}\right)
$$


with $0 \leq \theta \leq \pi$. The angle $\theta$ is the interior angle of the parallelograms $g^{-1} R_{r}$ and the right side of $(3.4 \mathrm{c})$ is calculated from Cardy's formula by the methods described in $\S 3.3$. The matrix $g$ itself is then a scalar multiple of

$$
\left(\begin{array}{cc}
1 & 0 \\
a \cos \theta & a \sin \theta
\end{array}\right)
$$

Equally useful is the relation

$$
\pi_{v}\left(R_{r}, M\right)=\pi_{v}\left(R_{r}, g M_{0}\right)=\pi_{v}\left(g^{-1} R_{r}, M_{0}\right) .
$$

Universality affirms that for the given striated model $M$ a matrix $g$ can be found such that (3.4a) is satisfied for all events $E$. The equations $(3.4 \mathrm{c})$ and (3.4d) are particular cases of (3.4a) that almost suffice to determine $g$. In Table $3.4 \mathrm{a}$ we give the left side of (3.4c) and (3.4d) for 41 values of $r$, or rather values obtained for the left side by simulation. The method of least squares was then used to find values of $a$ and $\theta$ that minimized the difference between the two sides of $(3.4 \mathrm{c})$ or $(3.4 \mathrm{~d})$, the right side being determined as described in $\S 3.3$. The values obtained are:

$$
\hat{a}=0.7538 \quad \hat{\theta}=0.2643 \pi .
$$

Let $\hat{g}$ be the associated matrix. For each value of $r$, the two parameters $\hat{a}$ and $\hat{\theta}$ are used to calculate, from Cardy's formula, the aspect ratios $r_{0}$ of the rectangles such that the numbers $\pi_{h}^{c f t}$ and $\pi_{v}^{c f t}$ appearing in the row of Table 3.4a labeled by $r$ are hypothetically equal by universality (assuming $\hat{g}$ is the matrix appearing in (3.4a)) to $\pi_{h}\left(R_{r_{0}}, M_{0}\right)$ and $\pi_{v}\left(R_{r_{0}}, M_{0}\right)$.

The ambiguity entailed by multiplication by the matrices $(3.4 \mathrm{~b})$ implies that the value $\hat{\theta}=\pi-0.2643 \pi$ is also possible; it leads to the same values of the right sides. Thus a second experiment is required to eliminate it.

Once estimates for $a$ and $\theta$ have been obtained, then for any parallelogram $P$ predicted values of $\pi_{h}(P, M), \pi_{v}(P, M)$, and of $\pi_{h v}(P, M)$ can be calculated from the right side of (3.4a) and Cardy's formula or by interpolation from Table 3.2, as in the section on parallelograms. As a first choice we took $P=\hat{g} R_{r_{0}}$, because, for example, we expect that

$$
\pi_{h}\left(g R_{r_{0}}, M\right)=\pi_{h}\left(R_{r_{0}}, M_{0}\right)=\eta_{h}\left(r_{0}\right) .
$$

One interior angle of the parallelogram $\hat{g} R_{r_{0}}$ would then be equal to $0.3502 \pi$.

The results appear in Table $3.4 \mathrm{~b}$, in which the variable $r_{0}$ is the free variable. Thus the coordinates of the vertices of the parallelograms on the striated lattice actually used are calculated from it. They are $(0,0),(0, b),(c, d)$ and $(c, b+d)$, where the integers $b, c, d$ assume the values in the table. The values of $r_{0}$ are given in the table; the ratio of the sides of the parallelogram $g R_{r_{0}}$ are then $r=\hat{B} r_{0}$ with

$$
\hat{B}=\frac{\sqrt{\left(1+\hat{a}^{2} \cos ^{2} \hat{\theta}\right)}}{\hat{a} \sin \hat{\theta}}=2.016 .
$$

It is clear from this table that of the two possibilities for $g$ modulo the group (3.4b) we have chosen the correct one, for otherwise there would be no agreement between the values obtained by simulation and the predicted values. 
TABLE 3.4a. Data for calculating the matrix $\hat{g}$ of the striated model.

\begin{tabular}{|c|c|c|c|c|c|}
\hline$r$ & $r_{0}$ & $\hat{\pi}_{h}$ & $\pi_{h}^{c f t}$ & $\hat{\pi}_{v}$ & $\pi_{v}^{c f t}$ \\
\hline & & & & & \\
0.6070 & 0.3873 & 0.9058 & 0.9045 & 0.0965 & 0.0955 \\
0.6400 & 0.4116 & 0.8885 & 0.8880 & 0.1146 & 0.1120 \\
0.6721 & 0.4356 & 0.8716 & 0.8711 & 0.1302 & 0.1289 \\
0.7059 & 0.4613 & 0.8546 & 0.8527 & 0.1492 & 0.1473 \\
0.7414 & 0.4887 & 0.8344 & 0.8327 & 0.1699 & 0.1673 \\
0.7753 & 0.5153 & 0.8147 & 0.8131 & 0.1881 & 0.1869 \\
0.8190 & 0.5502 & 0.7891 & 0.7874 & 0.2148 & 0.2126 \\
0.8611 & 0.5845 & 0.7641 & 0.7623 & 0.2388 & 0.2377 \\
0.9048 & 0.6206 & 0.7378 & 0.7361 & 0.2672 & 0.2639 \\
0.9512 & 0.6599 & 0.7114 & 0.7083 & 0.2933 & 0.2917 \\
1.000 & 0.7018 & 0.6801 & 0.6793 & 0.3228 & 0.3207 \\
1.051 & 0.7467 & 0.6521 & 0.6492 & 0.3534 & 0.3508 \\
1.105 & 0.7948 & 0.6210 & 0.6181 & 0.3832 & 0.3819 \\
1.161 & 0.8457 & 0.5893 & 0.5867 & 0.4145 & 0.4133 \\
1.221 & 0.9007 & 0.5562 & 0.5543 & 0.4458 & 0.4457 \\
1.290 & 0.9651 & 0.5188 & 0.5185 & 0.4816 & 0.4815 \\
1.349 & 1.021 & 0.4909 & 0.4891 & 0.5133 & 0.5109 \\
1.417 & 1.086 & 0.4594 & 0.4570 & 0.5455 & 0.5430 \\
1.488 & 1.155 & 0.4271 & 0.4252 & 0.5770 & 0.5748 \\
1.562 & 1.229 & 0.3957 & 0.3938 & 0.6086 & 0.6062 \\
1.647 & 1.313 & 0.3606 & 0.3607 & 0.6396 & 0.6393 \\
1.730 & 1.395 & 0.3302 & 0.3309 & 0.6692 & 0.6691 \\
1.824 & 1.490 & 0.3003 & 0.2998 & 0.7008 & 0.7002 \\
1.910 & 1.576 & 0.2750 & 0.2738 & 0.7277 & 0.7262 \\
2.014 & 1.681 & 0.2463 & 0.2453 & 0.7546 & 0.7547 \\
2.124 & 1.792 & 0.2204 & 0.2183 & 0.7836 & 0.7817 \\
2.224 & 1.894 & 0.1961 & 0.1963 & 0.8059 & 0.8037 \\
2.336 & 2.008 & 0.1758 & 0.1742 & 0.8277 & 0.8258 \\
2.453 & 2.127 & 0.1538 & 0.1538 & 0.8477 & 0.8462 \\
2.597 & 2.274 & 0.1326 & 0.1319 & 0.8695 & 0.8681 \\
2.727 & 2.407 & 0.1159 & 0.1147 & 0.8855 & 0.8853 \\
2.864 & 2.547 & 0.0990 & 0.0991 & 0.9010 & 0.9009 \\
3.017 & 2.703 & 0.0846 & 0.0842 & 0.9158 & 0.9159 \\
3.142 & 2.830 & 0.0744 & 0.0737 & 0.9269 & 0.9263 \\
3.309 & 3.001 & 0.0618 & 0.0616 & 0.9396 & 0.9384 \\
3.495 & 3.191 & 0.0512 & 0.0505 & 0.9497 & 0.9495 \\
3.683 & 3.382 & 0.0410 & 0.0413 & 0.9590 & 0.9587 \\
3.853 & 3.556 & 0.0346 & 0.0344 & 0.9661 & 0.9656 \\
4.071 & 3.778 & 0.0279 & 0.0273 & 0.9734 & 0.9727 \\
4.258 & 3.969 & 0.0230 & 0.0223 & 0.9780 & 0.9777 \\
4.500 & 4.217 & 0.0174 & 0.0172 & 0.9830 & 0.9828 \\
& & & & & \\
\hline & & & & &
\end{tabular}




\begin{tabular}{|c|c|}
\hline 足 & 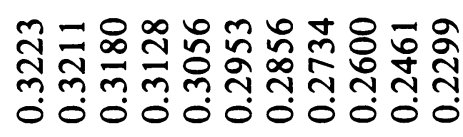 \\
\hline से & 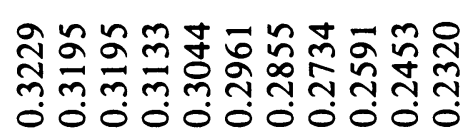 \\
\hline $\mathrm{S}^{\mathrm{N}}$ & 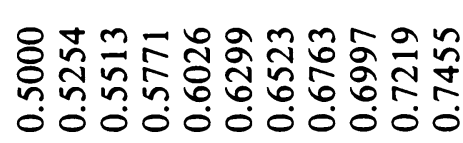 \\
\hline تم & 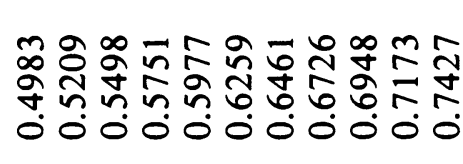 \\
\hline $\mathrm{S}^{\mathrm{v}}$ & 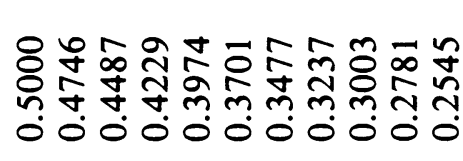 \\
\hline सE & 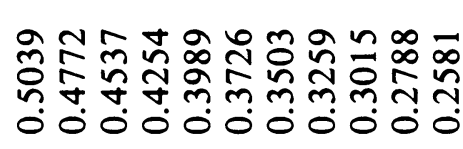 \\
\hline 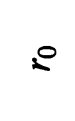 & 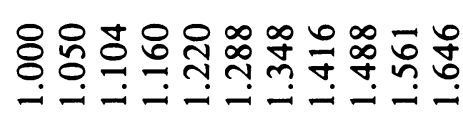 \\
\hline$\tau$ & 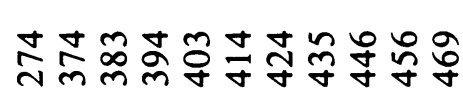 \\
\hline$u$ & 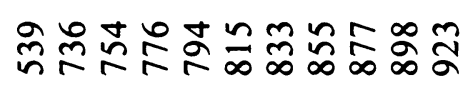 \\
\hline 0 & 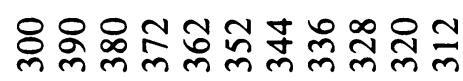 \\
\hline
\end{tabular}

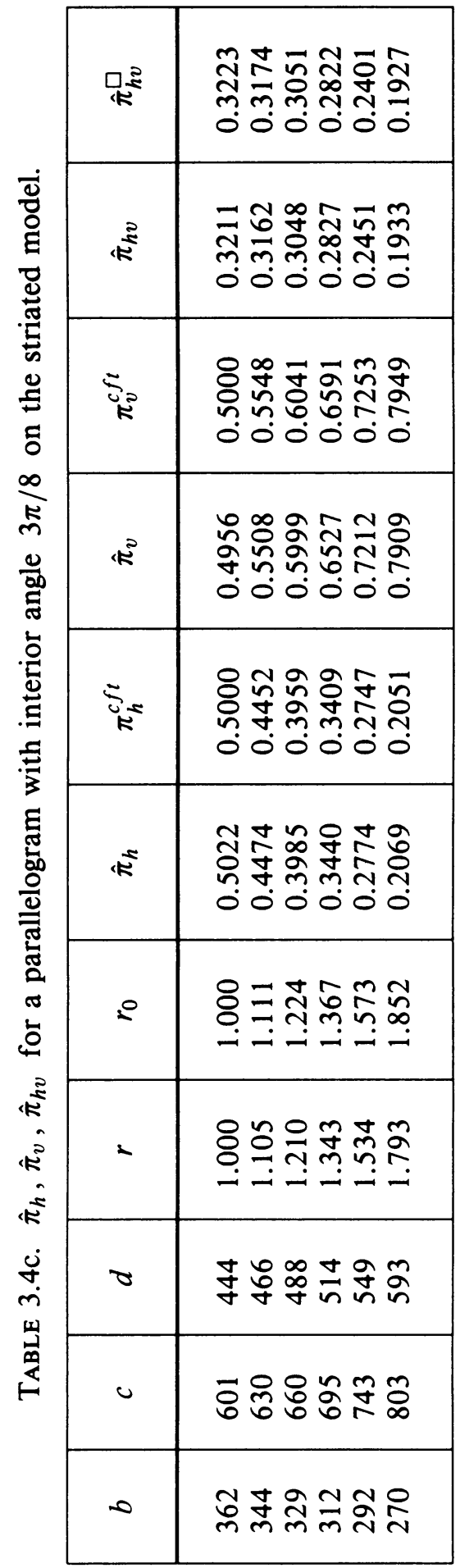


As a further verification we examined the probabilities like $\pi_{h}(\hat{g} P, M)$ for a parallelogram $P$ of interior angle $3 \pi / 8$, and with one pair of opposite sides vertical. One interior angle of the parallelogram $\hat{g} P$ is then very close to $0.2974 \pi$. The values $r$ in Table $3.4 \mathrm{c}$ are the ratios of sides of $\hat{g} P$. The values $r_{0}$ are the aspect ratios of rectangles conformally equivalent to $P$, and are used to calculate the predicted values given in Table 3.4c.

As for the previous experiment with parallelograms, a systematic error can be seen: for example, in both Table $3.4 \mathrm{~b}$ and $3.4 \mathrm{c}$ the value $\hat{\pi}_{h}$ is always larger than $\pi_{h}^{c f t}$. Still the discrepancy is in the third significant digit and comparable to the error due to the finiteness of the lattice (see $\S 3.1$ ); so the agreement is satisfactory. The only differences greater than .005 are those at row $(344,833,424)$ in Table $3.4 \mathrm{~b}$ and at row $(312,695,514)$ in Table $3.4 \mathrm{c}$, where differences of .0058 and .0064 are found. The anfractuosities at the boundary of $P$ may again have played a role.

3.5. Exterior domains. Once the notion of conformal invariance has appeared in a convincing manner in the study of percolation in simply-connected bounded planar regions, many other questions arise. First of all there is no reason to confine oneself to simply connected regions, nor, apart from experimental inconvenience, to bounded regions. Even the notion of crossing probability can be considerably extended.

Limitations on memory force the simulations to be confined to a bounded region, and when examining unbounded regions it is necessary either to devise an experiment that is not sensitive to the inevitable hole at infinity, or to estimate the error it causes. Moreover the boundaries of unbounded regions, such as the exterior of a convex polygon, usually have angles that penetrate the region, and these are the source of substantial errors in the simulation.

Examining percolation by bonds on a square lattice, we saw in [U] that an indeterminacy of the order of the lattice mesh led to an indeterminacy of about $1 / d$ in the crossing probabilities, if $d$ is the diameter of the finite lattice. This is to be expected by Cardy's formula, at least for $\pi_{h}$. A modification of the order of 1 in the endpoint $z_{i}$ of $\alpha$ or $\beta$ entails a change in $w_{i}$ and thus in the cross-ratio of about $1 / d$. If, however, $z_{i}$ were the vertex of a penetrating wedge with exterior angle $\alpha \pi$, then near $w_{i}$ the function $\varphi$ behaves like $\left(w-w_{i}\right)^{\alpha}$ and its inverse like $\left(z-z_{i}\right)^{1 / \alpha}$. Consequently, if for example $\alpha=1.5$, an indeterminacy of say .01 is magnified to one of $.01^{2 / 3} \sim .05$, and the data cease to be persuasive.

In order to avoid problems with penetrating angles, we have confined ourselves to experiments with circles. The obvious question is whether percolation in the interior of the circle is equivalent to percolation in the exterior, thus whether crossing probabilities are invariant under the map $z \rightarrow 1 / z$. Since this takes the bounded domain $|z| \leq 1$ to the unbounded domain $|z| \geq 1$, we are immediately confronted with the impossibility of treating all lattice points in the exterior domain.

Take a circle $C$ of radius 1 centred at the origin, and let $\alpha$ be the arc of $C$ from $\frac{3 \pi}{4}$ to $\frac{5 \pi}{4}$ and $\beta$ its reflection in the axis of ordinates. Conformal invariance implies that the probability of a crossing from $\alpha^{\prime}=A \alpha$ to $\beta^{\prime}=A \beta$ in the exterior of $C^{\prime}=A C$ should be close to .5 for $A$ large. Experiments can, however, only be carried out on finite lattices. We can take, for example, 
percolation inside the annulus formed by two circles, the inner one having radius $A$ and the outer a radius as large as time and the machines available allow, and estimate the probability within this annulus of a crossing from $\alpha^{\prime}$ to $\beta^{\prime}$. The results are disappointing. For an inner radius of 100 and an outer radius of 1000 the probability is about .431 . With the same outer radius and inner radii of 50 and 25 the probabilities become about .457 and .468 , in every case far short of the expected .5 , although the value is seen to improve with increasing ratio of the two radii. It is also clear, however, that to achieve an adequate value of the ratio and of the inner radius would put impossible demands on machine memory. Therefore it is necessary either to exploit methods of extrapolation or to devise other experiments to test conformal invariance under inversion.

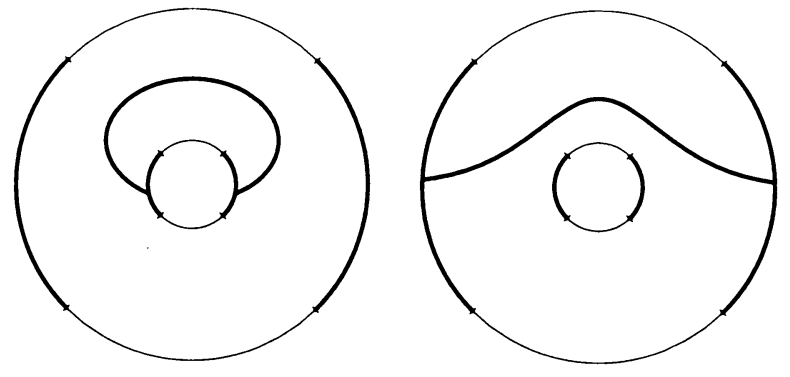

FIgURE 3.5a. Possible crossings for $\pi_{h}^{i n t}$ and $\pi_{h}^{e x t}$.

The most direct is to take the two concentric circles of radii $r_{1}<r_{2}$ and to divide each into four arcs of equal length symmetric about the axes. We consider only crossings within dilations of the annulus, and we introduce the probability $\pi_{h}^{\text {int }}$ of a crossing from the left interior arc to the right interior arc, as well as the probability $\pi_{h}^{e x t}$ of a crossing from the left exterior arc to the right exterior arc. (See Figure 3.5a.) The two probabilities $\pi_{v}^{i n t}$ and $\pi_{v}^{\text {ext }}$ are defined similarly. Conformal invariance under $z \rightarrow 1 / z$ implies that all four are equal in the limit of large $r_{1}$ and fixed $r_{2} / r_{1}$. We also introduce $\pi_{h v}^{i n t}$ and $\pi_{h v}^{e x t}$.

Table 3.5. $\hat{\pi}_{h}, \hat{\pi}_{v}, \hat{\pi}_{h v}$ for an annulus and a cylinder.

\begin{tabular}{|l|c|c|c|}
\hline & $\hat{\pi}_{h}$ & $\hat{\pi}_{v}$ & $\hat{\pi}_{h v}$ \\
\hline interior & .4316 & .4306 & .2539 \\
\hline exterior & .4356 & .4348 & .2586 \\
\hline cylinder & .4424 & .4399 & .2637 \\
\hline
\end{tabular}

The data for $r_{1}=100$ and $r_{2}=1000$ are given in Table 3.5. The sample size was 100,000 . As the difference between the values for the interior arcs and those for the exterior is in all three cases less than .005 , they confirm the conformal invariance. As a supplemental test of their reliability, we examined, again for the model $M_{0}$, crossings on a rectangle with horizontal side equal to $122 \sim A \ln \left(\frac{r_{1}}{r_{2}}\right)$ and vertical side equal to $332 \sim 2 A \pi, A=53$ but with periodic boundary conditions in the vertical direction. Let $\alpha, \beta, \gamma$ and $\delta$ be the intervals $[y=1, y=83],[y=167, y=249][y=84, y=166]$, and $[y=250, y=332]$ on the left side. Then we define $\pi_{h}^{l}$ as the probability of a 


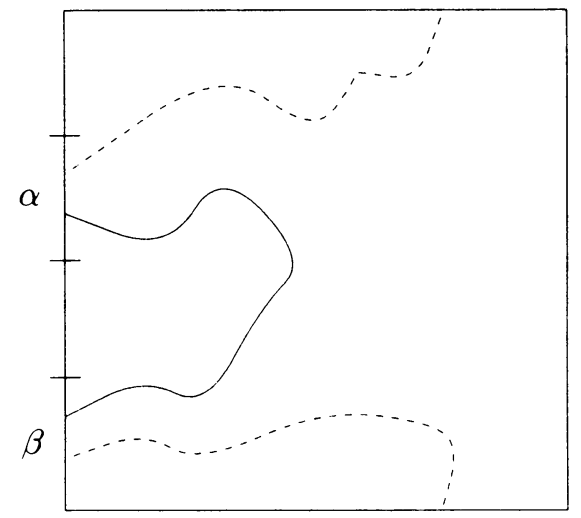

FIGURE 3.5b. Two possible paths for $\pi_{h}^{l}$.

crossing from $\alpha$ to $\beta$ in a vertically periodic geometry. Two possible paths are indicated in Figure 3.5b. We define the other probabilities, for example $\pi_{v}^{l}$, in a similar fashion.

If we extend the hypothesis of conformal invariance to assert that crossing probabilities on an annulus should be equal (for the model $M_{0}$ on which we have chosen to focus) to those on a conformally equivalent cylinder then, apart from the approximations inherent in the use of finite lattices, these crossing probabilities should be equal to the corresponding probabilities for crossings between the internal and external intervals of the annulus. The results are also included in Table 3.5 as line cylinder. The discrepancies are larger than .01 and therefore disappointing, but tolerable especially in view of the small inner radius $r_{1}$. Recall that a systematic error $\sim 5 \times 10^{-3}$ is to be expected on a square of $200 \times 200$ sites! (See $\S 3.1$.) In addition, a cylinder with the given dimensions is conformally equivalent to an annulus whose radii are in the ratio 10.06 .

In yet another test we examined the same probabilites for a vertical side equal to 240 and a horizontal side equal to 202. This correspond to an annulus whose outer and inner radii have a ratio of 198.0 , and thus to an outer radius that is virtually infinite. The results $\hat{\pi}_{h}^{l}=.5003, \hat{\pi}_{v}^{l}=.4990$, and $\hat{\pi}_{h v}^{l}=.3224$, are, as they should be, very close to those that appear in the first line of Table 3.2 and that are familiar from experiments on the square.

In spite of the difficulties created by the hole, it is nonetheless important, especially for $\S 3.7$, to estimate, by simulation and without recourse to a conformally equivalent cylinder, crossing probabilities in exterior domains. To do so we do not use extrapolation but take advantage in another way of conformal invariance. For example, if we have an annulus bounded by circles of radii $r_{1}$ and $r_{2}$ then we introduce a second independent disk of radius $r_{2}$ with independent probabilities for occupancy of the lattice points it contains, except at the boundary. To obtain an admissible path for a given configuration of open and closed sites we start from the inner interval $\alpha$, or as usual from a point in a band about $\alpha$, but when we arrive at an open site with a neighbor outside the larger disk, we open the corresponding site on the second disk, and then move as far as possible through it on open sites, allowing ourselves to return under the same conditions to the original annulus, and indeed to pass back and forth 


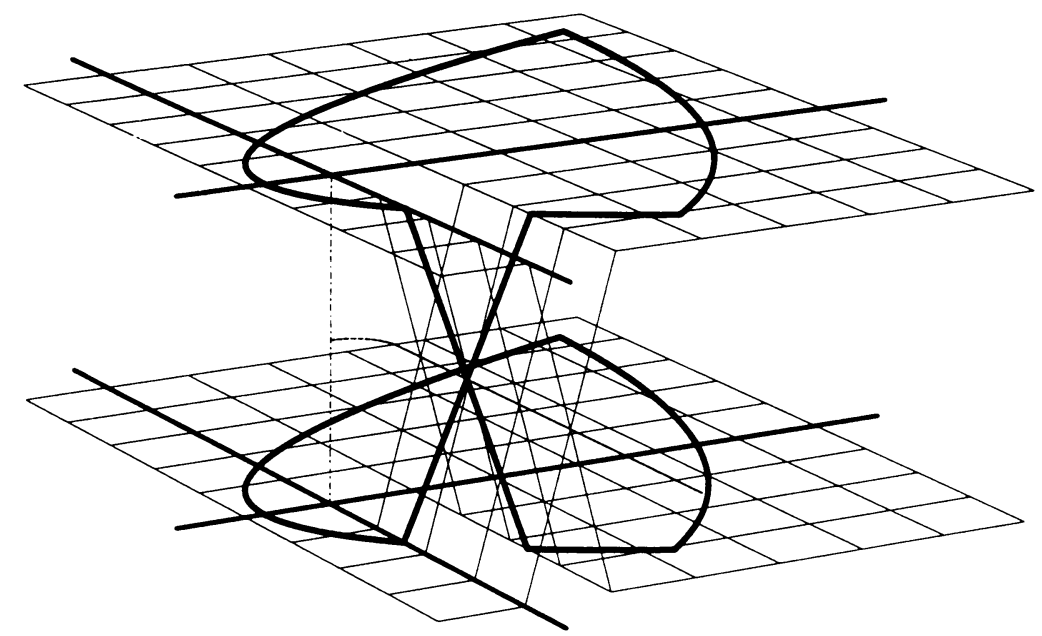

Figure 3.6a. A two-fold covering of the square lattice and the image of a parallelogram by the map $z \rightarrow z^{2}$.

between the annulus and the supplementary disk arbitrarily many times in the effort to reach the second interval $\beta$ on the inner boundary of the annulus.

Thus, in effect, we perform a roughly conformal glueing of the annulus and the disk in order to obtain on the Riemann sphere the exterior of the inner circle. With a sample of 100,000 configurations the probability $\pi_{h}$ for radii of 70 and 350 was estimated to 0.5078 and for radii of 100 and 600 it was found to be 0.5013 . These values can be regarded as encouraging confirmations of the technique, although the first is somewhat high, differing from .5 by more than our benchmark of .005 .

3.6. Branched percolation. If we apply the map $w=z^{2}$ to a region $D$ in the $z$-plane containing the origin, then this region is realized as a branched covering of a region $D^{\prime}$ in the $w$-plane. We can introduce crossing probabilities for $D$ in the usual way; we can also lift the percolating lattice from $D^{\prime}$ to $D$ and calculate crossing probabilities with respect to it. The most general form of conformal invariance implies that they are the same.

The lattice in $D$ is best viewed, as in Figure 3.6a as a two-fold covering of the lattice in $D^{\prime}$, each site in $D^{\prime}$, except those at the branch point, being covered by two sites. As the broken vertical line suggests, the points at the origin on the two sheets are to be identified. The image in the $w$-plane of the the parallelogram of aspect ratio 2.224 and interior angles $3 \pi / 8$ and $5 \pi / 8$ is shown in Figure 3.6b, in which the fine line is the branch cut. It is clear from the appearance of the parabolic arc, that the upper and lower sides of the parallelogram are horizontal. The left and right sides are therefore not vertical. The double covering of the same curve appears in Figure 3.6a. Horizontal crossings are from the sites marked by small squares on one sheet to those on the second sheet. Vertical crossings are from circular sites to circular sites. As indicated a site can be both circular and square. A site is taken to be square if it is joined to a neighbor by a bond that passes through the image of a horizontal side. The circular sites are introduced in a similar fashion.

Since the problem is not in principle affected by a shift of the lattice, one 


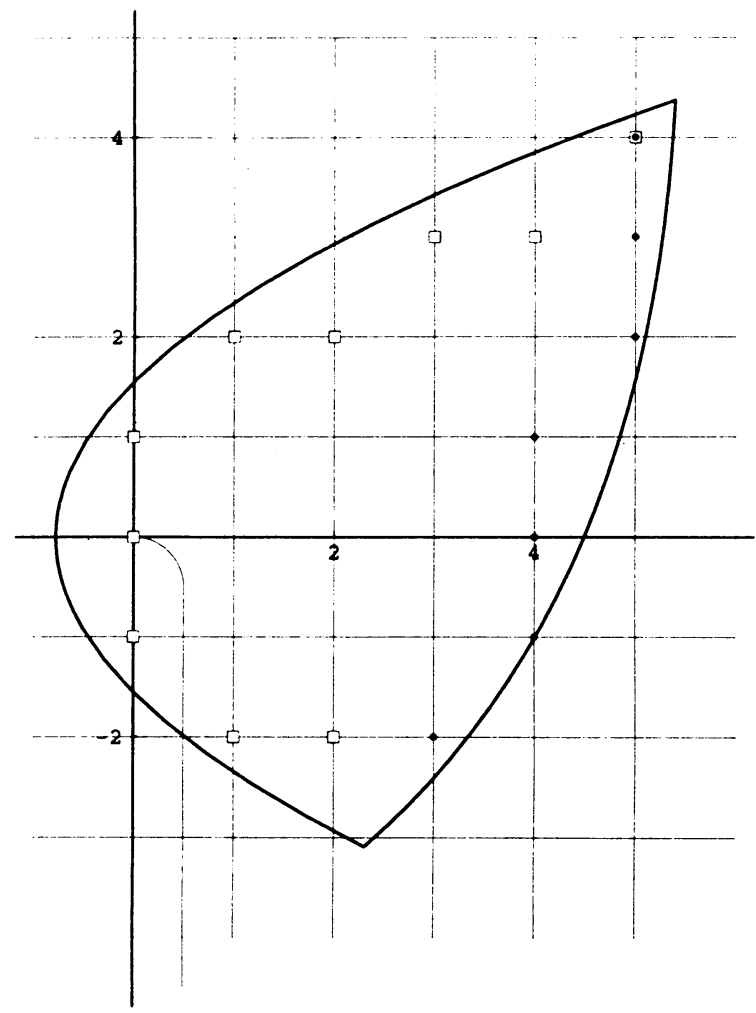

FIGURE 3.6b. The projection of the image of the parallelogram drawn in Figure 3.6a.

could suppose that the sites are never at the branch point. This is not, however, always wise. At a branch point the effect observed in the previous section is even more exaggerated since the number $\alpha$ that appears there is now 2 , so that an indeterminacy of .01 could be magnified to .1 . Nonetheless, to our astonishment, on choosing the square lattice such that the branch point is a site, which will then have eight neighbors rather than four, we obtained simulated values remarkably close to the true values, perhaps as a result of an implicit overcompensation. The difference between simulated and predicted values is rarely more than .002 . Other choices for the branch point, for which the data are not included, turned out to be far less felicitous.

The results, all for regions $D$ in the form of parallelograms, are presented in Table 3.6, which is self-explanatory. Experiments were performed for four values $\alpha$ of the interior angles of the parallelograms, and four values of the aspect ratio. The region $D^{\prime}$ always contained more than 200,000 sites, occasionally many more. In these experiments the second definition of the probabilities $\pi_{d}$ was used. (See §3.3.) The probabilities $\pi_{\bar{d}}$ are those for a crossing between the two intervals complementary to those defining $\pi_{d}$. Thus the sum of $\hat{\pi}_{d}$ and $\hat{\pi}_{d}$ is expected to be 1 by duality and universality. Observe that the extreme value for $\hat{\pi}_{h v}^{D}$ is larger than $\pi_{h}^{c f t}$ and thus is spurious. Of course this is not a weakness of the present experiment but a consequence of the experiment of $\S 3.2$ where the values of $\hat{\pi}_{h}$ and $\hat{\pi}_{h v}$ for extreme values of $r$ carry important statistical and systematic errors. 


\begin{tabular}{|c|c|c|c|c|}
\hline R & 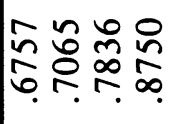 & 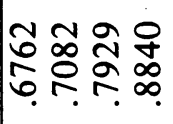 & 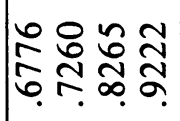 & 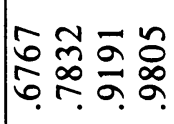 \\
\hline 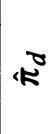 & ஸิ & 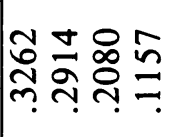 & 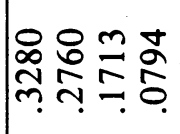 & 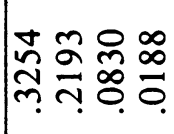 \\
\hline$\sum^{k}$ & 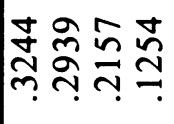 & 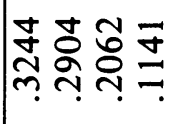 & 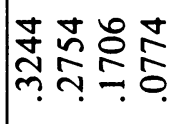 & 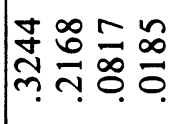 \\
\hline స్చే & 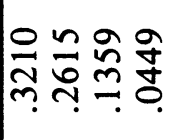 & 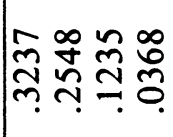 & 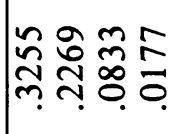 & 尔 \\
\hline 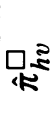 & 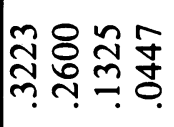 & 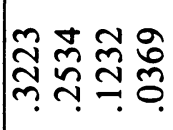 & 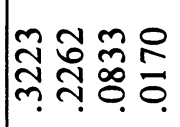 & 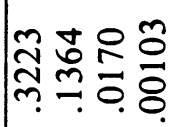 \\
\hline הN & 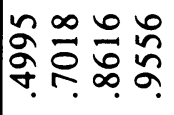 & 尽三 & 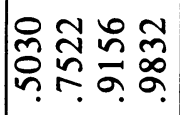 & 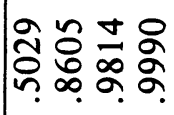 \\
\hline$\vec{R}$ & 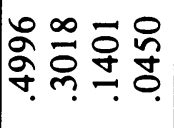 & $\begin{array}{l}\infty \\
\infty \\
0 \\
0\end{array}$ & 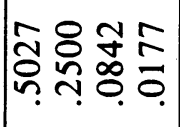 & 종 \\
\hline S & 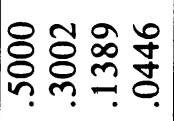 & 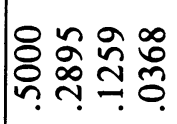 & 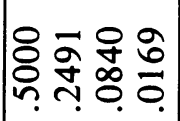 & 요 \\
\hline$\theta$ & $\int_{N}^{\frac{k}{N}} \frac{k}{N} \frac{k}{N}$ & 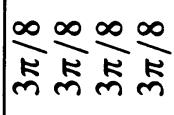 & 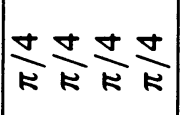 & $\int_{k}^{\infty} \frac{\infty}{k}{ }_{k}^{\infty} \frac{\infty}{k}$ \\
\hline- & 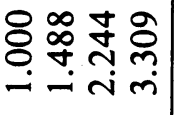 & 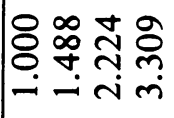 & 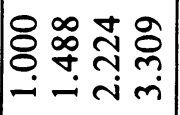 & 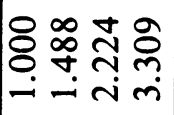 \\
\hline
\end{tabular}


3.7. Percolation on compact Riemann surfaces. Any compact Riemann surface $S$ can be realized as a branched covering of the projective line $\mathbb{P}$, thus of $\mathbb{C}$ with the point at infinity added. Combining the constructions for unbounded domains together with those for branched coverings, we can introduce percolation on the surface. Various crossing probabilities can be introduced. In particular each state $s$ yields a topological space, $X_{s}$, formed by all bonds and the open sites and imbedded in $S$, and thus a homomorphism $H_{1}\left(X_{S}\right) \rightarrow H_{1}(S)$ from the first homology group of $X_{s}$ to that of $S$. We can ask for the probability, always at criticality, that a given subgroup $Z$ of $H_{1}(S)$ is contained in the image, and expect that the response depends only on the conformal class of $S$.

We implicitly define percolation as being with respect to the model $M_{0}$ that defines the standard conformal structure on $\mathbb{P}$ and thus on $S$. Other choices of model and conformal structure would be possible. It is a matter of compatibility.

It is possible to define a Riemann surface otherwise than as a branched covering. For example, an elliptic curve can be obtained as the quotient of $\mathbb{C}$ by a lattice $L=\mathbb{Z}+\mathbb{Z} \omega$, and the percolation can be introduced directly on the surface as the percolation by sites on $a L^{\prime}$ (in the limit $a \rightarrow 0$ ) with $L^{\prime}=\mathbb{Z}+\mathbb{Z} / \sqrt{-1}$ and with (appropriately defined) periodic boundary conditions. Conformal invariance implies that the probabilities on the torus

$$
S_{1}=\mathbb{C} /(\mathbb{Z}+\mathbb{Z} \omega)
$$

and on the branched covering $S_{2}$ of the $x$-plane defined by

$$
y^{2}=\prod_{i=1}^{4}\left(x-\omega_{i}\right)
$$

are the same provided the two curves are isomorphic.

To define the percolation on (3.7a) we took $\omega=i$, and used a square lattice of mesh $\frac{1}{500}$. The elliptic curve $S_{2}$ will be conformally equivalent to $S_{1}$ if the points $\omega_{i}$ lie on the corners of a square. We took the lattice defining the percolation to be the usual square lattice of mesh 1 , and $\omega_{i}$ to be the four corners of a square with center 0 and sides of length 282 parallel to the two axes. The branch cuts were along the two horizontal lines and were treated as for branched percolation. The infinite parts of the lattices on the two sheets were handled as for exterior domains by means of a rough glueing along circles of radius 399 .

The elements of $H_{1}\left(S_{1}\right)$ are naturally labeled by pairs of integers $(m, n)$, and $(m, n)$ and $(-m,-n)$ generate the same subgroup. It is easy to persuade oneself that only primitive elements, those for which the greatest common divisor of $m$ and $n$ is 1 , appear as generators of subgroups $Z$ that occur with positive probability. We label such subgroups by a generator. Besides subgroups with one generator, the trivial subgroup 0 and the full subgroup $H=H_{1}$ may occur. 

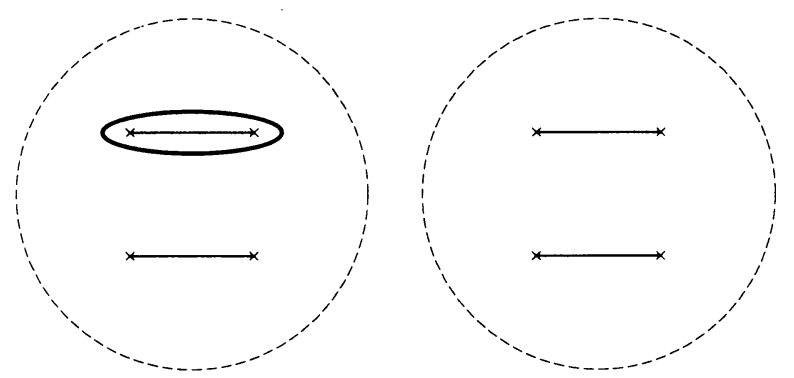

Figure 3.7a. A possible crossing on the elliptic curve $S_{2}$ for $\pi(0,1)$. (The "x"s are the branch points, the straight lines between them cuts; the dashed line indicates where the conformal glueing with another open disk takes place.)
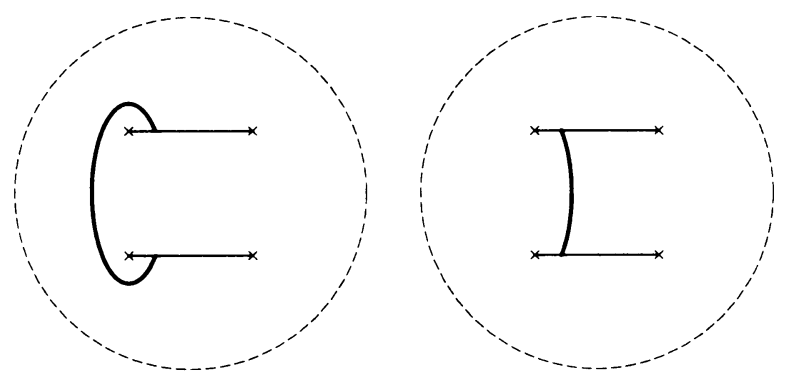

Figure 3.7b. A possible crossing on the elliptic curve $S_{2}$ for $\pi(1,0)$.

We choose a conformal equivalence of $S_{1}$ and $S_{2}$ that takes the loop around one of the branch cuts to the class $(0,1)$. Figure 3.7 a shows two open sets containing the branch points (crosses) on the upper and lower branch of the covering. The cuts have been chosen as indicated. The thick ellipse is one possible generators of the class $(0,1)$. Figure $3.7 \mathrm{~b}$ shows a generator for the class $(1,0)$. These choices fix, in particular, an isomorphism of the two homology groups that allows us to use the same labels for pertinent subgroups of $H_{1}\left(S_{1}\right)$ and $H_{1}\left(S_{2}\right)$. The results of our simulations are given in Table 3.7. Rather than measure the probability $\hat{\pi}(0)$ that no homology class other than 0 occurs in the image directly, we have for the purposes of the table simply defined it to 1 minus the sum of the probabilities measured. In addition to those given in the table we measured the probabilities for $(2, \pm 1),(1, \pm 2)$. Since the classes $(1, \pm 2)$ and $(2, \pm 1)$ (taken together) appear in only 26 of the 210000 configurations examined for $S_{1}$ and only 16 of the 107900 examined for $S_{2}$, this definition appeared admissible.

We observe that the probability for $H$ is substantially, but not intolerably, higher for $S_{2}$ than for $S_{1}$ and the probability for $\{0\}$ substantially lower. The probabilities for $S_{1}$ are presumably closer to the truth because they are nearly equal, as universality and duality demand. Moreover, simulations of $S_{2}$ required the use of two devices introduced earlier: conformal glueing of an open disk at infinity and branch points with 8 nearest neighbors instead of 4. As we saw, both artifices have limitations (See $\S 3.5$ and §3.6.) that could 
TABle 3.7. Probabilities of the first few subgroups of the homology group for the two elliptic curves $S_{1}$ and $S_{2}$.

\begin{tabular}{|c|c|c|c|c|c|c|}
\hline & $\hat{\pi}(H)$ & $\hat{\pi}(1,0)$ & $\hat{\pi}(0,1)$ & $\hat{\pi}(1,1)$ & $\hat{\pi}(1,-1)$ & $\hat{\pi}(0)$ \\
\hline$S_{1}$ & 0.3101 & 0.1693 & 0.1686 & 0.0205 & 0.0209 & 0.3106 \\
\hline$S_{2}$ & 0.3223 & 0.1700 & 0.1682 & 0.0206 & 0.0205 & 0.2983 \\
\hline
\end{tabular}

cause the discrepancy between the value of $\hat{\pi}(H)$ for $S_{1}$ and $S_{2}$, as well as the discrepancy of .024 between $\hat{\pi}(H)$ and $\hat{\pi}(0)$ that follows from it and our definitions. The values of the probabilities for subgroups of rank one are, however, quite close and well within the statistical errors.

\section{REFERENCES}

[AB] M. Aizenman and David J. Barsky, Sharpness of the phase transition in percolation models, Comm. Math. Phys. 108 (1987), 489-526.

[BH] S. R. Broadbent, J. H. Hammersley, Percolation processes, I. Crystals and mazes, Math. Proc. Cambridge Philos. Soc. 53 (1957), 629-641.

[BPZ] A. A. Belavin, A. M. Polyakov, and A. B. Zamolodchikov, Infinite conformal symmetry in two-dimensional quantum field theory, Nuclear Phys. B 241 (1984), 333-380.

[C1] John L. Cardy, Conformal invariance and surface critical behavior, Nuclear Phys. B 240 (1984), 514-522.

[C2] Effect of boundary conditions on the operator content of two-dimensional conformally invariant theories, Nuclear Phys. B 275 (1986), 200-218.

[C3] Boundary conditions, fusion rules and the Verlinde formula, Nuclear Phys. B 324 (1989), 581-596.

[C4] Critical percolation in finite geometries, J. Phys. A 25 (1992), L201.

[E1] John W. Essam, Graph theory and statistical physics, Discrete Math 1 (1971), 83-112.

[E2] _ Percolation theory, Rep. Progr. Phys. 43 (1980), 833-912.

[F1] M. E. Fisher, The theory of equilibrium critical phenomena, Rep. Progr. Phys. 30 (1967), 615-730.

[F2] Scaling, universality and renormalization group theory, Critical Phenomena (F. J. W. Hahne, ed.), Lecture Notes in Phys., vol. 186, Springer-Verlag, New York, 1983, pp. 1139.

[GJ] James Glimm and Arthur Jaffe, Quantum physics, Springer-Verlag, New York, 1981.

[G] G. Grimmett, Percolation, Springer-Verlag, New York, 1989.

[H] P. Heller, Experimental investigations of critical phenomena, Rep. Progr. Phys. 30 (1967), 731-826.

[K] H. Kesten, Percolation theory for mathematicians, Birkhäuser, Boston, 1982.

[U] R. P. Langlands, C. Pichet, P. Pouliot, and Y. Saint-Aubin, On the universality of crossing probabilities in two-dimensional percolation, J. Statist. Phys. 67 (1992), 553-574.

[L] R. P. Langlands, Dualität bei endlichen Modellen der Perkolation, Math. Nach. 160 (1993), 7-58.

[LL] R. P. Langlands and M.-A. Lafortune, Finite models for percolation, submitted for publication in the Corwin Memorial Volume, Contemp. Math., Amer. Math. Soc., Providence, RI.

[M] David S. McLachlan, Michael Blaszkiewicz, and Robert E. Newnham, Electrical resistivity of composites, J. Amer. Ceram. Soc. 73 (1990), 2187-2203.

[NF] D. R. Nelson and M. E. Fisher, Soluble renormalization groups and scaling fields for the low-dimensional Ising systems, Ann. Physics 91 (1975), 226-274.

[P] Jean Perrin, Les atomes, Coll. Idées, vol. 222, Gallimard, Paris, 1970. 
[RW1] Alvany Rocha-Caridi and Nolan Wallach, Characters of irreducible representations of the Lie algebra of vector fields on the circle, Invent. Math. 72 (1983), 57-75.

[RW2] Characters of irreducible representations of the Virasoro algebra, Math. Z. 185 (1984), 1-21.

[SA] Yvan Saint-Aubin, Phénomènes critiques en deux dimensions et invariance conforme, Course notes, Univ. of Montréal, 1987.

[S] Jan V. Sengers and Anneke Levelt Sengers, The critical region, Chemical and Engineering News, 10 June 1968, pp. 104-118.

[Wo] Po-zen Wong, The statistical physics of sedimentary rock, Physics Today 41 (1988), 24-32.

[W] F. Y. Wu, The Potts model, Rev. Modern Phys. 54 (1982), 235-268.

[Y] F. Yonezawa, S. Sakamoto, K. Aoki, S. Nosé, and M. Hori, Percolation in Penrose tiling and its dual-in comparison with analysis for Kagomé, dice and square lattices, J. NonCrys. Solids 106 (1988), 262-269.

[Z] Robert Ziff, On the spanning probability in 2D percolation, Phys. Rev. Lett. 69 (1992), 2670-2673.

School of Mathematics, Institute for Advanced Study, Princeton, New Jersey 08540

E-mail address: rplomath.ias.edu

Département de Physique and Centre de Recherches Mathematiques, Universite de Montréal, C.P. 6128A, Montreal, Québec, Canada H3C 3J7

Current address: Department of Physics, Rutgers University, New Brunswick, New Jersey 08855

E-mail address: pouliot@physics.rutgers.edu

Centre de Recherches Mathematiques and Departement de Mathematiques et de Statistique, Universite de Montréal, C.P. 6128A, Montréal, Quebec, Canada H3C 3J7

E-mail address: saint@crm.umontreal.ca 